\title{
Characteristics of Private Schools in the United States: Results From the 2011-12 Private School Universe Survey
}

First Look 



\section{Characteristics of Private Schools in the United States: Results From the 2011-12 Private School Universe Survey}

First Look

\section{JULY 2013}

Stephen P. Broughman

National Center for Education Statistics

Nancy L. Swaim

U.S. Census Bureau

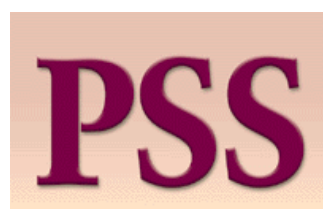

Private School Universe Survey 


\section{U.S. Department of Education}

Arne Duncan

Secretary

Institute of Education Sciences

John Q. Easton

Director

\section{National Center for Education Statistics}

Jack Buckley

Commissioner

\section{Elementary/Secondary and Library Studies Division}

Jeffrey Owings

Associate Commissioner

The National Center for Education Statistics (NCES) is the primary federal entity for collecting, analyzing, and reporting data related to education in the United States and other nations. It fulfills a congressional mandate to collect, collate, analyze, and report full and complete statistics on the condition of education in the United States; conduct and publish reports and specialized analyses of the meaning and significance of such statistics; assist state and local education agencies in improving their statistical systems; and review and report on education activities in foreign countries.

NCES activities are designed to address high-priority education data needs; provide consistent, reliable, complete, and accurate indicators of education status and trends; and report timely, useful, and high-quality data to the U.S. Department of Education, the Congress, the states, other education policymakers, practitioners, data users, and the general public. Unless specifically noted, all information contained herein is in the public domain.

We strive to make our products available in a variety of formats and in language that is appropriate to a variety of audiences. You, as our customer, are the best judge of our success in communicating information effectively. If you have any comments or suggestions about this or any other NCES product or report, we would like to hear from you. Please direct your comments to

NCES, IES, U.S. Department of Education

1990 K Street NW

Washington, DC 20006-5651

July 2013

The NCES Home Page address is http://nces.ed.gov.

The NCES Publications and Products address is http://nces.ed.gov/pubsearch.

This publication is only available online. To download, view, and print the report as a PDF file, go to the NCES Publications and Products address shown above.

\section{Suggested Citation}

Broughman, S.P., and Swaim, N.L. (2013). Characteristics of Private Schools in the United States: Results From the 2011-12 Private School Universe Survey (NCES 2013-316). U.S. Department of Education. Washington, DC: National Center for Education Statistics. Retrieved [date] from http://nces.ed.gov/pubsearch.

\section{Content Contact}

Stephen Broughman

(202) 502-7315

stephen.broughman@ed.gov 


\section{Contents}

\section{Page}

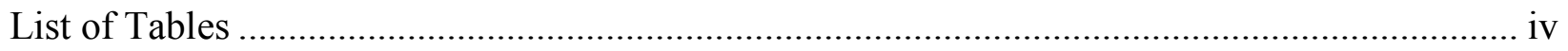

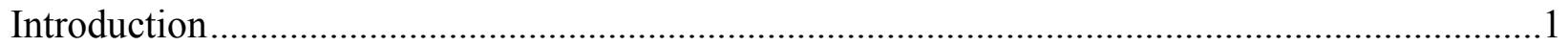

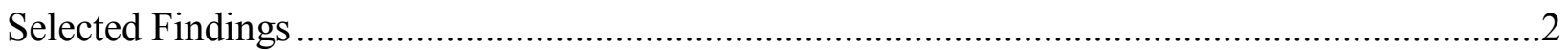

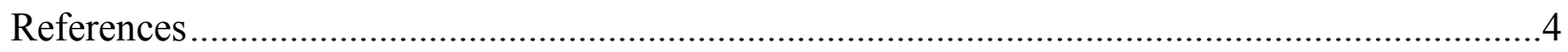

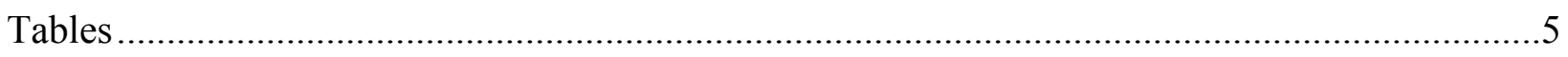

Appendix A - Glossary .................................................................................................... A-1

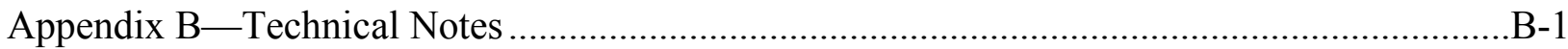

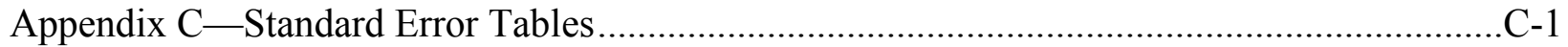




\section{List of Tables}

Table

Page

1 Number and percentage distribution of private schools, students, and full-time equivalent (FTE) teachers, by selected school characteristics: United States, 2011-12...

2 Number and percentage distribution of private schools, students, and full-time equivalent (FTE) teachers, by religious or nonsectarian orientation of school: United States, 2011-12...

3 Percentage distribution of private schools, by program emphasis and selected

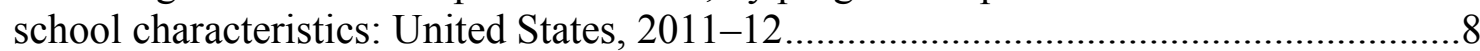

$4 \quad$ Number and percentage distribution of private schools, by urbanicity type and selected school characteristics: United States, 2011-12.

$5 \quad$ Number and percentage distribution of private school students, by urbanicity type and selected school characteristics: United States, 2011-12..

$6 \quad$ Number and percentage distribution of private school students, by grade and private school typology: United States, 2011-12

7 Average private school size, by school level and selected school characteristics: United States, 2011-12

$8 \quad$ Number and percentage distribution of private schools, by school size and selected school characteristics: United States, 2011-12.

$9 \quad$ Percentage distribution of private school students, by racial/ethnic background and selected school characteristics: United States, 2011-12.

10 Percentage male enrollment in private schools and percentage distribution of private schools, by coeducational category and selected school characteristics: United States, 2011-12

11 Number and percentage distribution of private school teachers (headcount), by work status and selected characteristics: United States, 2011-12

12 Pupil/teacher ratio of private schools, by school level and selected characteristics: United States, 2011-12

13 Percentage of private schools with 12th-graders, number of graduates, graduation rate, and percentage of graduates who attended 4-year colleges, by selected characteristics: United States, 2010-11

14 Number of private schools, students, and teachers (headcount), by school membership in private school associations: United States, 2011-12 ...

15 Number of private schools, students, full-time equivalent (FTE) teachers, and 2010-11 high school graduates, by state: United States, 2011-12. .20 


\section{List of Tables-Continued}

Table

Page

\section{Appendix B-Technical Notes Table}

B-1 Number of responding and nonresponding schools, out-of-scope cases, and school response rate, by frame: 2011-12

\section{Appendix C-Standard Error Tables}

C-1 Standard errors for number and percentage distribution of private schools, students, and full-time equivalent (FTE) teachers, by selected school characteristics: United States, 2011-12

C-2 Standard errors for number and percentage distribution of private schools, students, and full-time equivalent (FTE) teachers, by religious or nonsectarian orientation of school: United States, 2011-12.

C-3 Standard errors for percentage distribution of private schools, by program emphasis and selected school characteristics: United States, 2011-12

C-4 Standard errors for number and percentage distribution of private schools, by urbanicity type and selected school characteristics: United States, 2011-12.

C-5 Standard errors for number and percentage distribution of private school students, by urbanicity type and selected school characteristics: United States, 2011-12.

C-6 Standard errors for number and percentage distribution of private school students, by grade and private school typology: United States, 2011-12

C-7 Standard errors for average private school size, by school level and selected school characteristics: United States, 2011-12 .

C-8 Standard errors for number and percentage distribution of private schools, by school size and selected school characteristics: United States, 2011-12

C-9 Standard errors for percentage distribution of private school students, by racial/ethnic background and selected school characteristics: United States, 2011-12.

C-10 Standard errors for percentage male enrollment in private schools and percentage distribution of private schools, by coeducational category and selected school characteristics: United States, 2011-12.

C-11 Standard errors for number and percentage distribution of private school teachers (headcount), by work status and selected characteristics: United States, 2011-12

C-12 Standard errors for pupil/teacher ratio of private schools, by school level and selected characteristics: United States, 2011-12 


\section{List of Tables-Continued}

Table

Page

C-13 Standard errors for percentage of private schools with 12th-graders, number of graduates, graduation rate, and percentage of graduates who attended 4-year colleges, by selected characteristics: United States, 2010-11

C-14 Standard errors for number of private schools, students, and teachers (headcount), by school membership in private school associations: United States, 2011-12

C-15 Standard errors for number of private schools, students, full-time equivalent (FTE) teachers, and 2010-11 high school graduates, by state: United States, 2011-12 


\section{Introduction}

In 1988, the National Center for Education Statistics (NCES) developed a private school data collection that improved on the sporadic collection of private school data dating back to 1890 by developing an alternative to commercially available private school sampling frames. Since 1989, the U.S. Bureau of the Census has conducted the biennial Private School Universe Survey (PSS) for NCES. The PSS is designed to generate biennial data on the total number of private schools, students, and teachers, and to build a universe of private schools to serve as a sampling frame of private schools for NCES sample surveys. For more information about the methodology and design of the PSS, please see the Technical Notes in appendix B of this report.

The target population for the PSS is all schools in the 50 states and the District of Columbia that are not supported primarily by public funds, provide classroom instruction for one or more of grades kindergarten through 12 (or comparable ungraded levels), and have one or more teachers. Organizations or institutions that provide support for home schooling, but do not provide classroom instruction, are not included.

The 2011-12 PSS data were collected between September 2011 and May 2012. All data are for the 2011-12 school year except the high school graduate data, which are for the 2010-11 school year.

Because the purpose of this report is to introduce new NCES survey data through the presentation of tables containing descriptive information, only selected findings are listed below. These findings are purely descriptive in nature and are not meant to imply causality. These findings have been chosen to demonstrate the range of information available from the 2011-12 PSS rather than to discuss all of the observed differences, emphasize any particular issue, or make comparisons over time.

The tables in this report contain counts and percentages demonstrating bivariate relationships. All of the results have been weighted to reflect the sample design and to account for nonresponse and other adjustments. Comparisons drawn in the selected findings have been tested for statistical significance at the .05 level using Student's $t$ statistics to ensure that the differences are larger than those that might be expected due to sampling variation. No adjustments were made for multiple comparisons. Many of the variables examined are related to one another, and complex interactions and relationships have not been explored. Statistical Analysis Software (SAS 9.2) and SUDAAN (10.0) were used to compute the statistics for this report. 


\section{Selected Findings}

- In the fall of 2011, there were 30,861 private elementary and secondary schools with 4,494,845 students and 420,880 full-time equivalent (FTE) teachers in the United States (table 1).

- Sixty-eight percent of private schools, enrolling 80 percent of private school students and employing 72 percent of private school FTE teachers in 2011-12, had a religious orientation or purpose (table 2).

- Seventy percent of private schools in 2011-12 emphasized a regular elementary/secondary program, 8 percent emphasized a Montessori program, 2 percent emphasized a special program emphasis, 6 percent emphasized a special education program, 3 percent emphasized an alternative program, and 12 percent emphasized an early childhood program (table 3 ).

- In 2011-12 there were more private schools in suburban locations (10,911), compared to those in cities $(10,005)$, followed by those in rural areas $(7,045)$, and then by those in towns $(2,900)$ (tables 1 and 4$)$.

- More private school students in 2011-12 were enrolled in schools located in cities $(1,900,639)$, followed by those enrolled in suburban schools $(1,672,501)$, followed by those in rural areas $(620,862)$, and then by those in towns $(300,842)$ (tables 1 and 5).

- More private school students in 2011-12 were enrolled in kindergarten $(449,819)$ than in any other grade level (table 6).

- The average school size in 2011-12 was 146 students across all private schools. Private school size differed by instructional level. On average, elementary schools had 108 students, secondary schools had 283 students, and combined schools had 190 students (table 7).

- Forty-four percent of all private schools in 2011-12 enrolled fewer than 50 students (table 8).

- Seventy-one percent of private school students in 2011-12 were White; 10 percent were Hispanic or Latino, regardless of race; 9 percent were Black or African American; 5 percent were Asian; 3 percent were of two or more races, and less than 1 percent was American Indian or Alaska Native, or Native Hawaiian or other Pacific Islander, respectively (table 9).

- Ninety-six percent of all private schools in 2011-12 were coeducational, while 2 percent enrolled all girls and 2 percent enrolled all boys (table 10).

- Seventy-eight percent of private school teachers in 2011-12 were full-time teachers; 5 percent taught less than full time, but at least three-quarter time; 9 percent taught less than three-quarter time, but at least one-half time; 5 percent taught less than one-half time, but at least one-quarter time; and 3 percent taught less than one-quarter time (table 11).

- The average pupil/teacher ratio in 2011-12 was 10.7 across all private schools. The average pupil/teacher ratio was lower in combined schools (9.4) than in elementary schools (11.5) or secondary schools (11.6) (table 12).

- Ninety-eight percent of 12th-graders enrolled in private schools around October 1, 2010 graduated by the fall of 2011 (table 13).

- Of the 305,842 private high school graduates in 2010-11, some 64 percent attended 4-year colleges by the fall of 2011 (table 13). 
- In 2011-12, there were 10,212 private schools that did not report membership in any private school association (table 14).

- In 2011-12, there were 200,000 or more students enrolled in private schools in each of California, Florida, Illinois, New York, Pennsylvania, and Texas (table 15). 


\section{References}

Beller, N.D. (1984). Private Elementary and Secondary Education, 1983 Enrollment, Teachers, and Schools (Bulletin). U.S. Department of Education. Washington, DC: National Center for Education Statistics.

Wolter, K.M. (1985). Introduction to Variance Estimation. New York: Springer-Verlag. 
Tables 
Table 1. Number and percentage distribution of private schools, students, and full-time equivalent (FTE) teachers, by selected school characteristics: United States, 2011-12

\begin{tabular}{|c|c|c|c|c|c|c|}
\hline \multirow{2}{*}{$\begin{array}{l}\text { School } \\
\text { characteristic }\end{array}$} & \multicolumn{2}{|c|}{ Schools } & \multicolumn{2}{|c|}{ Students } & \multicolumn{2}{|c|}{ FTE teachers } \\
\hline & Number & Percent & Number & Percent & Number & Percent \\
\hline Total & 30,861 & 100.0 & $4,494,845$ & 100.0 & 420,880 & 100.0 \\
\hline \multicolumn{7}{|l|}{ Private school typology } \\
\hline Catholic & 6,873 & 22.3 & $1,928,388$ & 42.9 & 138,071 & 32.8 \\
\hline Parochial & 2,910 & 9.4 & 728,669 & 16.2 & 49,364 & 11.7 \\
\hline Diocesan & 2,922 & 9.5 & 830,813 & 18.5 & 57,958 & 13.8 \\
\hline Private & 1,041 & 3.4 & 368,906 & 8.2 & 30,749 & 7.3 \\
\hline Other religious & 14,214 & 46.1 & $1,676,649$ & 37.3 & 163,139 & 38.8 \\
\hline Conservative Christian & 4,574 & 14.8 & 630,557 & 14.0 & 59,545 & 14.1 \\
\hline Other affiliated & 3,060 & 9.9 & 486,746 & 10.8 & 49,220 & 11.7 \\
\hline Unaffiliated & 6,579 & 21.3 & 559,347 & 12.4 & 54,374 & 12.9 \\
\hline Nonsectarian & 9,775 & 31.7 & 889,807 & 19.8 & 119,669 & 28.4 \\
\hline Regular & 4,882 & 15.8 & 611,529 & 13.6 & 74,519 & 17.7 \\
\hline Special emphasis & 3,280 & 10.6 & 173,773 & 3.9 & 25,624 & 6.1 \\
\hline Special education & 1,613 & 5.2 & 104,505 & 2.3 & 19,526 & 4.6 \\
\hline \multicolumn{7}{|l|}{ School level } \\
\hline Elementary & 19,697 & 63.8 & $2,124,106$ & 47.3 & 184,134 & 43.7 \\
\hline Secondary & 2,677 & 8.7 & 757,448 & 16.9 & 65,178 & 15.5 \\
\hline Combined & 8,488 & 27.5 & $1,613,291$ & 35.9 & 171,568 & 40.8 \\
\hline \multicolumn{7}{|l|}{ Program emphasis } \\
\hline Regular elementary/ & & & & & & \\
\hline secondary & 21,486 & 69.6 & $4,103,932$ & 91.3 & 358,928 & 85.3 \\
\hline Montessori & 2,439 & 7.9 & 88,670 & 2.0 & 14,455 & 3.4 \\
\hline Special program emphasis & 676 & 2.2 & 84,365 & 1.9 & 10,454 & 2.5 \\
\hline Special education & 1,859 & 6.0 & 117,262 & 2.6 & 21,741 & 5.2 \\
\hline Vocational/technical & $\ddagger$ & $\ddagger$ & $\ddagger$ & $\ddagger$ & $\ddagger$ & $\ddagger$ \\
\hline Alternative & 813 & 2.6 & 44,419 & 1.0 & 6,537 & 1.6 \\
\hline Early childhood & 3,583 & 11.6 & 55,093 & 1.2 & 8,670 & 2.1 \\
\hline \multicolumn{7}{|l|}{ Size (number of students) } \\
\hline Less than 50 & 13,459 & 43.6 & 267,883 & 6.0 & 45,630 & 10.8 \\
\hline $50-149$ & 7,667 & 24.8 & 701,112 & 15.6 & 80,887 & 19.2 \\
\hline 150-299 & 5,488 & 17.8 & $1,165,283$ & 25.9 & 101,691 & 24.2 \\
\hline $300-499$ & 2,447 & 7.9 & 940,949 & 20.9 & 78,076 & 18.6 \\
\hline $500-749$ & 1,103 & 3.6 & 665,253 & 14.8 & 52,954 & 12.6 \\
\hline 750 or more & 698 & 2.3 & 754,365 & 16.8 & 61,642 & 14.6 \\
\hline \multicolumn{7}{|l|}{ Region } \\
\hline Northeast & 7,447 & 24.1 & $1,078,014$ & 24.0 & 109,631 & 26.0 \\
\hline Midwest & 7,963 & 25.8 & $1,099,838$ & 24.5 & 91,307 & 21.7 \\
\hline South & 9,203 & 29.8 & $1,469,996$ & 32.7 & 145,226 & 34.5 \\
\hline West & 6,249 & 20.2 & 846,997 & 18.8 & 74,716 & 17.8 \\
\hline \multicolumn{7}{|l|}{ Urbanicity type } \\
\hline City & 10,005 & 32.4 & $1,900,639$ & 42.3 & 173,395 & 41.2 \\
\hline Suburban & 10,911 & 35.4 & $1,672,501$ & 37.2 & 155,632 & 37.0 \\
\hline Town & 2,900 & 9.4 & 300,842 & 6.7 & 28,265 & 6.7 \\
\hline Rural & 7,045 & 22.8 & 620,862 & 13.8 & 63,587 & 15.1 \\
\hline
\end{tabular}

¥ Reporting standards not met. There are fewer than 15 sample cases.

NOTE: Detail may not sum to totals because of rounding or missing values in cells with too few sample cases. These estimates include private schools that provide instruction for one or more of grades kindergarten through 12 (or comparable ungraded levels).

SOURCE: U.S. Department of Education, National Center for Education Statistics, Private School Universe Survey (PSS), 2011-2012. 
Table 2. Number and percentage distribution of private schools, students, and full-time equivalent (FTE) teachers, by religious or nonsectarian orientation of school: United States, 2011-12

\begin{tabular}{|c|c|c|c|c|c|c|}
\hline \multirow{2}{*}{$\begin{array}{l}\text { Religious or } \\
\text { nonsectarian orientation }\end{array}$} & \multicolumn{2}{|c|}{ Schools } & \multicolumn{2}{|c|}{ Students } & \multicolumn{2}{|c|}{ FTE teachers } \\
\hline & Number & Percent & Number & Percent & Number & Percent \\
\hline Total & 30,861 & 100.0 & $4,494,845$ & 100.0 & 420,880 & 100.0 \\
\hline $\begin{array}{l}\text { Religious orientation } \\
\text { Roman Catholic }\end{array}$ & $\begin{array}{r}21,086 \\
6,873\end{array}$ & $\begin{array}{l}68.3 \\
22.3\end{array}$ & $\begin{array}{l}3,605,038 \\
1,928,388\end{array}$ & $\begin{array}{l}80.2 \\
42.9\end{array}$ & $\begin{array}{l}301,210 \\
138,071\end{array}$ & $\begin{array}{l}71.6 \\
32.8\end{array}$ \\
\hline African Methodist Episcopal & $\ddagger$ & $\ddagger$ & $\ddagger$ & $\ddagger$ & $\ddagger$ & $\ddagger$ \\
\hline Amish & 1,260 & 4.1 & 33,419 & 0.7 & 1,782 & 0.4 \\
\hline Assembly of God & 292 & 0.9 & 36,940 & 0.8 & 3,232 & 0.8 \\
\hline Baptist & 1,970 & 6.4 & 203,984 & 4.5 & 20,327 & 4.8 \\
\hline Brethren & 78 & 0.3 & 8,193 & 0.2 & 673 & 0.2 \\
\hline Calvinist & 113 & 0.4 & 24,155 & 0.5 & 1,866 & 0.4 \\
\hline Christian (unspecified) & 4,518 & 14.6 & 607,130 & 13.5 & 59,634 & 14.2 \\
\hline Church of Christ & 141 & 0.5 & 28,811 & 0.6 & 2,581 & 0.6 \\
\hline Church of God & 104 & 0.3 & 8,931 & 0.2 & 819 & 0.2 \\
\hline Church of God in Christ & 23 & 0.1 & 1,539 & \# & 148 & \# \\
\hline Church of the Nazarene & 63 & 0.2 & 5,198 & 0.1 & 480 & 0.1 \\
\hline Disciples of Christ & $\ddagger$ & $\ddagger$ & $\ddagger$ & $\ddagger$ & $\ddagger$ & $\ddagger$ \\
\hline Episcopal & 374 & 1.2 & 95,189 & 2.1 & 11,084 & 2.6 \\
\hline Friends & 87 & 0.3 & 19,085 & 0.4 & 2,441 & 0.6 \\
\hline Greek Orthodox & 36 & 0.1 & 4,253 & 0.1 & 535 & 0.1 \\
\hline Islamic & 229 & 0.7 & 32,478 & 0.7 & 3,866 & 0.9 \\
\hline Jewish & 954 & 3.1 & 245,425 & 5.5 & 25,689 & 6.1 \\
\hline Latter Day Saints & $\ddagger$ & $\ddagger$ & $\ddagger$ & $\ddagger$ & $\ddagger$ & $\ddagger$ \\
\hline Lutheran Church-Missouri Synod & 994 & 3.2 & 118,444 & 2.6 & 9,247 & 2.2 \\
\hline Evangelical Lutheran Church In America & 120 & 0.4 & 8,941 & 0.2 & 763 & 0.2 \\
\hline Wisconsin Evangelical Lutheran Synod & 336 & 1.1 & 29,349 & 0.7 & 2,236 & 0.5 \\
\hline Other Lutheran & 82 & 0.3 & 6,563 & 0.1 & 524 & 0.1 \\
\hline Mennonite & 736 & 2.4 & 32,834 & 0.7 & 3,167 & 0.8 \\
\hline Methodist & 240 & 0.8 & 17,603 & 0.4 & 1,945 & 0.5 \\
\hline Pentecostal & 295 & 1.0 & 13,937 & 0.3 & 1,564 & 0.4 \\
\hline Presbyterian & 224 & 0.7 & 33,230 & 0.7 & 3,259 & 0.8 \\
\hline Seventh-Day Adventist & 800 & 2.6 & 48,684 & 1.1 & 3,974 & 0.9 \\
\hline Other & 118 & 0.4 & 9,773 & 0.2 & 1,043 & 0.2 \\
\hline Nonsectarian & 9,775 & 31.7 & 889,807 & 19.8 & 119,669 & 28.4 \\
\hline
\end{tabular}

\# Rounds to zero.

$\ddagger$ Reporting standards not met. There are fewer than 15 sample cases.

NOTE: Detail may not sum to totals because of rounding or missing values in cells with too few sample cases.

These estimates include private schools that provide instruction for one or more of grades kindergarten through 12 (or comparable ungraded levels).

SOURCE: U.S. Department of Education, National Center for Education Statistics, Private School Universe Survey (PSS), 2011-2012. 
Table 3. Percentage distribution of private schools, by program emphasis and selected school characteristics: United States, 2011-12

\begin{tabular}{|c|c|c|c|c|c|c|c|}
\hline $\begin{array}{l}\text { School } \\
\text { characteristic }\end{array}$ & $\begin{array}{r}\text { Regular } \\
\text { elementaryl } \\
\text { secondary }\end{array}$ & Montessori & $\begin{array}{r}\text { Special } \\
\text { program } \\
\text { emphasis }\end{array}$ & $\begin{array}{r}\text { Special } \\
\text { education }\end{array}$ & $\begin{array}{r}\text { Vocational/ } \\
\text { technical } \\
\text { programs }\end{array}$ & Alternative & $\begin{array}{r}\text { Early } \\
\text { childhood }\end{array}$ \\
\hline Total & 69.6 & 7.9 & 2.2 & 6.0 & $\ddagger$ & 2.6 & 11.6 \\
\hline \multicolumn{8}{|l|}{ Private school typology } \\
\hline Catholic & 96.2 & 0.7 & 0.4 & 1.1 & $\ddagger$ & $\ddagger$ & 1.5 \\
\hline Parochial & 99.1 & $\ddagger$ & 0.0 & $\ddagger$ & $\ddagger$ & 0.0 & $\ddagger$ \\
\hline Diocesan & 97.5 & $\ddagger$ & $\ddagger$ & 1.1 & $\ddagger$ & $\ddagger$ & 0.8 \\
\hline Private & 84.1 & 3.4 & $\ddagger$ & 3.7 & $\ddagger$ & $\ddagger$ & 6.0 \\
\hline $\begin{array}{l}\text { Other religious } \\
\text { Conservative }\end{array}$ & 86.8 & 1.0 & 1.3 & 1.2 & $\ddagger$ & 1.6 & 8.0 \\
\hline Christian & 92.5 & $\ddagger$ & 1.1 & 0.6 & $\ddagger$ & 2.0 & 3.6 \\
\hline Other affiliated & 87.7 & 0.7 & 2.2 & 1.4 & $\ddagger$ & 0.9 & 7.1 \\
\hline Unaffiliated & 82.3 & 1.7 & 1.1 & 1.5 & $\ddagger$ & 1.7 & 11.6 \\
\hline Nonsectarian & 26.0 & 23.0 & 4.7 & 16.5 & $\ddagger$ & 5.8 & 23.9 \\
\hline Regular & 52.1 & $\dagger$ & $\dagger$ & $\dagger$ & $\ddagger$ & $\dagger$ & 47.9 \\
\hline Special emphasis & $\dagger$ & 68.4 & 14.1 & $\dagger$ & $\ddagger$ & 17.3 & $\dagger$ \\
\hline Special education & $\dagger$ & $\dagger$ & $\dagger$ & 100.0 & $\ddagger$ & $\dagger$ & $\dagger$ \\
\hline \multicolumn{8}{|l|}{ School level } \\
\hline Elementary & 65.6 & 11.7 & 1.9 & 1.5 & $\ddagger$ & 1.3 & 18.1 \\
\hline Secondary & 75.3 & $\ddagger$ & 3.1 & 11.3 & $\ddagger$ & 9.8 & $\dagger$ \\
\hline Combined & 77.1 & 1.5 & 2.7 & 14.9 & $\ddagger$ & 3.5 & 0.3 \\
\hline \multicolumn{8}{|c|}{ Size (number of students) } \\
\hline Less than 50 & 46.4 & 13.9 & 1.9 & 7.7 & $\ddagger$ & 4.1 & 26.0 \\
\hline $50-149$ & 78.2 & 6.2 & 3.2 & 8.7 & $\ddagger$ & 2.8 & 0.9 \\
\hline $150-299$ & 93.3 & 1.5 & 2.0 & 2.4 & $\ddagger$ & 0.6 & $\ddagger$ \\
\hline $300-499$ & 96.1 & $\ddagger$ & 2.3 & 0.8 & $\ddagger$ & $\ddagger$ & $\ddagger$ \\
\hline $500-749$ & 97.9 & 0.0 & $\ddagger$ & $\ddagger$ & $\ddagger$ & $\ddagger$ & $\ddagger$ \\
\hline 750 or more & 98.7 & 0.0 & $\ddagger$ & $\ddagger$ & $\ddagger$ & $\ddagger$ & $\ddagger$ \\
\hline \multicolumn{8}{|l|}{ Region } \\
\hline Northeast & 62.6 & 6.1 & 2.2 & 10.1 & $\ddagger$ & 2.3 & 16.8 \\
\hline Midwest & 81.3 & 6.5 & 1.1 & 3.0 & $\ddagger$ & 1.3 & 6.7 \\
\hline South & 70.9 & 8.1 & 2.2 & 5.7 & $\ddagger$ & 3.1 & 10.0 \\
\hline West & 61.2 & 11.6 & 3.5 & 5.5 & $\ddagger$ & 4.1 & 14.1 \\
\hline \multicolumn{8}{|l|}{ Urbanicity type } \\
\hline City & 68.6 & 8.5 & 2.9 & 6.7 & $\ddagger$ & 2.4 & 10.8 \\
\hline Suburban & 59.1 & 10.9 & 2.0 & 7.6 & $\ddagger$ & 2.4 & 18.0 \\
\hline Town & 83.9 & 5.0 & 1.3 & 2.2 & $\ddagger$ & 1.9 & 5.7 \\
\hline Rural & 81.5 & 3.6 & 1.9 & 4.2 & $\ddagger$ & 3.6 & 5.2 \\
\hline
\end{tabular}

† Not applicable.

$\ddagger$ Reporting standards not met. There are fewer than 15 sample cases.

NOTE: Detail may not sum to totals because of rounding or missing values in cells with too few sample cases. These estimates include private schools that provide instruction for one or more of grades kindergarten through 12 (or comparable ungraded levels).

SOURCE: U.S. Department of Education, National Center for Education Statistics, Private School Universe Survey (PSS), 2011-2012. 
Table 4. Number and percentage distribution of private schools, by urbanicity type and selected school characteristics: United States, 2011-12

\begin{tabular}{|c|c|c|c|c|c|c|c|c|c|c|}
\hline \multirow{2}{*}{$\begin{array}{l}\text { School } \\
\text { characteristic }\end{array}$} & \multicolumn{2}{|c|}{ Total } & \multicolumn{2}{|c|}{ City } & \multicolumn{2}{|c|}{ Suburban } & \multicolumn{2}{|c|}{ Town } & \multicolumn{2}{|c|}{ Rural } \\
\hline & Number & Percent & Number & Percent & Number & Percent & Number & Percent & Number & Percent \\
\hline & 9519.9 & 32.83 & 8277 & 28.55 & 6202 & 21.39 & & & & \\
\hline Total & 30,861 & 100.0 & 10,005 & 100.0 & 10,911 & 100.0 & 2,900 & 100.0 & 7,045 & 100.0 \\
\hline \multicolumn{11}{|l|}{ Private school typology } \\
\hline Catholic & 6,873 & 22.3 & 2,833 & 28.3 & 2,492 & 22.8 & 933 & 32.2 & 614 & 8.7 \\
\hline Parochial & 2,910 & 9.4 & 1,107 & 11.1 & 1,089 & 10.0 & 463 & 16.0 & 251 & 3.6 \\
\hline Diocesan & 2,922 & 9.5 & 1,235 & 12.3 & 1,016 & 9.3 & 425 & 14.7 & 245 & 3.5 \\
\hline Private & 1,041 & 3.4 & 491 & 4.9 & 387 & 3.5 & 45 & 1.6 & 118 & 1.7 \\
\hline Other religious & 14,214 & 46.1 & 3,826 & 38.2 & 3,958 & 36.3 & 1,534 & 52.9 & 4,896 & 69.5 \\
\hline Conservative Christian & 4,574 & 14.8 & 1,133 & 11.3 & 1,443 & 13.2 & 673 & 23.2 & 1,326 & 18.8 \\
\hline Other affiliated & 3,060 & 9.9 & 1,120 & 11.2 & 1,060 & 9.7 & 277 & 9.6 & 603 & 8.6 \\
\hline Unaffiliated & 6,579 & 21.3 & 1,573 & 15.7 & 1,455 & 13.3 & 584 & 20.1 & 2,967 & 42.1 \\
\hline Nonsectarian & 9,775 & 31.7 & 3,346 & 33.4 & 4,461 & 40.9 & 433 & 14.9 & 1,535 & 21.8 \\
\hline Regular & 4,882 & 15.8 & 1,620 & 16.2 & 2,300 & 21.1 & 187 & 6.4 & 775 & 11.0 \\
\hline Special emphasis & 3,280 & 10.6 & 1,132 & 11.3 & 1,455 & 13.3 & 193 & 6.6 & 500 & 7.1 \\
\hline Special education & 1,613 & 5.2 & 593 & 5.9 & 706 & 6.5 & 53 & 1.8 & 260 & 3.7 \\
\hline \multicolumn{11}{|l|}{ School level } \\
\hline Elementary & 19,697 & 63.8 & 6,583 & 65.8 & 7,470 & 68.5 & 1,735 & 59.8 & 3,908 & 55.5 \\
\hline Secondary & 2,677 & 8.7 & 1,096 & 11.0 & 868 & 8.0 & 174 & 6.0 & 538 & 7.6 \\
\hline Combined & 8,488 & 27.5 & 2,326 & 23.2 & 2,572 & 23.6 & 991 & 34.2 & 2,599 & 36.9 \\
\hline \multicolumn{11}{|l|}{ Program emphasis } \\
\hline \multicolumn{11}{|l|}{ Regular elementary/ } \\
\hline secondary & 21,486 & 69.6 & 6,865 & 68.6 & 6,444 & 59.1 & 2,434 & 83.9 & 5,743 & 81.5 \\
\hline Montessori & 2,439 & 7.9 & 846 & 8.5 & 1,194 & 10.9 & 145 & 5.0 & 254 & 3.6 \\
\hline Special program & & & & & & & & & & \\
\hline emphasis & 676 & 2.2 & 285 & 2.9 & 220 & 2.0 & 37 & 1.3 & 135 & 1.9 \\
\hline Special education & 1,859 & 6.0 & 675 & 6.7 & 826 & 7.6 & 63 & 2.2 & 295 & 4.2 \\
\hline Vocational/technical & $\ddagger$ & $\ddagger$ & $\ddagger$ & $\ddagger$ & $\ddagger$ & $\ddagger$ & $\ddagger$ & $\ddagger$ & $\ddagger$ & $\ddagger$ \\
\hline Alternative & 813 & 2.6 & 244 & 2.4 & 259 & 2.4 & 56 & 1.9 & 254 & 3.6 \\
\hline Early childhood & 3,583 & 11.6 & 1,085 & 10.8 & 1,968 & 18.0 & 165 & 5.7 & 364 & 5.2 \\
\hline \multicolumn{11}{|l|}{ Size (number of students) } \\
\hline Less than 50 & 13,459 & 43.6 & 3,399 & 34.0 & 4,736 & 43.4 & 1,147 & 39.5 & 4,177 & 59.3 \\
\hline $50-149$ & 7,667 & 24.8 & 2,419 & 24.2 & 2,414 & 22.1 & 1,110 & 38.3 & 1,724 & 24.5 \\
\hline 150-299 & 5,488 & 17.8 & 2,260 & 22.6 & 2,037 & 18.7 & 464 & 16.0 & 727 & 10.3 \\
\hline $300-499$ & 2,447 & 7.9 & 1,041 & 10.4 & 1,015 & 9.3 & 138 & 4.7 & 253 & 3.6 \\
\hline $500-749$ & 1,103 & 3.6 & 511 & 5.1 & 458 & 4.2 & 34 & 1.2 & 100 & 1.4 \\
\hline 750 or more & 698 & 2.3 & 375 & 3.7 & 251 & 2.3 & $\ddagger$ & $\ddagger$ & 65 & 0.9 \\
\hline \multicolumn{11}{|l|}{ Region } \\
\hline Northeast & 7,447 & 24.1 & 1,855 & 18.5 & 3,522 & 32.3 & 354 & 12.2 & 1,716 & 24.4 \\
\hline Midwest & 7,963 & 25.8 & 2,084 & 20.8 & 2,196 & 20.1 & 1,184 & 40.8 & 2,498 & 35.5 \\
\hline South & 9,203 & 29.8 & 3,257 & 32.6 & 3,022 & 27.7 & 839 & 28.9 & 2,085 & 29.6 \\
\hline West & 6,249 & 20.2 & 2,809 & 28.1 & 2,170 & 19.9 & 523 & 18.0 & 747 & 10.6 \\
\hline
\end{tabular}

$\ddagger$ Reporting standards not met. There are fewer than 15 sample cases.

NOTE: Detail may not sum to totals because of rounding or missing values in cells with too few sample cases. These estimates include private schools that provide instruction for one or more of grades kindergarten through 12 (or comparable ungraded levels).

SOURCE: U.S. Department of Education, National Center for Education Statistics, Private School Universe Survey (PSS), $2011-2012$. 
Table 5. Number and percentage distribution of private school students, by urbanicity type and selected school characteristics: United States, 2011-12

\begin{tabular}{|c|c|c|c|c|c|c|c|c|c|c|}
\hline \multirow{2}{*}{$\begin{array}{l}\text { School } \\
\text { characteristic }\end{array}$} & \multicolumn{2}{|c|}{ Total } & \multicolumn{2}{|c|}{ City } & \multicolumn{2}{|c|}{ Suburban } & \multicolumn{2}{|c|}{ Town } & \multicolumn{2}{|c|}{ Rural } \\
\hline & Number & Percent & Number & Percent & Number & Percent & Number & Percent & Number & Percent \\
\hline Total & $4,494,845$ & 100.0 & $1,900,639$ & 100.0 & $1,672,501$ & 100.0 & 300,842 & 100.0 & 620,862 & 100.0 \\
\hline \multicolumn{11}{|l|}{ Private school typology } \\
\hline Catholic & $1,928,388$ & 42.9 & 902,991 & 47.5 & 763,520 & 45.7 & 155,088 & 51.6 & 106,790 & 17.2 \\
\hline Parochial & 728,669 & 16.2 & 306,755 & 16.1 & 308,215 & 18.4 & 74,708 & 24.8 & 38,991 & 6.3 \\
\hline Diocesan & 830,813 & 18.5 & 394,447 & 20.8 & 315,826 & 18.9 & 73,740 & 24.5 & 46,800 & 7.5 \\
\hline Private & 368,906 & 8.2 & 201,789 & 10.6 & 139,479 & 8.3 & 6,639 & 2.2 & 20,999 & 3.4 \\
\hline Other religious & $1,676,649$ & 37.3 & 620,859 & 32.7 & 581,312 & 34.8 & 113,854 & 37.8 & 360,625 & 58.1 \\
\hline Conservative Christian & 630,557 & 14.0 & 195,115 & 10.3 & 238,667 & 14.3 & 49,897 & 16.6 & 146,877 & 23.7 \\
\hline Other affiliated & 486,746 & 10.8 & 210,589 & 11.1 & 190,022 & 11.4 & 25,102 & 8.3 & 61,032 & 9.8 \\
\hline Unaffiliated & 559,347 & 12.4 & 215,154 & 11.3 & 152,623 & 9.1 & 38,854 & 12.9 & 152,716 & 24.6 \\
\hline Nonsectarian & 889,807 & 19.8 & 376,790 & 19.8 & 327,669 & 19.6 & 31,901 & 10.6 & 153,448 & 24.7 \\
\hline Regular & 611,529 & 13.6 & 267,682 & 14.1 & 209,978 & 12.6 & 22,753 & 7.6 & 111,116 & 17.9 \\
\hline Special emphasis & 173,773 & 3.9 & 67,403 & 3.5 & 71,294 & 4.3 & 7,182 & 2.4 & 27,894 & 4.5 \\
\hline Special education & 104,505 & 2.3 & 41,706 & 2.2 & 46,396 & 2.8 & 1,965 & 0.7 & 14,438 & 2.3 \\
\hline \multicolumn{11}{|l|}{ School level } \\
\hline Elementary & $2,124,106$ & 47.3 & 892,029 & 46.9 & 874,546 & 52.3 & 158,287 & 52.6 & 199,244 & 32.1 \\
\hline Secondary & 757,448 & 16.9 & 389,023 & 20.5 & 264,184 & 15.8 & 27,282 & 9.1 & 76,958 & 12.4 \\
\hline Combined & $1,613,291$ & 35.9 & 619,587 & 32.6 & 533,771 & 31.9 & 115,273 & 38.3 & 344,661 & 55.5 \\
\hline \multicolumn{11}{|l|}{ Program emphasis } \\
\hline \multicolumn{11}{|l|}{ Regular elementary/ } \\
\hline secondary & $4,103,932$ & 91.3 & $1,751,852$ & 92.2 & $1,502,206$ & 89.8 & 287,502 & 95.6 & 562,372 & 90.6 \\
\hline $\begin{array}{l}\text { Montessori } \\
\text { Special program }\end{array}$ & 88,670 & 2.0 & 34,211 & 1.8 & 41,613 & 2.5 & 3,404 & 1.1 & 9,443 & 1.5 \\
\hline emphasis & 84,365 & 1.9 & 36,089 & 1.9 & 30,542 & 1.8 & 3,237 & 1.1 & 14,497 & 2.3 \\
\hline Special education & 117,262 & 2.6 & 47,014 & 2.5 & 52,690 & 3.2 & 2,301 & 0.8 & 15,258 & 2.5 \\
\hline Vocational/technical & $\ddagger$ & $\ddagger$ & $\ddagger$ & $\ddagger$ & $\ddagger$ & $\ddagger$ & $\ddagger$ & $\ddagger$ & $\ddagger$ & $\ddagger$ \\
\hline Alternative & 44,419 & 1.0 & 14,848 & 0.8 & 16,395 & 1.0 & 2,206 & 0.7 & 10,970 & 1.8 \\
\hline Early childhood & 55,093 & 1.2 & 16,088 & 0.8 & 29,056 & 1.7 & 2,192 & 0.7 & 7,757 & 1.2 \\
\hline \multicolumn{11}{|l|}{ Size (number of students) } \\
\hline Less than 50 & 267,883 & 6.0 & 67,229 & 3.5 & 87,018 & 5.2 & 23,992 & 8.0 & 89,644 & 14.4 \\
\hline $50-149$ & 701,112 & 15.6 & 226,588 & 11.9 & 224,402 & 13.4 & 101,860 & 33.9 & 148,261 & 23.9 \\
\hline $150-299$ & $1,165,283$ & 25.9 & 482,357 & 25.4 & 432,984 & 25.9 & 96,058 & 31.9 & 153,885 & 24.8 \\
\hline $300-499$ & 940,949 & 20.9 & 403,564 & 21.2 & 389,154 & 23.3 & 51,974 & 17.3 & 96,257 & 15.5 \\
\hline $500-749$ & 665,253 & 14.8 & 310,220 & 16.3 & 274,733 & 16.4 & 20,075 & 6.7 & 60,225 & 9.7 \\
\hline 750 or more & 754,365 & 16.8 & 410,681 & 21.6 & 264,210 & 15.8 & $\ddagger$ & $\ddagger$ & 72,590 & 11.7 \\
\hline \multicolumn{11}{|l|}{ Region } \\
\hline Northeast & $1,078,014$ & 24.0 & 396,813 & 20.9 & 513,328 & 30.7 & 32,304 & 10.7 & 135,569 & 21.8 \\
\hline Midwest & $1,099,838$ & 24.5 & 415,977 & 21.9 & 396,843 & 23.7 & 135,739 & 45.1 & 151,279 & 24.4 \\
\hline South & $1,469,996$ & 32.7 & 630,251 & 33.2 & 479,677 & 28.7 & 95,140 & 31.6 & 264,928 & 42.7 \\
\hline West & 846,997 & 18.8 & 457,598 & 24.1 & 282,652 & 16.9 & 37,660 & 12.5 & 69,086 & 11.1 \\
\hline
\end{tabular}

$\ddagger$ Reporting standards not met. There are fewer than 15 sample cases.

NOTE: Detail may not sum to totals because of rounding or missing values in cells with too few sample cases. These estimates include private schools that provide instruction for one or more of grades kindergarten through 12 (or comparable ungraded levels).

SOURCE: U.S. Department of Education, National Center for Education Statistics, Private School Universe Survey (PSS), $2011-2012$. 
Table 6. Number and percentage distribution of private school students, by grade and private school typology: United States, 2011-12

\begin{tabular}{|c|c|c|c|c|c|c|c|c|c|c|}
\hline \multirow[b]{2}{*}{ Private school typology } & \multicolumn{2}{|c|}{ Kindergarten ${ }^{1}$} & \multicolumn{2}{|c|}{ First grade } & \multicolumn{2}{|c|}{ Second grade } & \multicolumn{2}{|c|}{ Third grade } & \multicolumn{2}{|c|}{ Fourth grade } \\
\hline & Number & Percent & Number & Percent & Number & Percent & Number & Percent & Number & Percent \\
\hline Total & 449,819 & 100.0 & 348,731 & 100.0 & 340,234 & 100.0 & 336,148 & 100.0 & 328,950 & 100.0 \\
\hline Catholic & 150,295 & 33.4 & 143,547 & 41.2 & 144,905 & 42.6 & 145,330 & 43.2 & 143,859 & 43.7 \\
\hline Parochial & 75,788 & 16.8 & 72,980 & 20.9 & 74,190 & 21.8 & 74,278 & 22.1 & 73,479 & 22.3 \\
\hline Diocesan & 64,433 & 14.3 & 61,853 & 17.7 & 62,038 & 18.2 & 62,172 & 18.5 & 61,613 & 18.7 \\
\hline Private & 10,074 & 2.2 & 8,713 & 2.5 & 8,677 & 2.6 & 8,880 & 2.6 & 8,766 & 2.7 \\
\hline $\begin{array}{l}\text { Other religious } \\
\text { Conservative }\end{array}$ & 182,306 & 40.5 & 146,368 & 42.0 & 139,696 & 41.1 & 136,513 & 40.6 & 132,819 & 40.4 \\
\hline Christian & 65,399 & 14.5 & 53,203 & 15.3 & 51,203 & 15.0 & 50,382 & 15.0 & 48,626 & 14.8 \\
\hline Other affiliated & 48,758 & 10.8 & 41,806 & 12.0 & 40,577 & 11.9 & 39,982 & 11.9 & 39,003 & 11.9 \\
\hline Unaffiliated & 68,149 & 15.2 & 51,359 & 14.7 & 47,916 & 14.1 & 46,149 & 13.7 & 45,190 & 13.7 \\
\hline Nonsectarian & 117,218 & 26.1 & 58,816 & 16.9 & 55,634 & 16.4 & 54,305 & 16.2 & 52,272 & 15.9 \\
\hline Regular & 76,350 & 17.0 & 39,560 & 11.3 & 38,361 & 11.3 & 37,622 & 11.2 & 36,994 & 11.2 \\
\hline Special emphasis & 39,271 & 8.7 & 17,521 & 5.0 & 14,855 & 4.4 & 13,379 & 4.0 & 11,077 & 3.4 \\
\hline \multirow[t]{2}{*}{ Special education } & 1,597 & 0.4 & 1,735 & 0.5 & 2,418 & 0.7 & 3,304 & 1.0 & 4,201 & 1.3 \\
\hline & \multicolumn{2}{|c|}{ Fifth grade } & \multicolumn{2}{|c|}{ Sixth grade } & \multicolumn{2}{|c|}{ Seventh grade } & \multicolumn{2}{|c|}{ Eighth grade } & \multicolumn{2}{|c|}{ Ninth grade } \\
\hline Private school typology & Number & Percent & Number & Percent & Number & Percent & Number & Percent & Number & Percent \\
\hline Total & 330,393 & 100.0 & 341,690 & 100.0 & 336,774 & 100.0 & 336,672 & 100.0 & 329,600 & 100.0 \\
\hline Catholic & 145,608 & 44.1 & 150,067 & 43.9 & 147,092 & 43.7 & 147,694 & 43.9 & 156,419 & 47.5 \\
\hline $\begin{array}{l}\text { Parochial } \\
\text { Diocesan } \\
\text { Private }\end{array}$ & $\begin{array}{r}74,471 \\
61,797 \\
9,340\end{array}$ & $\begin{array}{r}22.5 \\
18.7 \\
2.8\end{array}$ & $\begin{array}{l}74,539 \\
63,246 \\
12,282\end{array}$ & $\begin{array}{r}21.8 \\
18.5 \\
3.6\end{array}$ & $\begin{array}{l}70,597 \\
61,763 \\
14,732\end{array}$ & $\begin{array}{r}21.0 \\
18.3 \\
4.4\end{array}$ & $\begin{array}{l}70,095 \\
61,445 \\
16,154\end{array}$ & $\begin{array}{r}20.8 \\
18.3 \\
4.8\end{array}$ & $\begin{array}{l}17,405 \\
69,097 \\
69,917\end{array}$ & $\begin{array}{r}5.3 \\
21.0 \\
21.2\end{array}$ \\
\hline $\begin{array}{r}\text { Other religious } \\
\text { Conservative }\end{array}$ & 131,549 & 39.8 & 133,751 & 39.1 & 130,524 & 38.8 & 127,936 & 38.0 & 106,844 & 32.4 \\
\hline Christian & 48,965 & 14.8 & 50,480 & 14.8 & 49,956 & 14.8 & 48,526 & 14.4 & 42,617 & 12.9 \\
\hline Other affiliated & 38,413 & 11.6 & 39,069 & 11.4 & 37,666 & 11.2 & 37,683 & 11.2 & 31,019 & 9.4 \\
\hline Unaffiliated & 44,171 & 13.4 & 44,202 & 12.9 & 42,902 & 12.7 & 41,727 & 12.4 & 33,208 & 10.1 \\
\hline Nonsectarian & 53,236 & 16.1 & 57,872 & 16.9 & 59,158 & 17.6 & 61,042 & 18.1 & 66,338 & 20.1 \\
\hline Regular & 37,573 & 11.4 & 42,449 & 12.4 & 44,292 & 13.2 & 45,083 & 13.4 & 50,250 & 15.2 \\
\hline Special emphasis & 10,630 & 3.2 & 9,296 & 2.7 & 8,028 & 2.4 & 8,160 & 2.4 & 6,925 & 2.1 \\
\hline Special education & 5,033 & 1.5 & 6,127 & 1.8 & 6,838 & 2.0 & 7,799 & 2.3 & 9,163 & 2.8 \\
\hline
\end{tabular}

\begin{tabular}{|c|c|c|c|c|c|c|c|c|}
\hline \multirow[b]{2}{*}{ Private school typology } & \multicolumn{2}{|c|}{ Tenth grade } & \multicolumn{2}{|c|}{ Eleventh grade } & \multicolumn{2}{|c|}{ Twelfth grade } & \multicolumn{2}{|c|}{ Ungraded } \\
\hline & Number & Percent & Number & Percent & Number & Percent & Number & Percent \\
\hline Total & 324,538 & 100.0 & 318,313 & 100.0 & 314,503 & 100.0 & 58,479 & 100.0 \\
\hline Catholic & 152,561 & 47.0 & 148,381 & 46.6 & 148,592 & 47.2 & 4,040 & 6.9 \\
\hline $\begin{array}{l}\text { Parochial } \\
\text { Diocesan } \\
\text { Private }\end{array}$ & $\begin{array}{l}16,764 \\
67,793 \\
68,004\end{array}$ & $\begin{array}{r}5.2 \\
20.9 \\
21.0\end{array}$ & $\begin{array}{l}16,494 \\
65,996 \\
65,891\end{array}$ & $\begin{array}{r}5.2 \\
20.7 \\
20.7\end{array}$ & $\begin{array}{l}16,633 \\
65,825 \\
66,134\end{array}$ & $\begin{array}{r}5.3 \\
20.9 \\
21.0\end{array}$ & $\begin{array}{r}957 \\
1,740 \\
1,343\end{array}$ & $\begin{array}{l}1.6 \\
3.0 \\
2.3\end{array}$ \\
\hline $\begin{array}{l}\text { Other religious } \\
\text { Conservative }\end{array}$ & 103,820 & 32.0 & 99,835 & 31.4 & 96,817 & 30.8 & 7,872 & 13.5 \\
\hline $\begin{array}{c}\text { Christian } \\
\text { Other affiliated }\end{array}$ & $\begin{array}{l}41,505 \\
30,771\end{array}$ & $\begin{array}{r}12.8 \\
9.5\end{array}$ & $\begin{array}{l}39,900 \\
30,001\end{array}$ & $\begin{array}{r}12.5 \\
9.4\end{array}$ & $\begin{array}{l}38,062 \\
29,571\end{array}$ & $\begin{array}{r}12.1 \\
9.4\end{array}$ & $\begin{array}{l}1,732 \\
2,426\end{array}$ & $\begin{array}{l}3.0 \\
4.1\end{array}$ \\
\hline Unaffiliated & 31,544 & 9.7 & 29,934 & 9.4 & 29,183 & 9.3 & 3,714 & 6.4 \\
\hline Nonsectarian & 68,156 & 21.0 & 70,098 & 22.0 & 69,095 & 22.0 & 46,568 & 79.6 \\
\hline $\begin{array}{l}\text { Regular } \\
\text { Special emphasis } \\
\text { Special education }\end{array}$ & $\begin{array}{r}51,655 \\
7,289 \\
9,212\end{array}$ & $\begin{array}{r}15.9 \\
2.2 \\
2.8\end{array}$ & $\begin{array}{r}53,451 \\
7,691 \\
8,956\end{array}$ & $\begin{array}{r}16.8 \\
2.4 \\
2.8\end{array}$ & $\begin{array}{r}51,964 \\
7,485 \\
9,646\end{array}$ & $\begin{array}{r}16.5 \\
2.4 \\
3.1\end{array}$ & $\begin{array}{r}5,927 \\
12,165 \\
28,475\end{array}$ & $\begin{array}{l}10.1 \\
20.8 \\
48.7\end{array}$ \\
\hline $\begin{array}{l}{ }^{1} \text { The count for kindergarten } \\
\text { NOTE: Detail may not sum } \\
\text { private schools that provide }\end{array}$ & $\begin{array}{l}\text { students a } \\
\text { to totals be } \\
\text { instruction }\end{array}$ & $\begin{array}{l}\text { so include } \\
\text { ause of ro } \\
\text { or one or }\end{array}$ & $\begin{array}{l}\text { ansitional } \\
\text { ding or mi } \\
\text { re of grad }\end{array}$ & $\begin{array}{l}\text { I kindergart } \\
\text { issing value } \\
\text { les kinderg }\end{array}$ & $\begin{array}{l}\text { and trans } \\
\text { in cells wi } \\
\text { ten througl }\end{array}$ & $\begin{array}{l}\text { sitional first } \\
\text { ith too few }\end{array}$ & $\begin{array}{l}\text { ade stude } \\
\text { nple case } \\
\text { arable un }\end{array}$ & $\begin{array}{l}\text { ents. } \\
\text { es. These } \\
\text { ggraded I }\end{array}$ \\
\hline
\end{tabular}


Table 7. Average private school size, by school level and selected school characteristics:

United States, 2011-12

\begin{tabular}{|c|c|c|c|c|}
\hline \multirow{2}{*}{$\begin{array}{l}\text { School } \\
\text { characteristic }\end{array}$} & \multicolumn{4}{|c|}{ Average number of students } \\
\hline & Total & Elementary & Secondary & Combined \\
\hline Total & 145.6 & 107.8 & 283.0 & 190.1 \\
\hline \multicolumn{5}{|l|}{ Private school typology } \\
\hline Catholic & 280.6 & 227.2 & 535.5 & 336.2 \\
\hline Parochial & 250.4 & 237.8 & 439.5 & 348.4 \\
\hline Diocesan & 284.4 & 229.5 & 534.1 & 325.1 \\
\hline Private & 354.4 & 146.9 & 568.9 & 338.4 \\
\hline Other religious & 118.0 & 74.7 & 140.8 & 176.9 \\
\hline Conservative Christian & 137.8 & 91.2 & 129.4 & 164.5 \\
\hline Other affiliated & 159.1 & 106.7 & 164.5 & 272.3 \\
\hline Unaffiliated & 85.0 & 54.9 & 126.8 & 150.5 \\
\hline Nonsectarian & 91.0 & 47.3 & 103.7 & 195.4 \\
\hline Regular & 125.3 & 51.1 & 185.4 & 361.1 \\
\hline Special emphasis & 53.0 & 41.9 & 60.9 & 113.9 \\
\hline Special education & 64.8 & 47.7 & 42.9 & 73.9 \\
\hline \multicolumn{5}{|l|}{ Program emphasis } \\
\hline \multicolumn{5}{|l|}{ Regular elementary/ } \\
\hline secondary & 191.0 & 149.6 & 357.5 & 221.5 \\
\hline Montessori & 36.4 & 34.0 & $\ddagger$ & 77.4 \\
\hline Special program emphasis & 124.8 & 88.9 & 140.5 & 176.4 \\
\hline Special education & 63.1 & 46.7 & 42.8 & 71.7 \\
\hline Vocational/technical & $\ddagger$ & $\ddagger$ & $\ddagger$ & $\ddagger$ \\
\hline Alternative & 54.6 & 44.4 & 43.8 & 72.7 \\
\hline Early childhood & 15.4 & 15.3 & $\dagger$ & 23.7 \\
\hline \multicolumn{5}{|l|}{ Size (number of students) } \\
\hline Less than 50 & 19.9 & 18.6 & 21.4 & 24.0 \\
\hline $50-149$ & 91.4 & 93.3 & 88.9 & 88.6 \\
\hline 150-299 & 212.3 & 210.6 & 218.0 & 214.8 \\
\hline $300-499$ & 384.6 & 383.6 & 387.0 & 385.1 \\
\hline $500-749$ & 603.1 & 589.0 & 607.4 & 610.8 \\
\hline 750 or more & 1080.8 & 942.4 & 1049.6 & 1120.4 \\
\hline \multicolumn{5}{|l|}{ Region } \\
\hline Northeast & 144.8 & 101.4 & 285.1 & 196.8 \\
\hline Midwest & 138.1 & 113.6 & 350.8 & 143.0 \\
\hline South & 159.7 & 109.1 & 256.8 & 212.4 \\
\hline West & 135.5 & 106.1 & 236.2 & 180.7 \\
\hline \multicolumn{5}{|l|}{ Urbanicity type } \\
\hline City & 190.0 & 135.5 & 354.9 & 266.4 \\
\hline Suburban & 153.3 & 117.1 & 304.3 & 207.5 \\
\hline Town & 103.7 & 91.2 & 156.8 & 116.4 \\
\hline Rural & 88.1 & 51.0 & 142.9 & 132.6 \\
\hline
\end{tabular}

$\dagger$ Not applicable.

$\ddagger$ Reporting standards not met. There are fewer than 15 sample cases.

NOTE: These estimates include private schools that provide instruction for one or more of grades kindergarten through 12 (or comparable ungraded levels).

SOURCE: U.S. Department of Education, National Center for Education Statistics, Private School Universe Survey (PSS), 2011-2012. 
Table 8. Number and percentage distribution of private schools, by school size and selected school characteristics: United States, 2011-12

\begin{tabular}{|c|c|c|c|c|c|c|c|c|c|c|c|c|}
\hline \multirow{2}{*}{$\begin{array}{l}\text { School } \\
\text { characteristic }\end{array}$} & \multicolumn{2}{|c|}{$\begin{array}{l}\text { Less than } 50 \\
\text { students }\end{array}$} & \multicolumn{2}{|c|}{$\begin{array}{c}50-149 \\
\text { students }\end{array}$} & \multicolumn{2}{|c|}{$\begin{array}{l}150-299 \\
\text { students }\end{array}$} & \multicolumn{2}{|c|}{$\begin{array}{l}300-499 \\
\text { students }\end{array}$} & \multicolumn{2}{|c|}{$\begin{array}{l}500-749 \\
\text { students }\end{array}$} & \multicolumn{2}{|c|}{$\begin{array}{c}750 \text { or more } \\
\text { students }\end{array}$} \\
\hline & Number & Percent & Number & Percent & Number & Percent & Number & Percent & Number & Percent & Number & Percent \\
\hline Total & 13,459 & 43.6 & 7,667 & 24.8 & 5,488 & 17.8 & 2,447 & 7.9 & 1,103 & 3.6 & 698 & 2.3 \\
\hline \multicolumn{13}{|l|}{ Private school typology } \\
\hline Catholic & 399 & 5.8 & 1,559 & 22.7 & 2,688 & 39.1 & 1,323 & 19.3 & 606 & 8.8 & 297 & 4.3 \\
\hline Parochial & 104 & 3.6 & 686 & 23.6 & 1,310 & 45.0 & 589 & 20.2 & 178 & 6.1 & 44 & 1.5 \\
\hline Diocesan & 106 & 3.6 & 684 & 23.4 & 1,170 & 40.0 & 559 & 19.1 & 283 & 9.7 & 120 & 4.1 \\
\hline Private & 189 & 18.2 & 190 & 18.3 & 209 & 20.0 & 176 & 16.9 & 145 & 13.9 & 133 & 12.8 \\
\hline $\begin{array}{l}\text { Other religious } \\
\text { Conservative }\end{array}$ & 6,977 & 49.1 & 3,954 & 27.8 & 1,982 & 13.9 & 730 & 5.1 & 326 & 2.3 & 245 & 1.7 \\
\hline Christian & 1,751 & 38.3 & 1,511 & 33.0 & 796 & 17.4 & 290 & 6.3 & 135 & 2.9 & 92 & 2.0 \\
\hline Other affiliated & 1,074 & 35.1 & 973 & 31.8 & 593 & 19.4 & 227 & 7.4 & 106 & 3.5 & 88 & 2.9 \\
\hline Unaffiliated & 4,152 & 63.1 & 1,470 & 22.3 & 594 & 9.0 & 214 & 3.2 & 85 & 1.3 & 65 & 1.0 \\
\hline Nonsectarian & 6,083 & 62.2 & 2,154 & 22.0 & 817 & 8.4 & 393 & 4.0 & 172 & 1.8 & 157 & 1.6 \\
\hline Regular & 2,953 & 60.5 & 776 & 15.9 & 523 & 10.7 & 326 & 6.7 & 155 & 3.2 & 150 & 3.1 \\
\hline Special emphasis & 2,256 & 68.8 & 781 & 23.8 & 174 & 5.3 & 48 & 1.5 & $\ddagger$ & $\ddagger$ & $\ddagger$ & $\ddagger$ \\
\hline Special education & 873 & 54.1 & 597 & 37.0 & 121 & 7.5 & 19 & 1.2 & $\ddagger$ & $\ddagger$ & $\ddagger$ & $\ddagger$ \\
\hline \multicolumn{13}{|l|}{ School level } \\
\hline Elementary & 9,829 & 49.9 & 4,592 & 23.3 & 3,544 & 18.0 & 1,333 & 6.8 & 340 & 1.7 & 58 & 0.3 \\
\hline Secondary & 763 & 28.5 & 533 & 19.9 & 434 & 16.2 & 381 & 14.2 & 320 & 12.0 & 245 & 9.2 \\
\hline Combined & 2,866 & 33.8 & 2,542 & 29.9 & 1,510 & 17.8 & 732 & 8.6 & 443 & 5.2 & 395 & 4.7 \\
\hline \multicolumn{13}{|l|}{ Program emphasis } \\
\hline \multicolumn{13}{|l|}{ Regular elementary/ } \\
\hline secondary & 6,249 & 29.1 & 5,995 & 27.9 & 5,123 & 23.8 & 2,350 & 10.9 & 1,080 & 5.0 & 689 & 3.2 \\
\hline Montessori & 1,871 & 76.7 & 477 & 19.5 & 84 & 3.4 & $\ddagger$ & $\ddagger$ & 0 & 0.0 & 0 & 0.0 \\
\hline \multicolumn{13}{|l|}{ Special program } \\
\hline emphasis & 251 & 37.2 & 242 & 35.7 & 110 & 16.2 & 55 & 8.2 & $\ddagger$ & $\ddagger$ & $\ddagger$ & $\ddagger$ \\
\hline Special education & 1,032 & 55.5 & 671 & 36.1 & 133 & 7.1 & 21 & 1.1 & $\ddagger$ & $\ddagger$ & $\ddagger$ & $\ddagger$ \\
\hline Vocational/technical & $\ddagger$ & $\ddagger$ & $\ddagger$ & $\ddagger$ & $\ddagger$ & $\ddagger$ & $\ddagger$ & $\ddagger$ & $\ddagger$ & $\ddagger$ & $\ddagger$ & $\ddagger$ \\
\hline Alternative & 551 & 67.8 & 212 & 26.0 & 31 & 3.9 & $\ddagger$ & $\ddagger$ & $\ddagger$ & $\ddagger$ & $\ddagger$ & $\ddagger$ \\
\hline Early childhood & 3,503 & 97.8 & 69 & 1.9 & $\ddagger$ & $\ddagger$ & $\ddagger$ & $\ddagger$ & $\ddagger$ & $\ddagger$ & $\ddagger$ & $\ddagger$ \\
\hline \multicolumn{13}{|l|}{ Region } \\
\hline Northeast & 3,468 & 46.6 & 1,615 & 21.7 & 1,389 & 18.7 & 578 & 7.8 & 227 & 3.0 & 171 & 2.3 \\
\hline Midwest & 3,341 & 42.0 & 2,223 & 27.9 & 1,345 & 16.9 & 619 & 7.8 & 304 & 3.8 & 131 & 1.6 \\
\hline South & 3,761 & 40.9 & 2,378 & 25.8 & 1,602 & 17.4 & 795 & 8.6 & 387 & 4.2 & 280 & 3.0 \\
\hline West & 2,888 & 46.2 & 1,452 & 23.2 & 1,152 & 18.4 & 455 & 7.3 & 185 & 3.0 & 117 & 1.9 \\
\hline \multicolumn{13}{|l|}{ Urbanicity type } \\
\hline City & 3,399 & 34.0 & 2,419 & 24.2 & 2,260 & 22.6 & 1,041 & 10.4 & 511 & 5.1 & 375 & 3.7 \\
\hline Suburban & 4,736 & 43.4 & 2,414 & 22.1 & 2,037 & 18.7 & 1,015 & 9.3 & 458 & 4.2 & 251 & 2.3 \\
\hline Town & 1,147 & 39.5 & 1,110 & 38.3 & 464 & 16.0 & 138 & 4.7 & 34 & 1.2 & $\ddagger$ & $\ddagger$ \\
\hline Rural & 4,177 & 59.3 & 1,724 & 24.5 & 727 & 10.3 & 253 & 3.6 & 100 & 1.4 & 65 & 0.9 \\
\hline
\end{tabular}

$\ddagger$ Reporting standards not met. There are fewer than 15 sample cases.

NOTE: Detail may not sum to totals because of rounding or missing values in cells with too few sample cases. These estimates include private schools that provide instruction for one or more of grades kindergarten through 12 (or comparable ungraded levels).

SOURCE: U.S. Department of Education, National Center for Education Statistics, Private School Universe Survey (PSS), $2011-2012$. 
Table 9. Percentage distribution of private school students, by racial/ethnic background and selected school characteristics: United States, 2011-12

\begin{tabular}{|c|c|c|c|c|c|c|c|}
\hline \multirow[b]{2}{*}{$\begin{array}{l}\text { School } \\
\text { characteristic }\end{array}$} & \multirow[b]{2}{*}{ Hispanic } & \multicolumn{6}{|c|}{ Non-Hispanic } \\
\hline & & $\begin{array}{r}\text { American } \\
\text { Indian }\end{array}$ & Asian & Black & $\begin{array}{r}\text { Pacific } \\
\text { Islander }\end{array}$ & White & $\begin{array}{r}\text { Two or more } \\
\text { races }\end{array}$ \\
\hline Total & 10.0 & 0.5 & 5.5 & 8.9 & 0.6 & 71.2 & 3.4 \\
\hline \multicolumn{8}{|l|}{ Private school typology } \\
\hline Catholic & 14.0 & 0.5 & 4.7 & 7.4 & 0.6 & 69.3 & 3.5 \\
\hline Parochial & 14.9 & 0.3 & 4.7 & 6.6 & 0.6 & 69.8 & 3.2 \\
\hline Diocesan & 13.3 & 0.5 & 4.5 & 7.2 & 0.7 & 70.1 & 3.7 \\
\hline Private & 13.7 & 0.9 & 5.0 & 9.4 & 0.7 & 66.9 & 3.6 \\
\hline Other religious & 6.5 & 0.4 & 4.6 & 10.0 & 0.6 & 75.4 & 2.5 \\
\hline Conservative Christian & 7.9 & 0.5 & 4.8 & 11.3 & 0.5 & 72.3 & 2.7 \\
\hline Other affiliated & 6.4 & 0.4 & 5.1 & 8.6 & 0.7 & 75.9 & 2.9 \\
\hline Unaffiliated & 5.1 & 0.3 & 3.9 & 9.7 & 0.6 & 78.4 & 1.9 \\
\hline Nonsectarian & 7.7 & 0.6 & 8.9 & 10.3 & 0.5 & 67.1 & 4.8 \\
\hline Regular & 7.0 & 0.6 & 9.2 & 8.8 & 0.6 & 68.9 & 5.0 \\
\hline Special emphasis & 7.5 & 0.7 & 11.7 & 8.8 & 0.6 & 65.4 & 5.3 \\
\hline Special education & 12.7 & 0.8 & 3.0 & 21.0 & 0.3 & 59.5 & 2.8 \\
\hline \multicolumn{8}{|l|}{ School level } \\
\hline Elementary & 11.6 & 0.5 & 5.5 & 8.8 & 0.6 & 69.4 & 3.6 \\
\hline Secondary & 11.9 & 0.3 & 5.5 & 9.1 & 0.4 & 69.8 & 3.0 \\
\hline Combined & 7.0 & 0.5 & 5.4 & 9.1 & 0.7 & 74.1 & 3.2 \\
\hline \multicolumn{8}{|l|}{ Program emphasis } \\
\hline \multicolumn{8}{|l|}{ Regular elementary/ } \\
\hline secondary & 10.0 & 0.5 & 5.2 & 8.5 & 0.6 & 71.9 & 3.3 \\
\hline Montessori & 8.1 & 0.5 & 14.5 & 6.9 & 0.7 & 63.6 & 5.7 \\
\hline Special program emphasis & 5.9 & 0.5 & 11.8 & 6.7 & 0.4 & 69.7 & 4.9 \\
\hline Special education & 12.4 & 0.7 & 2.8 & 21.1 & 0.3 & 59.8 & 2.9 \\
\hline Vocational/technical & $\ddagger$ & $\ddagger$ & $\ddagger$ & $\ddagger$ & $\ddagger$ & $\ddagger$ & $\ddagger$ \\
\hline Alternative & 10.5 & 1.3 & 3.6 & 19.3 & 0.4 & 61.5 & 3.4 \\
\hline Early childhood & 10.1 & 0.5 & 7.2 & 11.4 & 1.0 & 66.2 & 3.5 \\
\hline \multicolumn{8}{|l|}{ Size (number of students) } \\
\hline Less than 50 & 9.4 & 0.8 & 4.9 & 12.9 & 0.5 & 68.6 & 2.9 \\
\hline $50-149$ & 9.8 & 0.8 & 5.1 & 13.2 & 0.5 & 67.7 & 2.9 \\
\hline 150-299 & 12.7 & 0.4 & 5.8 & 10.7 & 0.6 & 66.2 & 3.6 \\
\hline $300-499$ & 9.4 & 0.4 & 5.9 & 7.0 & 0.5 & 73.4 & 3.4 \\
\hline $500-749$ & 8.5 & 0.4 & 5.0 & 5.9 & 0.6 & 76.3 & 3.3 \\
\hline 750 or more & 8.1 & 0.3 & 5.5 & 5.9 & 0.9 & 75.6 & 3.6 \\
\hline \multicolumn{8}{|l|}{ Region } \\
\hline Northeast & 7.8 & 0.2 & 5.2 & 9.8 & 0.2 & 74.1 & 2.6 \\
\hline Midwest & 6.4 & 0.5 & 3.2 & 7.9 & 0.3 & 79.3 & 2.5 \\
\hline South & 10.3 & 0.3 & 4.0 & 11.3 & 0.3 & 71.1 & 2.6 \\
\hline West & 16.8 & 1.1 & 11.3 & 5.0 & 2.1 & 56.8 & 6.9 \\
\hline \multicolumn{8}{|l|}{ Urbanicity type } \\
\hline City & 12.6 & 0.4 & 6.3 & 11.4 & 0.7 & 64.5 & 4.0 \\
\hline Suburban & 9.7 & 0.3 & 5.9 & 8.6 & 0.6 & 71.7 & 3.3 \\
\hline Town & 6.1 & 0.8 & 2.2 & 3.3 & 0.7 & 84.7 & 2.1 \\
\hline Rural & 4.7 & 1.1 & 3.4 & 4.9 & 0.3 & 83.5 & 2.1 \\
\hline
\end{tabular}

$\ddagger$ Reporting standards not met. There are fewer than 15 sample cases.

NOTE: Detail may not sum to totals because of rounding or missing values in cells with too few sample cases. These estimates include private schools that provide instruction for one or more of grades kindergarten through 12 (or comparable ungraded levels). Hispanic includes Latino, American Indian includes Native American, Black includes African American, and Pacific Islander includes Native Hawaiian.

SOURCE: U.S. Department of Education, National Center for Education Statistics, Private School Universe Survey (PSS), $2011-2012$. 
Table 10. Percentage male enrollment in private schools and percentage distribution of private schools, by coeducational category and selected school characteristics: United States, by coeducational category and selected school characteristics: United States, 2011-12

\begin{tabular}{|c|c|c|c|c|}
\hline \multirow{2}{*}{$\begin{array}{l}\text { School } \\
\text { characteristic }\end{array}$} & \multirow{2}{*}{$\begin{array}{r}\text { Percent } \\
\text { male enrollment }\end{array}$} & \multicolumn{3}{|c|}{ Coeducational category } \\
\hline & & Coed schools & All-girls schools & All-boys schools \\
\hline Total & 51.3 & 95.8 & 1.8 & 2.3 \\
\hline \multicolumn{5}{|l|}{ Private school typology } \\
\hline Catholic & 50.8 & 93.7 & 3.5 & 2.9 \\
\hline Parochial & 50.4 & 98.8 & 0.9 & $\ddagger$ \\
\hline Diocesan & 50.5 & 97.7 & 1.2 & 1.1 \\
\hline Private & 52.3 & 68.0 & 17.0 & 15.0 \\
\hline Other religious & 51.0 & 96.4 & 1.4 & 2.2 \\
\hline Conservative Christian & 50.9 & 99.4 & $\ddagger$ & 0.4 \\
\hline Other affiliated & 50.6 & 92.4 & 3.4 & 4.2 \\
\hline Unaffiliated & 51.4 & 96.2 & 1.3 & 2.5 \\
\hline Nonsectarian & 53.0 & 96.6 & 1.3 & 2.2 \\
\hline Regular & 50.6 & 97.0 & 1.6 & 1.4 \\
\hline Special emphasis & 52.5 & 97.0 & 0.7 & 2.3 \\
\hline Special education & 68.2 & 94.2 & 1.5 & 4.3 \\
\hline \multicolumn{5}{|l|}{ School level } \\
\hline Elementary & 50.8 & 98.9 & 0.4 & 0.6 \\
\hline Secondary & 52.1 & 72.5 & 12.4 & 15.1 \\
\hline Combined & 51.5 & 96.1 & 1.7 & 2.2 \\
\hline \multicolumn{5}{|l|}{ Program emphasis } \\
\hline \multicolumn{5}{|l|}{ Regular elementary/ } \\
\hline secondary & 50.9 & 95.3 & 2.3 & 2.4 \\
\hline Montessori & 50.9 & 99.8 & $\ddagger$ & $\ddagger$ \\
\hline Special program emphasis & 50.2 & 95.7 & $\ddagger$ & 2.8 \\
\hline Special education & 67.6 & 93.6 & 1.6 & 4.8 \\
\hline Vocational/technical & $\ddagger$ & $\ddagger$ & $\ddagger$ & $\ddagger$ \\
\hline Alternative & 57.3 & 85.7 & 3.4 & 10.8 \\
\hline Early childhood & 44.6 & 99.8 & $\ddagger$ & 0.0 \\
\hline \multicolumn{5}{|l|}{ Size (number of students) } \\
\hline Less than 50 & 52.9 & 97.6 & 0.8 & 1.6 \\
\hline $50-149$ & 53.6 & 96.7 & 1.1 & 2.2 \\
\hline $150-299$ & 51.2 & 96.0 & 2.1 & 2.0 \\
\hline $300-499$ & 50.0 & 92.2 & 4.5 & 3.3 \\
\hline $500-749$ & 47.2 & 85.2 & 10.0 & 4.8 \\
\hline 750 or more & 53.9 & 80.9 & 5.4 & 13.7 \\
\hline \multicolumn{5}{|l|}{ Region } \\
\hline Northeast & 51.4 & 91.2 & 3.7 & 5.1 \\
\hline Midwest & 51.4 & 97.9 & 1.0 & 1.1 \\
\hline South & 51.4 & 97.0 & 1.3 & 1.7 \\
\hline West & 50.8 & 97.0 & 1.5 & 1.5 \\
\hline \multicolumn{5}{|l|}{ Urbanicity type } \\
\hline City & 51.0 & 93.6 & 3.2 & 3.2 \\
\hline Suburban & 51.3 & 96.2 & 1.7 & 2.1 \\
\hline Town & 51.8 & 99.1 & $\ddagger$ & 0.6 \\
\hline Rural & 52.1 & 97.1 & 0.7 & 2.2 \\
\hline
\end{tabular}

$\ddagger$ Reporting standards not met. There are fewer than 15 samples cases.

NOTE: Detail may not sum to totals because of rounding or missing values in cells with too few sample cases. These estimates include private schools that provide instruction for one or more of grades kindergarten through 12 (or comparable ungraded levels).

SOURCE: U.S. Department of Education, National Center for Education Statistics, Private School Universe Survey (PSS), 2011-2012. 
Table 11. Number and percentage distribution of private school teachers (headcount), by work status and selected characteristics: United States, 2011-12

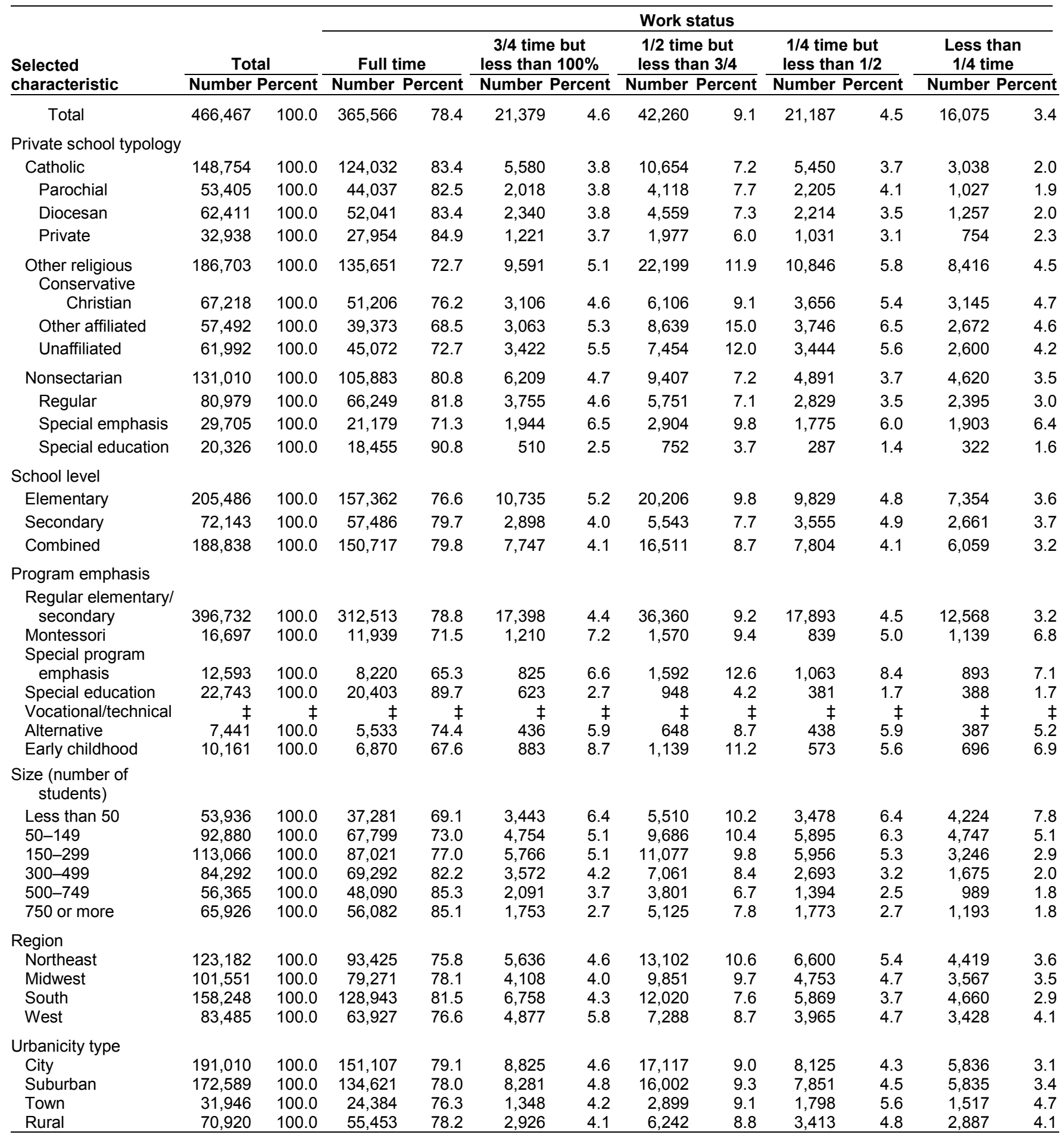

$\ddagger$ Reporting standards not met. There are fewer than 15 sample cases.

NOTE: Detail may not sum to totals because of rounding or missing values in cells with too few sample cases. The teacher estimates in this table are headcounts. These estimates include private schools that provide instruction for one or more of grades kindergarten through 12 (or comparable ungraded levels).

SOURCE: U.S. Department of Education, National Center for Education Statistics, Private School Universe Survey (PSS), $2011-2012$. 
Table 12. Pupil/teacher ratio of private schools, by school level and selected characteristics: United States, 2011-12

\begin{tabular}{|c|c|c|c|c|}
\hline \multirow{2}{*}{$\begin{array}{l}\text { Selected } \\
\text { characteristic }\end{array}$} & \multirow[b]{2}{*}{ Total } & \multicolumn{3}{|c|}{ School level } \\
\hline & & Elementary & Secondary & Combined \\
\hline Total & 10.7 & 11.5 & 11.6 & 9.4 \\
\hline \multicolumn{5}{|l|}{ Private school typology } \\
\hline Catholic & 14.0 & 14.6 & 13.6 & 11.0 \\
\hline Parochial & 14.8 & 15.0 & 13.4 & 12.7 \\
\hline Diocesan & 14.3 & 14.7 & 14.3 & 11.2 \\
\hline Private & 12.0 & 11.0 & 12.9 & 10.3 \\
\hline Other religious & 10.3 & 10.3 & 9.8 & 10.3 \\
\hline Conservative Christian & 10.6 & 10.2 & 11.8 & 10.7 \\
\hline Other affiliated & 9.9 & 10.0 & 9.3 & 9.9 \\
\hline Unaffiliated & 10.3 & 10.7 & 9.8 & 10.0 \\
\hline Nonsectarian & 7.4 & 7.1 & 6.9 & 7.8 \\
\hline Regular & 8.2 & 7.7 & 7.7 & 8.6 \\
\hline Special emphasis & 6.8 & 6.4 & 6.2 & 8.0 \\
\hline Special education & 5.4 & 5.2 & 5.1 & 5.4 \\
\hline \multicolumn{5}{|l|}{ Program emphasis } \\
\hline \multicolumn{5}{|l|}{ Regular elementary/ } \\
\hline secondary & 11.4 & 12.6 & 12.2 & 9.9 \\
\hline Montessori & 6.1 & 5.9 & $\ddagger$ & 8.4 \\
\hline Special program emphasis & 8.1 & 7.7 & 6.8 & 9.0 \\
\hline Special education & 5.4 & 5.3 & 5.2 & 5.4 \\
\hline Vocational/technical & $\ddagger$ & $\ddagger$ & $\ddagger$ & $\ddagger$ \\
\hline Alternative & 6.8 & 7.2 & 6.9 & 6.6 \\
\hline Early childhood & 6.4 & 6.4 & $\dagger$ & 4.6 \\
\hline \multicolumn{5}{|l|}{ Size (number of students) } \\
\hline Less than 50 & 5.9 & 6.2 & 4.8 & 5.4 \\
\hline $50-149$ & 8.7 & 9.5 & 7.5 & 7.7 \\
\hline 150-299 & 11.5 & 13.0 & 9.8 & 9.4 \\
\hline $300-499$ & 12.1 & 14.1 & 11.0 & 9.9 \\
\hline $500-749$ & 12.6 & 16.3 & 13.4 & 10.3 \\
\hline 750 or more & 12.2 & 16.1 & 14.3 & 11.0 \\
\hline \multicolumn{5}{|l|}{ Region } \\
\hline Northeast & 9.8 & 11.2 & 10.4 & 8.1 \\
\hline Midwest & 12.0 & 12.8 & 13.0 & 9.9 \\
\hline South & 10.1 & 10.4 & 11.6 & 9.7 \\
\hline West & 11.3 & 12.0 & 12.0 & 10.1 \\
\hline \multicolumn{5}{|l|}{ Urbanicity type } \\
\hline City & 11.0 & 11.7 & 12.6 & 9.4 \\
\hline Suburban & 10.7 & 11.7 & 11.6 & 9.1 \\
\hline Town & 10.6 & 11.4 & 10.7 & 9.8 \\
\hline Rural & 9.8 & 10.3 & 8.5 & 9.8 \\
\hline
\end{tabular}

† Not applicable.

¥ Reporting standards not met. There are fewer than 15 sample cases.

NOTE: These estimates include private schools that provide instruction for one or more of grades kindergarten through 12 (or comparable ungraded levels).

SOURCE: U.S. Department of Education, National Center for Education Statistics, Private School Universe Survey (PSS), 2011-2012. 
Table 13. Percentage of private schools with 12th-graders, number of graduates, graduation rate, and percentage of graduates who attended 4-year colleges, by selected characteristics: United States, 2010-11

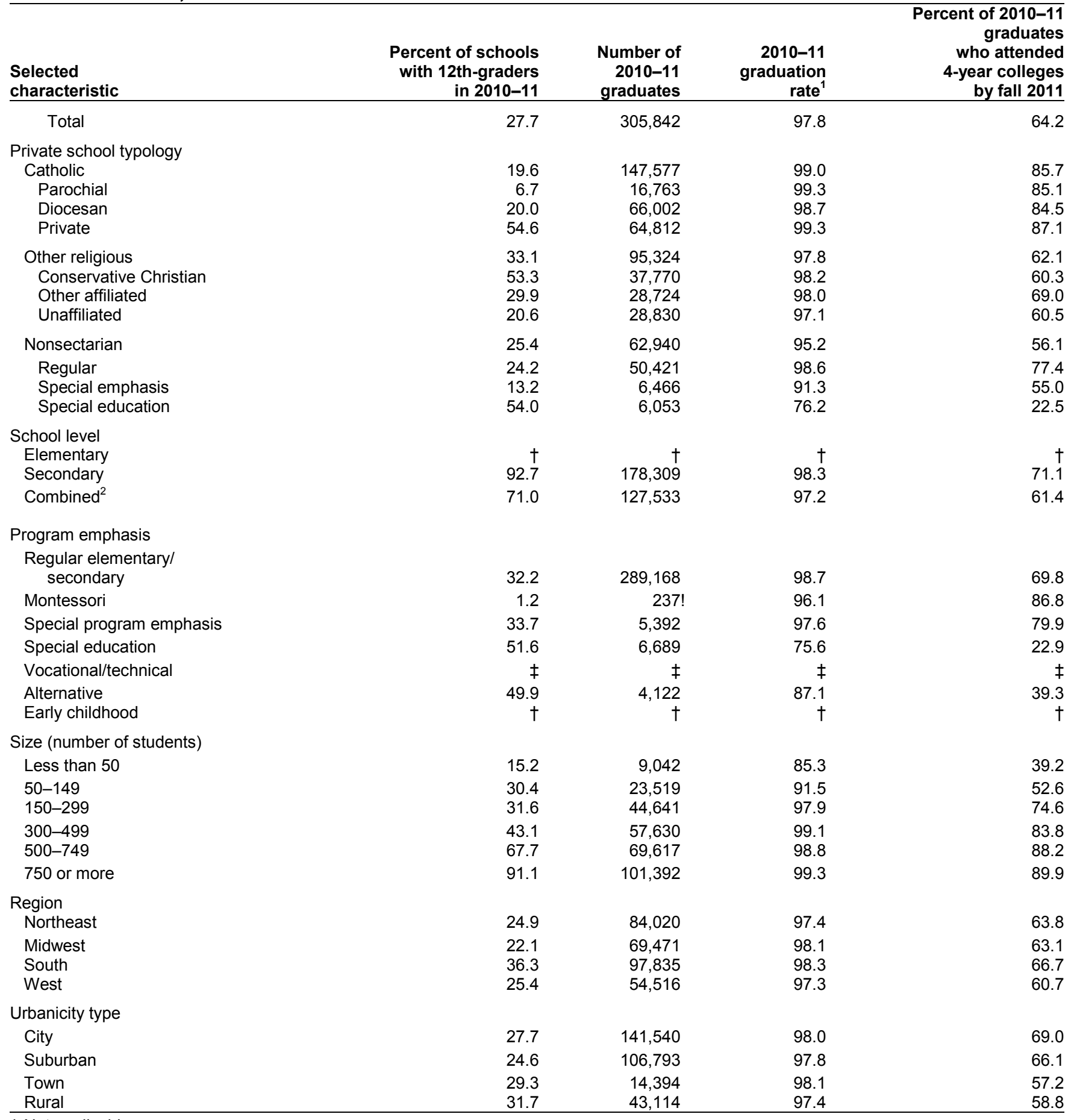

† Not applicable.

! Interpret data with caution. The coefficient of variation for this estimate is between 30 and 50 percent (i.e., the standard error is at least 30 percent and less than 50 percent of the estimate).

$\ddagger$ Reporting standards not met. There are fewer than 15 samples cases.

${ }^{1}$ The 2010-11 graduation rate is the percent of 12th-graders enrolled around October 1, 2010 who graduated in 2010-11.

${ }^{2}$ Includes the schools that reported 12th-grade enrollment for 2010-11, but were elementary in 2011-12 (less than 1 percent).

NOTE: Detail mail not sum to totals because of rounding or missing values in cells with too few sample cases. These estimates include private schools that provide instruction for one or more of grades kindergarten through 12 (or comparable ungraded levels).

SOURCE: U.S. Department of Education, National Center for Education Statistics, Private School Universe Survey (PSS), $2011-2012$. 
Table 14. Number of private schools, students, and teachers (headcount), by school membership in private school associations: United States, 2011-12

\begin{tabular}{|c|c|c|c|}
\hline Association & Schools & Students & Teachers \\
\hline Total & 30,861 & $4,494,845$ & 466,467 \\
\hline None & 10,212 & 710,000 & 82,644 \\
\hline \multicolumn{4}{|l|}{ Religious } \\
\hline Accelerated Christian Education & 1,073 & 43,026 & 6,060 \\
\hline American Association of Christian Schools & 719 & 77,367 & 8,929 \\
\hline Association of Christian Schools International & 2,948 & 524,299 & 53,757 \\
\hline Association of Christian Teachers and Schools & 195 & 26,440 & 2,704 \\
\hline Association of Classical and Christian Schools & 189 & 31,169 & 3,853 \\
\hline Christian Schools International & 321 & 69,740 & 6,698 \\
\hline Evangelical Lutheran Education Association & 163 & 15,217 & 1,477 \\
\hline Friends Council on Education & 67 & 16,859 & 2,402 \\
\hline General Conference of the Seventh-Day Adventist Church & 549 & 36,355 & 3,410 \\
\hline Islamic School League of America & 78 & 11,510 & 1,452 \\
\hline Jesuit Secondary Education Association & 50 & 44,268 & 3,411 \\
\hline National Association of Episcopal Schools & 282 & 80,019 & 9,970 \\
\hline National Catholic Educational Association & 5,740 & $1,682,048$ & 128,513 \\
\hline National Christian School Association & 141 & 33,637 & 3,181 \\
\hline National Society of Hebrew Day Schools & 262 & 76,865 & 10,193 \\
\hline Oral Roberts University Educational Fellowship & 53 & 10,403 & 1,053 \\
\hline The Jewish Community Day School Network & 108 & 20,588 & 3,662 \\
\hline Solomon Schechter Day School Assocation & 44 & 9,762 & 1,645 \\
\hline Southern Baptist Association of Christian Schools & 119 & 25,112 & 2,555 \\
\hline Other religious school associations & 1,945 & 340,521 & 33,626 \\
\hline \multicolumn{4}{|l|}{ Special emphasis } \\
\hline American Montessori Society & 1,334 & 61,729 & 11,567 \\
\hline Association Montessori International & 587 & 25,151 & 3,340 \\
\hline Other Montessori associations & 693 & 28,638 & 5,335 \\
\hline Association of Military Colleges and Schools & 21 & 4,335 & 583 \\
\hline Association of Waldorf Schools of North America & 112 & 16,491 & 2,536 \\
\hline National Association of Private Special Education Centers & 247 & 27,435 & 4,036 \\
\hline Other associations for exceptional children & 291 & 27,750 & 4,567 \\
\hline European Council for International Schools & $\ddagger$ & $\ddagger$ & $\ddagger$ \\
\hline National Association for the Education of Young Children & 1,378 & 106,953 & 13,158 \\
\hline National Association of Laboratory Schools & 21 & 2,613 & 378 \\
\hline National Coalition of Girls' Schools & 89 & 33,907 & 4,830 \\
\hline Other special emphasis school associations & 796 & 108,296 & 12,632 \\
\hline \multicolumn{4}{|l|}{ Other school associations or organizations } \\
\hline Alternative School Network & 25 & 2,317 & 449 \\
\hline National Association of Independent Schools & 1,472 & 601,970 & 79,874 \\
\hline State or regional independent school association & 2,259 & 656,934 & 77,862 \\
\hline National Coalition of Alternative Community Schools & 32 & 1,968 & 333 \\
\hline National Independent Private School Association & 201 & 34,374 & 4,269 \\
\hline The Association of Boarding Schools & 238 & 77,704 & 11,404 \\
\hline Other school associations & 3,587 & 707,505 & 74,208 \\
\hline
\end{tabular}

$\mp$ Reporting standards not met. There are fewer than 15 sample cases.

NOTE: Details do not sum to total because private schools may belong to more than one association. These estimates include private schools that provide instruction for one or more of grades kindergarten through 12 (or comparable ungraded levels).

SOURCE: U.S. Department of Education, National Center for Education Statistics, Private School Universe Survey (PSS), 2011-2012. 
Table 15. Number of private schools, students, full-time equivalent (FTE) teachers, and 2010-11 high school graduates, by state: United States, 2011-12

\begin{tabular}{|c|c|c|c|c|}
\hline State & Schools & Students & $\begin{array}{r}\text { FTE } \\
\text { teachers }\end{array}$ & $\begin{array}{r}\text { High school } \\
\text { graduates 2010-11 }\end{array}$ \\
\hline United States & 30,861 & $4,494,845$ & 420,880 & 305,842 \\
\hline $\begin{array}{l}\text { Alabama } \\
\text { Alaska } \\
\text { Arizona } \\
\text { Arkansas } \\
\text { California }\end{array}$ & $\begin{array}{r}402 \\
51 \\
341 \\
234 ! \\
3,479\end{array}$ & $\begin{array}{r}67,479 \\
4,087 \\
43,134 \\
25,164 \\
523,088\end{array}$ & $\begin{array}{r}6,208 \\
420 \\
3,809 \\
2,642 \\
44,268\end{array}$ & $\begin{array}{r}4,721 \\
218 \\
2,646 \\
1,495 \\
34,381\end{array}$ \\
\hline $\begin{array}{l}\text { Colorado } \\
\text { Connecticut } \\
\text { Delaware } \\
\text { District of Columbia } \\
\text { Florida }\end{array}$ & $\begin{array}{r}414 \\
406 \\
124 \\
79 \\
1,880\end{array}$ & $\begin{array}{r}45,697 \\
58,399 \\
21,667 \\
15,368 \\
284,238\end{array}$ & $\begin{array}{r}4,877 \\
7,077 \\
2,056 \\
1,957 \\
26,435\end{array}$ & $\begin{array}{r}2,892 \\
5,959 \\
1,774 \\
1,513 \\
20,060\end{array}$ \\
\hline $\begin{array}{l}\text { Georgia } \\
\text { Hawaii } \\
\text { Idaho } \\
\text { Illinois } \\
\text { Indiana }\end{array}$ & $\begin{array}{r}710 \\
134 \\
124 \\
1,572 \\
969\end{array}$ & $\begin{array}{r}120,488 \\
33,994 \\
11,262 \\
226,265 \\
111,872\end{array}$ & $\begin{array}{r}13,005 \\
3,013 \\
986 \\
19,149 \\
8,896\end{array}$ & $\begin{array}{r}7,761 \\
2,760 \\
583 \\
14,502 \\
5,622\end{array}$ \\
\hline $\begin{array}{l}\text { lowa } \\
\text { Kansas } \\
\text { Kentucky } \\
\text { Louisiana } \\
\text { Maine }\end{array}$ & $\begin{array}{l}300 \\
404 ! \\
329 \\
394 \\
157\end{array}$ & $\begin{array}{r}58,180 \\
39,016 \\
60,590 \\
112,645 \\
17,011\end{array}$ & $\begin{array}{l}4,702 \\
3,526 \\
5,239 \\
8,827 \\
2,001\end{array}$ & $\begin{array}{l}\ddagger \\
2,260 \\
4,127 \\
7,508 \\
2,601\end{array}$ \\
\hline $\begin{array}{l}\text { Maryland } \\
\text { Massachusetts } \\
\text { Michigan } \\
\text { Minnesota } \\
\text { Mississippi }\end{array}$ & $\begin{array}{l}736 \\
803 \\
791 \\
504 \\
221\end{array}$ & $\begin{array}{r}117,657 \\
112,684 \\
116,461 \\
76,693 \\
45,419\end{array}$ & $\begin{array}{r}12,429 \\
14,064 \\
9,290 \\
6,420 \\
4,122\end{array}$ & $\begin{array}{r}8,834 \\
10,134 \\
7,287 \\
4,705 \\
3,250\end{array}$ \\
\hline $\begin{array}{l}\text { Missouri } \\
\text { Montana } \\
\text { Nebraska } \\
\text { Nevada } \\
\text { New Hampshire }\end{array}$ & $\begin{array}{c}1,270 ! \\
111 \\
222 \\
157 \\
278\end{array}$ & $\begin{array}{r}115,553 \\
8,561 \\
36,163 \\
20,396 \\
21,264\end{array}$ & $\begin{array}{r}10,508 \\
976 \\
2,839 \\
1,594 \\
2,667\end{array}$ & $\begin{array}{r}7,527 \\
433 \\
2,304 \\
899 \\
2,522\end{array}$ \\
\hline $\begin{array}{l}\text { New Jersey } \\
\text { New Mexico } \\
\text { New York } \\
\text { North Carolina } \\
\text { North Dakota }\end{array}$ & $\begin{array}{r}1,289 \\
174 \\
1,931 \\
642 \\
46\end{array}$ & $\begin{array}{r}166,508 \\
19,360 \\
430,293 \\
106,661 \\
6,535\end{array}$ & $\begin{array}{r}16,847 \\
1,944 \\
41,454 \\
10,685 \\
588\end{array}$ & $\begin{array}{r}12,979 \\
1,275 \\
30,441 \\
6,312 \\
\mp\end{array}$ \\
\hline $\begin{array}{l}\text { Ohio } \\
\text { Oklahoma } \\
\text { Oregon } \\
\text { Pennsylvania } \\
\text { Rhode Island }\end{array}$ & $\begin{array}{r}968 \\
181 \\
432 \\
2,325 \\
144\end{array}$ & $\begin{array}{r}190,810 \\
30,063 \\
42,694 \\
240,993 \\
22,539\end{array}$ & $\begin{array}{r}15,269 \\
3,002 \\
3,818 \\
21,959 \\
2,362\end{array}$ & $\begin{array}{r}12,858 \\
1,764 \\
2,974 \\
16,366 \\
2,019\end{array}$ \\
\hline $\begin{array}{l}\text { South Carolina } \\
\text { South Dakota } \\
\text { Tennessee } \\
\text { Texas } \\
\text { Utah }\end{array}$ & $\begin{array}{r}378 \\
74 \\
512 \\
1,502 \\
160\end{array}$ & $\begin{array}{r}47,259 \\
10,266 \\
81,499 \\
223,469 \\
15,498\end{array}$ & $\begin{array}{r}5,001 \\
965 \\
8,150 \\
23,360 \\
1,620\end{array}$ & $\begin{array}{r}2,957 \\
653 \\
5,857 \\
12,838 \\
1,213\end{array}$ \\
\hline $\begin{array}{l}\text { Vermont } \\
\text { Virginia } \\
\text { Washington } \\
\text { West Virginia } \\
\text { Wisconsin } \\
\text { Wyoming }\end{array}$ & $\begin{array}{r}114 \\
755 \\
633 \\
124 \\
844 \\
38 \\
\end{array}$ & $\begin{array}{r}8,323 \\
98,439 \\
77,279 \\
11,890 \\
112,025 \\
1,947 \\
\end{array}$ & $\begin{array}{r}1,199 \\
10,981 \\
7,127 \\
1,126 \\
9,153 \\
265 \\
\end{array}$ & $\begin{array}{r}999 \\
6,402 \\
4,208 \\
660 \\
5,420 \\
\ddagger\end{array}$ \\
\hline
\end{tabular}

! Interpret data with caution. The coefficient of variation for this estimate is between 30 and 50 percent (i.e., the standard error is at least 30 percent and less than 50 percent of the estimate).

‡ Reporting standards not met. There are fewer than 15 samples cases or the coefficient of variation (CV) for this estimate is 50 percent or greater (i.e., the standard error is 50 percent or more of the estimate).

NOTE: Detail may not sum to totals because of rounding or missing values in cells with too few sample cases. These estimates include private schools that provide instruction for one or more of grades kindergarten through 12 (or comparable ungraded levels).

SOURCE: U.S. Department of Education, National Center for Education Statistics, Private School Universe Survey (PSS), $2011-2012$. 


\section{Appendix A-Glossary}

\section{Full-time equivalent (FTE)}

Full-time equivalent (FTE) quantifies teachers in proportion to a full-time teacher. Private school teachers are assigned an FTE based on respondent-provided information on the number of teachers in each full-time and part-time work status category in item 11.

- $\quad F T E=1.000:$ A teacher works full time.

- $F T E=.875:$ A teacher works at least 3/4 time but less than full time.

- $F T E=.625:$ A teacher works at least 1/2 time but less than 3/4 time.

- $F T E=.375:$ A teacher works at least 1/4 time but less than $1 / 2$ time.

- $F T E=.125:$ A teacher works less than $1 / 4$ time.

The number of FTE teachers is calculated by summing the number of FTEs in each work status category.

\section{Graduation rate}

The graduation rate is the number of 2010-11 graduates divided by the number of 12th-grade students enrolled around October 1, 2010.

\section{Kindergarten-terminal school}

A school is kindergarten-terminal (k-terminal) if kindergarten is the highest grade offered at the school.

\section{Private school}

A private school is a school that is not supported primarily by public funds. It must provide classroom instruction for one or more of grades K-12 (or comparable ungraded levels), and have one or more teachers. Organizations or institutions that provide support for home schooling but do not offer classroom instruction for students are not included.

\section{Program emphasis}

Private schools are classified by respondents in item 12a according to one of seven types of program emphasis that best describes the school:

- Regular: The PSS questionnaire does not provide a definition of this term. Regular schools do not specialize in special, vocational/technical, or alternative education; or in having a Montessori or special program emphasis, although they may offer these programs in addition to the regular curriculum.

- Montessori: The PSS questionnaire does not provide a definition of this term. Montessori schools provide instruction using Montessori teaching methods. 
- Special program emphasis: A science/mathematics school, a performing arts high school, a foreign language immersion school, and a talented/gifted school are examples of schools that offer a special program emphasis.

- Special education: Special education schools primarily serve students with disabilities.

- Career/Technical/Vocational: Career/Technical/Vocational schools primarily serve students who are being trained for occupations.

- Alternative: Alternative schools provide nontraditional education. They fall outside the categories of regular, special education, and vocational education.

- Early childhood: Early childhood program schools serve students in prekindergarten, kindergarten, transitional (or readiness) kindergarten, and/or transitional first (or prefirst) grade.

\section{Region}

Private schools are assigned to one of four geographic regions:

- Northeast: Maine, New Hampshire, Vermont, Massachusetts, Rhode Island, Connecticut, New York, New Jersey, and Pennsylvania;

- Midwest: Ohio, Indiana, Illinois, Michigan, Wisconsin, Minnesota, Iowa, Missouri, North Dakota, South Dakota, Nebraska, and Kansas;

- South: Delaware, Maryland, District of Columbia, Virginia, West Virginia, North Carolina, South Carolina, Georgia, Florida, Kentucky, Tennessee, Alabama, Mississippi, Arkansas, Louisiana, Oklahoma, and Texas; and

- West: Montana, Idaho, Wyoming, Colorado, New Mexico, Arizona, Utah, Nevada, Washington, Oregon, California, Alaska, and Hawaii.

\section{School level}

Private schools are classified using respondent-provided highest and lowest grades in the school in item 5 according to one of three instructional levels:

- Elementary: A school is elementary if it has one or more of grades $\mathrm{K}-6$ and does not have any grade higher than grade 8 ; for example, schools with grades $\mathrm{PK}-\mathrm{K}, \mathrm{K}-6,1-3$, or 6-8 are classified as elementary schools.

- Secondary: A school is secondary if it has one or more of grades 7-12 and does not have any grade lower than grade 7; for example, schools with grades 9-12, 7-8, 10-12, or 7-9 are classified as secondary schools.

- Combined: A school is classified as combined if it has one or more of grades $\mathrm{K}-6$ and one or more of grades 9-12; for example, schools with grades $\mathrm{K}-12,6-12,6-9$, or $1-12$ are classified as having combined grades. Schools in which all students are ungraded (i.e., not classified by standard grade levels) are also classified as combined. 


\section{Teacher}

Any full-time or part-time school staff member who teaches one or more regularly scheduled classes in any of grades $\mathrm{K}-12$ (or comparable ungraded levels).

\section{Transitional Kindergarten or Transitional First}

Extra year of school for either kindergarten-age children who are judged not ready for kindergarten, or for children who have attended kindergarten but have been judged not ready for the first grade.

\section{Typology}

Private schools are assigned to one of three major categories (Catholic, other religious, or nonsectarian) and, within each major category, one of three subcategories based on the school's religious affiliation provided by respondents in item 14 .

- Catholic: Catholic schools are categorized according to governance, provided by Catholic school respondents in item 14c, into parochial, diocesan, and private schools.

- Other religious: Other religious schools have a religious orientation or purpose, but are not Roman Catholic. Other religious schools are categorized according to religious association membership, provided by respondents in item 15, into Conservative Christian, other affiliated and unaffiliated schools. Conservative Christian schools are those "Other religious" schools with membership in at least one of four associations: Accelerated Christian Education, American Association of Christian Schools, Association of Christian Schools International, or Oral Roberts University Education Fellowship. Other affiliated schools are those "Other religious" schools not classified as Conservative Christian with membership in at least 1 of 11 associations-Association of Christian Teachers and Schools, Christian Schools International, Evangelical Lutheran Education Association, Friends Council on Education, General Conference of the Seventh-Day Adventist Church, Islamic School League of America, National Association of Episcopal Schools, National Christian School Association, National Society for Hebrew Day Schools, Solomon Schechter Day Schools, Southern Baptist Association of Christian Schools - or indicating membership in "other religious school associations." Unaffiliated schools are those "Other religious" schools that have a religious orientation or purpose, but are not classified as Conservative Christian or affiliated.

- Nonsectarian: Nonsectarian schools do not have a religious orientation or purpose and are categorized according to program emphasis, provided by respondents in item 12a, into regular, special emphasis, and special education schools. Regular schools are those that have a regular elementary/secondary or early childhood program emphasis. Special emphasis schools are those that have a Montessori, career/technical/vocational, alternative, or special program emphasis. Special education schools are those that have a special education program emphasis.

\section{Ungraded students}

Ungraded students are those who are not assigned to a particular grade level (kindergarten, first grade, second grade, etc.). For example, special education centers and alternative schools often 
classify their students as ungraded. Students in Montessori schools are also considered ungraded if the school assigns them to "primary" and "intermediate" levels instead of specific grades.

\section{Urbanicity type}

Urbanicity type is derived from the 12-category, urban-centric locale code (ULOCALE). The urban-centric locale code is based on the school's physical address (or mailing address if the physical address is not reported) and is a measure of a school's location relative to populous areas. For this report, the urban-centric locale codes were aggregated into four urbanicity types:

- City: The territory inside an urbanized area and inside a principal city. (ULOCALE $=11$, 12 , or 13$)$;

- Suburb: The territory outside a principal city and inside an urbanized area. (ULOCALE = 21,22 , or 23 );

- Town: Territory inside an urban cluster. (ULOCALE =31, 32, or 33); and

- Rural: Census defined rural territory. (ULOCALE $=41,42$, or 43). 


\section{Appendix B-Technical Notes}

\section{Background}

The Private School Universe Survey (PSS) is conducted by the National Center for Education Statistics (NCES) of the U.S. Department of Education in order to collect basic information on private elementary and secondary schools in the United States. The PSS grew out of a proposal in 1988 to develop a private school data collection that would improve on the sporadic collection of private school data dating back to 1890 and improve on commercially available private school sampling frames. The PSS is currently designed to generate biennial data on the total number of private schools, teachers, and students, and to build a universe of private schools to serve as a sampling frame for the NCES sample surveys. The PSS data were first collected by the U.S. Census Bureau in the 1989-90 school year, with data collections every 2 years since.

\section{Target Population}

The target population for the PSS is all schools located in the 50 states and the District of Columbia that are not supported primarily by public funds, provide classroom instruction for one or more of grades kindergarten through 12 (or comparable ungraded levels), and have one or more teachers. Organizations or institutions that provide support for home schooling, but do not provide classroom instruction, are not included.

\section{Content}

The 2011-12 PSS instrument consisted of a single school questionnaire designed to be filled out by school administrators and is available on the PSS website at http://nces.ed.gov/surveys/pss. Data collected included enrollment by grade, enrollment by race/ethnicity and sex, number of high school graduates from the previous year, number of teachers, program emphasis, school religious orientation or affiliation, association membership, existence and type of kindergarten program, number of days in the school year and length of the school day, and whether the school has a library media center.

\section{Frame Creation}

Because the PSS attempts to include every private school in the United States, a universe list of private schools meeting the PSS definition had to be created for the 2011-12 PSS. Since 1983, NCES has used a dual-frame approach for building its private school universe (Beller 1984). The dual frame consists of a list frame and an area frame.

\section{List Frame}

The list-building component was the primary means for improving coverage of private schools. The basis for the 2011-12 PSS list frame was the 29,448 private schools from both the 2009-10 PSS list frame and the certainty portion of the 2009-10 area frame. This became the base list against which all other submissions were compared. In order to provide coverage of private schools founded since 2009 and to improve coverage of private schools existing in 2009, NCES solicited membership lists from 31 private school associations and religious denominations. NCES received and processed 28 of these lists. 
The departments of education from 50 states and the District of Columbia provided lists of private schools. All 51 department of education lists were processed. Additional private schools that contacted NCES to be included in the PSS were added as list frame cases.

Beginning in 1995, the PSS private school definition was expanded to include schools for which kindergarten is the highest grade. In 2011, a separate list-building operation (Early Childhood Operation) was conducted to identify schools for which kindergarten was the highest grade (kindergarten terminal or k-terminal schools). Requests for lists of programs that might include a kindergarten were made to sources, other than state departments of education, in all 50 states and the District of Columbia, including state departments of health or recreation; state child care licensing agencies; and child care referral agencies. In 2011, a total of 30 of these lists were received and 19 lists were processed. Due to resource constraints not all received early childhood lists were processed in 2011.

Schools on private school association membership lists, the state lists, and the early childhood lists were compared to the base list, and any school that did not match a school on the base list was added to the universe list. Additionally, programs identified in the 2009-10 PSS as prekindergarten only were included in the 2011-12 list frame. This was done in case any of these programs included at least a kindergarten in the 2011-12 school year. A total of 37,524 schools were included in the 2011-12 list frame (table B-1).

Table B-1. Number of responding and nonresponding schools, out-of-scope cases, and school response rate, by frame: 2011-12

\begin{tabular}{lccc}
\hline Response status & List frame & Area frame & Total frame \\
\hline$\quad$ Total & & & \\
Base-weighted & 37,524 & 6,418 & 43,942 \\
Unweighted & 37,524 & 1,801 & 39,325 \\
Response & & & \\
$\quad$ Base-weighted & 26,730 & 1,615 & 28,345 \\
$\quad$ Unweighted & 26,730 & 253 & 26,983 \\
Nonresponse & & & \\
$\quad$ Base-weighted & 2,201 & 326 & 2,527 \\
$\quad$ Unweighted & 2,201 & 111 & 2,312 \\
Out-of-scope & & & \\
$\quad$ Base-weighted & 8,593 & 4,478 & 13,071 \\
$\quad$ Unweighted & 8,593 & 1,437 & 10,030 \\
Response rate & & & \\
$\quad$ Base-weighted & 92.4 & 83.2 & 91.8 \\
$\quad$ Unweighted & 92.4 & 69.5 & 92.1 \\
\hline NOTE: Weighted & & &
\end{tabular}

NOTE: Weighted using the inverse of the probability of selection.

SOURCE: U.S. Department of Education, National Center for Education Statistics, Private School Universe Survey (PSS), 2011-12.

\section{Area Frame}

To identify private schools that may have been overlooked in the list-building component, a group of geographic areas was selected to be searched for private schools. The United States is divided by the Census Bureau into 2,062 primary sampling units (PSUs), each PSU consisting of 
block listing of all private schools in a sample of PSUs was not attempted. Rather, regional office field staff created the frame by using such sources as yellow pages, local Catholic dioceses, local education agencies, and local government offices. The Census Bureau also obtained, for the first time, a list of eligible private schools from the InfoUSA database. ${ }^{1}$ Once the area search lists were constructed, they were matched with the NCES private school universe list. Schools that did match the universe list were deleted from the area frame. A total of 1,801 schools were added to the universe from the area frame (table B-1).

\section{Data Collection}

The 2011-12 Private School Universe Survey (PSS) data collection began on September 30, 2011 with the mailout of a letter to most private schools describing how to respond to the 201112 PSS online, and including a userid and password for the online option (Amish and Mennonite schools were excluded from this mailout). The first mailout of the PSS questionnaires (to all private schools) occurred on October 14, 2011, and a second mailout of questionnaires (to nonresponding schools only) occurred on November 30, 2011. Each of these mailings also included the instructions, userid, and password for the online option. A reminder postcard was sent 1 week after each questionnaire mailout. Follow-up of nonresponding schools was conducted first by computer-assisted telephone interviewing, and then by Census Bureau field representatives. Data collection for the 2011-12 PSS ended on May 22, 2012.

Of the 39,325 schools included in the 2011-12 PSS, 10,030 cases were considered as out-ofscope (not eligible for the PSS). A total of 26,983 private schools completed a PSS interview (15.8 percent completed online), while 2,312 schools refused to participate resulting in an unweighted response rate of 92.1 percent. $^{2}$

\section{Quality Control and Editing}

Data from the interviews went through several edits; the edits consisted of

- range checks to eliminate out-of-range entries;

- consistency checks to compare data in different fields for consistency;

- edits to verify that skip patterns on the questionnaire had been followed;

- logic edits where data were added to the questionnaire using information from the same PSS record or from the previous PSS record.

\section{Logic Edits}

In the logic edits, data were added to questionnaire records when data were missing or incomplete. The sources for these added data were as follows:

- Other items on the 2011-12 PSS-Based on entries from related items on the questionnaire, assumptions were made about how the respondent should have answered items with missing values. For example, if the respondent did not answer item 10a (length of school day for kindergarten, transitional kindergarten, transitional first grade)

\footnotetext{
${ }^{1}$ See http://www.infousa.com/.

2 The response rate is calculated as follows: number of responding schools divided by the number of responding schools plus the number of nonresponding schools.
} 
and item 5 indicated that the school did not have students enrolled in any of those grades, zero (school does not offer kindergarten) was assigned to item 10a.

- Data from the 2009-10 PSS-Data from the 2009-10 PSS were used to fill the items with missing values whenever possible. For example, if the type of school was not reported in item 12 and it had been reported on the 2009-10 PSS, the 2009-10 response was copied to item 12.

In addition to filling items where values were missing, some inconsistencies between items were corrected by ratio adjustment. For records where the number of students in item 7 (students by race) did not equal the enrollment in item 6, excluding prekindergarten, the item 7 entries were adjusted to be consistent with item 6 (i.e., each entry in item 7 was multiplied by the ratio of the student count in item 7 to the enrollment in item 6 , excluding prekindergarten).

\section{Imputation}

\section{Item Response}

The weighted item response rates for the variables used in this report are all greater than 95 percent. Values were imputed to items with missing data to compensate for item nonresponse.

\section{Imputation}

Items still blank after the logic edit were imputed using donor imputation. The imputation process used a hot-deck imputation methodology by extracting data from the record for a school (donor) similar to the nonrespondent. Variables that describe certain characteristics of the schools (e.g., religious affiliation, size, instructional level) were created and used to sort the records and to match incomplete records to those with complete data. Items $13 \mathrm{~b}$ (whether or not the school was located in a private home that was used primarily as a family residence) and 15 (school association membership) did not go through the donor imputation program. If, after the first stage, the item was still incomplete, the assumption was made for item $13 \mathrm{~b}$ that the school was not in a private home, and for item 15 that the school did not belong to any associations. Items $14 \mathrm{a}$ and $14 \mathrm{c}$ (religious orientation and affiliation) did not go through the donor imputation program. Rather, if values were still missing after the first-stage imputation, the records were reviewed and imputed by an analyst.

\section{Analyst Imputation}

For a few items, there were cases where entries were imputed by analysts during data review. The data record, sample file record, and the questionnaire were reviewed and an entry consistent with the information from those sources was imputed. This procedure was used when (1) there was no suitable donor found, (2) the computer method produced an imputed entry that was unacceptable, and (3) the nature of the item required an actual review of the data rather than a computer-generated value.

\section{Weighting}

The survey data from the area-frame component were weighted to reflect the sampling rates (probability of selection) of the PSUs. There were 26,983 interviews and 2,312 cases that were noninterviews. After applying the PSU weight, these became 28,345 interviews and 2,527 noninterviews - the weighted response rate was 91.8 percent (table B-1). 


\section{Sampling Error}

The standard error indicates how much variability there is in the population of possible estimates of a parameter for a given sample size. Standard errors are used as a measure of the precision expected from a particular sample. If all possible samples were surveyed under similar conditions, intervals of 1.96 standard errors below to 1.96 standard errors above a particular statistic would include the true population parameter being estimated in about 95 percent of the samples. This is a 95 percent confidence interval.

Estimates of standard errors were computed using a technique known as half-sample replication. As with any replication method, half-sample replication involves constructing a number of subsamples (replicates) from the full sample and computing the statistic of interest for each replicate. The mean squared error of the replicate estimates around the full sample provides an estimate of the variance of the statistic (Wolter 1985). A computer program (SUDAAN), developed by Research Triangle Institute, was used to calculate the estimates of standard errors. For PSS, only the area frame contributes to the standard error. Since all list-frame cases are included in the PSS, the list-frame component of the standard error is always zero (0). Standard errors for statistics in each table are presented in corresponding tables in appendix C.

\section{Tests of Significance}

The tests of significance used in this analysis are based on Student's $t$ statistics. The $t$ statistic between estimates from various independent subgroups presented in the tables can be computed by using the following formula:

$$
t=\frac{x_{1}-x_{2}}{\sqrt{S E_{1}^{2}+S E_{2}^{2}}}
$$

where $x_{1}$ and $x_{2}$ are the estimates to be compared (e.g., the means of two groups) and $S E_{1}$ and $S E_{2}$ are their corresponding standard errors.

As the number of comparisons that are conducted at the same significance increases, it becomes more likely that at least one of the estimated differences becomes significant merely by chance, that is, will be erroneously identified as significantly different from zero. Even when there is no statistical difference between the estimates or percentages being compared, there is a 5 percent chance of getting a significant $t$ value of 1.96 from sampling error alone. As the number of comparisons increases, the incidence of this type of error also increases.

\section{Nonsampling Error}

Survey estimates are also subject to errors of reporting and errors made in the collection of the data. These errors, called nonsampling errors, can sometimes bias the data. Nonsampling errors may include such things as differences in the respondents' interpretation of the meaning of the questions, differences related to the particular time the survey was conducted, or errors in data preparation. While sampling theory can be used to estimate the sampling variability of an estimate, nonsampling errors usually require either an experiment conducted as part of the data collection procedure or use of data external to the study. 
Undercoverage in the list and area frames is another possible source of nonsampling error. To reduce the possible bias caused by undercoverage in the list frame, the area frame was used to complement the list frame through the identification of schools missing from the list frame.

\section{Publication Criteria for PSS Estimates}

NCES criteria for the publication of an estimate are based on two category types: (1) surveys based on a sample from the frame or (2) surveys based on the entire frame (universe surveys). To publish an estimate for a sample survey, at least 30 cases must be used in developing the estimate; for a universe survey, at least three cases must be used in developing the estimate. PSS has pieces of both categories: (1) an area-frame sample of 124 PSUs, which collects data on schools not on the list frame, and (2) a complete census of schools belonging to the list frame. Since a significant component of a PSS estimate comes from the census of the list frame, the criterion of at least 30 cases seems unnecessarily high. However, since the area-frame component of an estimate can have a large sampling error, some indication of a large sampling error is appropriate. Based on these two concerns, the estimates reported in a PSS table must be based on at least 15 schools. Some of the estimates in this report are footnoted with "Interpret data with caution. The coefficient of variation is between 25 and 30 percent." The coefficient of variation measures the precision of an estimate and is defined as the standard error of an estimate divided by the value of the estimate. 
Appendix C-Standard Error Tables 
Table C-1. Standard errors for number and percentage distribution of private schools, students, and full-time equivalent (FTE) teachers, by school characteristics: United States, 2011-12

\begin{tabular}{|c|c|c|c|c|c|c|}
\hline \multirow{2}{*}{$\begin{array}{l}\text { School } \\
\text { characteristic }\end{array}$} & \multicolumn{2}{|c|}{ Schools } & \multicolumn{2}{|c|}{ Students } & \multicolumn{2}{|c|}{ FTE teachers } \\
\hline & Number & Percent & Number & Percent & Number & Percent \\
\hline Total & 542.4 & $\dagger$ & $25,138.6$ & $\dagger$ & $1,836.0$ & $\dagger$ \\
\hline \multicolumn{7}{|l|}{ Private school typology } \\
\hline Catholic & 31.1 & 0.4 & $14,630.3$ & 0.3 & $1,069.0$ & 0.2 \\
\hline Parochial & 15.7 & 0.2 & $3,325.6$ & 0.1 & 202.5 & 0.1 \\
\hline Diocesan & 27.8 & 0.2 & $14,320.0$ & 0.3 & $1,052.2$ & 0.2 \\
\hline Private & 17.5 & 0.1 & 217.6 & 0.0 & 65.4 & 0.0 \\
\hline Other religious & 550.9 & 1.0 & $21,908.0$ & 0.3 & $1,568.3$ & 0.3 \\
\hline Conservative Christian & 127.3 & 0.3 & $4,641.5$ & 0.1 & 575.1 & 0.1 \\
\hline Other affiliated & 17.1 & 0.2 & $2,397.8$ & 0.1 & 402.9 & 0.1 \\
\hline Unaffiliated & 458.3 & 1.1 & $20,414.6$ & 0.4 & $1,247.3$ & 0.3 \\
\hline Nonsectarian & 87.1 & 0.6 & $4,512.6$ & 0.2 & 567.0 & 0.2 \\
\hline Regular & 71.1 & 0.4 & $4,303.4$ & 0.1 & 496.7 & 0.1 \\
\hline Special emphasis & 23.2 & 0.2 & 870.4 & 0.0 & 140.0 & 0.0 \\
\hline Special education & 21.9 & 0.1 & 693.6 & 0.0 & 87.1 & 0.0 \\
\hline \multicolumn{7}{|l|}{ School level } \\
\hline Elementary & 414.1 & 0.4 & $8,772.0$ & 0.2 & 854.8 & 0.2 \\
\hline Secondary & 30.1 & 0.2 & $14,401.0$ & 0.3 & $1,055.3$ & 0.2 \\
\hline Combined & 188.3 & 0.4 & $17,100.8$ & 0.3 & 917.9 & 0.1 \\
\hline \multicolumn{7}{|l|}{ Program emphasis } \\
\hline \multicolumn{7}{|l|}{ Regular elementary/ } \\
\hline secondary & 539.9 & 0.6 & $25,216.0$ & 0.1 & $1,800.6$ & 0.1 \\
\hline Montessori & 19.6 & 0.1 & 781.7 & 0.0 & 130.3 & 0.0 \\
\hline Special program emphasis & 7.5 & 0.0 & 240.2 & 0.0 & 29.1 & 0.0 \\
\hline Special education & 28.0 & 0.1 & 943.4 & 0.0 & 158.3 & 0.0 \\
\hline Vocational/technical & $\dagger$ & $\dagger$ & $\dagger$ & $\dagger$ & $\dagger$ & $\dagger$ \\
\hline Alternative & 1.9 & 0.0 & 112.8 & 0.0 & 13.8 & 0.0 \\
\hline Early childhood & 43.8 & 0.2 & 627.9 & 0.0 & 99.1 & 0.0 \\
\hline \multicolumn{7}{|l|}{ Size (number of students) } \\
\hline Less than 50 & 521.2 & 1.0 & $7,510.8$ & 0.2 & $1,107.5$ & 0.2 \\
\hline $50-149$ & 114.1 & 0.5 & $7,353.2$ & 0.2 & 782.7 & 0.2 \\
\hline 150-299 & 14.7 & 0.3 & $2,944.8$ & 0.2 & 323.2 & 0.1 \\
\hline $300-499$ & 7.0 & 0.1 & $2,848.3$ & 0.1 & 269.2 & 0.1 \\
\hline $500-749$ & 27.0 & 0.1 & $14,312.1$ & 0.3 & $1,051.2$ & 0.2 \\
\hline 750 or more & 9.9 & 0.1 & $17,907.6$ & 0.3 & 696.4 & 0.2 \\
\hline \multicolumn{7}{|l|}{ Region } \\
\hline Northeast & 67.8 & 0.5 & $17,327.1$ & 0.3 & 679.5 & 0.2 \\
\hline Midwest & 532.7 & 1.3 & $18,087.1$ & 0.3 & $1,667.9$ & 0.3 \\
\hline South & 76.4 & 0.5 & $2,335.7$ & 0.2 & 363.2 & 0.2 \\
\hline West & 6.7 & 0.4 & 302.9 & 0.1 & 30.5 & 0.1 \\
\hline \multicolumn{7}{|l|}{ Urbanicity type } \\
\hline City & 51.7 & 0.6 & $14,550.9$ & 0.3 & $1,121.0$ & 0.2 \\
\hline Suburban & 69.7 & 0.6 & $5,325.6$ & 0.2 & 591.4 & 0.2 \\
\hline Town & 103.7 & 0.2 & $3,128.6$ & 0.1 & 598.3 & 0.1 \\
\hline Rural & 452.8 & 1.1 & $19,863.7$ & 0.4 & $1,123.5$ & 0.2 \\
\hline
\end{tabular}

† Not applicable.

SOURCE: U.S. Department of Education, National Center for Education Statistics, Private School Universe Survey (PSS), 2011-2012. 
Table C-2. Standard errors for number and percentage distribution of private schools, students, and full-time equivalent (FTE) teachers, by religious or nonsectarian orientation of school:

United States, 2011-12

\begin{tabular}{|c|c|c|c|c|c|c|}
\hline \multirow{2}{*}{$\begin{array}{l}\text { Religious or } \\
\text { nonsectarian orientation }\end{array}$} & \multicolumn{2}{|c|}{ Schools } & \multicolumn{2}{|c|}{ Students } & \multicolumn{2}{|c|}{ FTE teachers } \\
\hline & Number & Percent & Number & Percent & Number & Percent \\
\hline Total & 542.4 & $\dagger$ & $25,138.6$ & $\dagger$ & $1,836.0$ & \\
\hline Religious orientation & 551.6 & 0.63 & $25,845.9$ & 0.15 & $1,874.4$ & 0.17 \\
\hline Roman Catholic & 31.1 & 0.41 & $14,630.3$ & 0.28 & $1,069.0$ & 0.22 \\
\hline African Methodist Episcopal & $\dagger$ & $\dagger$ & $\dagger$ & $\dagger$ & $\dagger$ & \\
\hline Amish & 303.0 & 0.92 & $5,947.2$ & 0.13 & 359.1 & 0.08 \\
\hline Assembly of God & 0.0 & 0.02 & 0.0 & \# & 0.0 & \# \\
\hline Baptist & 70.1 & 0.21 & 664.3 & 0.03 & 79.3 & 0.03 \\
\hline Brethren & 0.0 & \# & 0.0 & \# & 0.0 & \\
\hline Calvinist & 4.8 & 0.02 & 272.0 & 0.01 & 32.9 & 0.01 \\
\hline Christian (unspecified) & 153.2 & 0.32 & $3,655.7$ & 0.09 & 746.7 & 0.14 \\
\hline Church of Christ & 1.1 & 0.01 & 17.2 & \# & 2.2 & \\
\hline Church of God & 1.0 & 0.01 & 1.0 & \# & 1.0 & \\
\hline Church of God in Christ & 0.0 & \# & 0.0 & \# & 0.0 & \\
\hline Church of the Nazarene & 0.0 & \# & 0.0 & \# & 0.0 & \\
\hline Disciples of Christ & $\dagger$ & $\dagger$ & $\dagger$ & $\dagger$ & $\dagger$ & \\
\hline Episcopal & 0.0 & 0.02 & 0.0 & 0.01 & 0.0 & 0.01 \\
\hline Friends & 1.1 & 0.01 & 64.2 & \# & 15.2 & \\
\hline Greek Orthodox & 1.8 & 0.01 & 131.9 & \# & 18.1 & \# \\
\hline Islamic & 1.5 & 0.01 & 14.5 & \# & 2.9 & \\
\hline Jewish & 25.8 & 0.10 & $16,799.1$ & 0.35 & 527.0 & 0.12 \\
\hline Latter Day Saints & $\dagger$ & $\dagger$ & $\dagger$ & $\dagger$ & $\dagger$ & \\
\hline Lutheran Church-Missouri Synod & 3.8 & 0.06 & 832.0 & 0.02 & 49.1 & 0.01 \\
\hline Evangelical Lutheran Church In America & 0.0 & 0.01 & 0.0 & \# & 0.0 & $H$ \\
\hline Wisconsin Evangelical Lutheran Synod & 0.0 & 0.02 & 0.0 & \# & 0.0 & 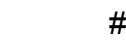 \\
\hline Other Lutheran & 1.0 & 0.01 & 16.0 & \# & 1.9 & \\
\hline Mennonite & 142.7 & 0.43 & $5,217.0$ & 0.11 & 494.5 & 0.12 \\
\hline Methodist & 0.0 & 0.01 & 0.0 & \# & 0.0 & \\
\hline Pentecostal & 9.9 & 0.04 & 82.5 & \# & 19.1 & \\
\hline Presbyterian & 1.0 & 0.01 & 16.5 & \# & 0.6 & \\
\hline Seventh-Day Adventist & 0.0 & 0.05 & 0.0 & 0.01 & 0.0 & \# \\
\hline Other & 0.0 & 0.01 & 0.0 & \# & 0.0 & \# \\
\hline Nonsectarian & 87.1 & 0.63 & $4,512.6$ & 0.15 & 567.0 & 0.17 \\
\hline
\end{tabular}

† Not applicable.

\# Rounds to zero.

SOURCE: U.S. Department of Education, National Center for Education Statistics, Private School Universe Survey

(PSS), 2011-2012. 
Table C-3. Standard errors for percentage distribution of private schools, by program emphasis and selected school characteristics: United States, 2011-12

\begin{tabular}{|c|c|c|c|c|c|c|c|}
\hline $\begin{array}{l}\text { School } \\
\text { characteristic }\end{array}$ & $\begin{array}{r}\text { Regular } \\
\text { elementaryl } \\
\text { secondary }\end{array}$ & Montessori & $\begin{array}{r}\text { Special } \\
\text { program } \\
\text { emphasis }\end{array}$ & $\begin{array}{r}\text { Special } \\
\text { education }\end{array}$ & $\begin{array}{r}\text { Vocational/ } \\
\text { technical } \\
\text { programs } \\
\end{array}$ & Alternative & $\begin{array}{r}\text { Early } \\
\text { childhood } \\
\end{array}$ \\
\hline & 0.31 & 0.23 & 0.17 & 0.15 & 0.04 & 0.14 & 0.04 \\
\hline Total & 0.55 & 0.15 & 0.04 & 0.14 & $\dagger$ & 0.05 & 0.24 \\
\hline \multicolumn{8}{|l|}{ Private school typology } \\
\hline Catholic & 0.20 & \# & \# & 0.12 & $\dagger$ & $\dagger$ & 0.16 \\
\hline Parochial & 0.17 & $\dagger$ & 0.00 & $\dagger$ & $\dagger$ & 0.00 & $t$ \\
\hline Diocesan & 0.23 & $\dagger$ & $\dagger$ & 0.23 & $\dagger$ & $\dagger$ & 0.04 \\
\hline Private & 1.00 & 0.06 & $\dagger$ & 0.06 & $\dagger$ & $\dagger$ & 1.01 \\
\hline Other religious & 0.52 & 0.04 & 0.06 & 0.10 & $\dagger$ & 0.06 & 0.32 \\
\hline Conservative Christian & 0.21 & $\dagger$ & 0.03 & 0.02 & $\dagger$ & 0.05 & 0.10 \\
\hline Other affiliated & 0.23 & \# & 0.01 & 0.24 & $\dagger$ & \# & 0.14 \\
\hline Unaffiliated & 1.25 & 0.12 & 0.10 & 0.14 & $\dagger$ & 0.12 & 0.84 \\
\hline Nonsectarian & 0.48 & 0.18 & 0.06 & 0.23 & $\dagger$ & 0.05 & 0.29 \\
\hline Regular & 0.70 & $\dagger$ & $\dagger$ & $\dagger$ & $\dagger$ & $\dagger$ & 0.70 \\
\hline Special emphasis & $\dagger$ & 0.19 & 0.16 & $\dagger$ & $\dagger$ & 0.12 & $\dagger$ \\
\hline Special education & $\dagger$ & $\dagger$ & $\dagger$ & 0.00 & $\dagger$ & $\dagger$ & $\dagger$ \\
\hline \multicolumn{8}{|l|}{ School level } \\
\hline Elementary & 0.74 & 0.25 & 0.05 & 0.11 & $\dagger$ & 0.03 & 0.41 \\
\hline Secondary & 0.31 & $\dagger$ & 0.03 & 0.21 & $\dagger$ & 0.12 & $\dagger$ \\
\hline Combined & 0.53 & 0.03 & 0.06 & 0.37 & $\dagger$ & 0.08 & 0.01 \\
\hline \multicolumn{8}{|l|}{ Size (number of students) } \\
\hline Less than 50 & 2.08 & 0.54 & 0.09 & 0.35 & $\dagger$ & 0.16 & 1.03 \\
\hline $50-149$ & 0.34 & 0.17 & 0.05 & 0.17 & $\dagger$ & 0.04 & 0.05 \\
\hline 150-299 & 0.03 & 0.02 & 0.01 & 0.01 & $\dagger$ & \# & $\dagger$ \\
\hline $300-499$ & 0.01 & $\dagger$ & 0.01 & \# & $\dagger$ & $\dagger$ & $\dagger$ \\
\hline $500-749$ & 0.05 & 0.00 & $\dagger$ & $\dagger$ & $\dagger$ & $\dagger$ & $\dagger$ \\
\hline 750 or more & 0.02 & 0.00 & $\dagger$ & $\dagger$ & $\dagger$ & $\dagger$ & $\dagger$ \\
\hline \multicolumn{8}{|l|}{ Region } \\
\hline Northeast & 0.47 & 0.17 & 0.07 & 0.23 & $\dagger$ & 0.02 & 0.34 \\
\hline Midwest & 1.36 & 0.46 & 0.09 & 0.34 & $\dagger$ & 0.09 & 0.61 \\
\hline South & 0.26 & 0.07 & 0.03 & 0.05 & $\dagger$ & 0.03 & 0.11 \\
\hline West & 0.05 & 0.02 & 0.03 & 0.02 & $\dagger$ & 0.02 & 0.04 \\
\hline \multicolumn{8}{|l|}{ Urbanicity type } \\
\hline City & 0.23 & 0.06 & 0.04 & 0.20 & $\dagger$ & 0.02 & 0.09 \\
\hline Suburban & 0.28 & 0.12 & 0.05 & 0.16 & $\dagger$ & 0.02 & 0.30 \\
\hline Town & 0.58 & 0.18 & 0.05 & 0.08 & $\dagger$ & 0.07 & 0.20 \\
\hline Rural & 1.21 & 0.24 & 0.12 & 0.28 & $\dagger$ & 0.23 & 0.40 \\
\hline
\end{tabular}

† Not applicable.

\# Rounds to zero.

SOURCE: U.S. Department of Education, National Center for Education Statistics, Private School Universe Survey (PSS), 2011-2012. 
Table C-4. Standard errors for number and percentage distribution of private schools, by urbanicity type and selected school characteristics: United States, 2011-12

\begin{tabular}{|c|c|c|c|c|c|c|c|c|c|c|}
\hline \multirow{2}{*}{$\begin{array}{l}\text { School } \\
\text { characteristic }\end{array}$} & \multicolumn{2}{|c|}{ Total } & \multicolumn{2}{|c|}{ City } & \multicolumn{2}{|c|}{ Suburban } & \multicolumn{2}{|c|}{ Town } & \multicolumn{2}{|c|}{ Rural } \\
\hline & \multicolumn{2}{|c|}{$\overline{\text { Number Percent }}$} & \multicolumn{2}{|c|}{ Number Percent } & \multicolumn{2}{|c|}{ Number Percent } & \multicolumn{2}{|c|}{ Number Percent } & \multicolumn{2}{|c|}{ Number Percent } \\
\hline & 264.4 & 0.62 & 104.4 & 0.35 & 49.0 & 0.26 & & & & \\
\hline Total & 542.4 & $\dagger$ & 51.7 & $\dagger$ & 69.7 & $\dagger$ & 103.7 & $\dagger$ & 452.8 & $\dagger$ \\
\hline \multicolumn{11}{|l|}{ Private school typology } \\
\hline Catholic & 31.1 & 0.41 & 27.1 & 0.23 & 27.4 & 0.24 & 1.0 & 1.16 & 0.0 & 0.56 \\
\hline Parochial & 15.7 & 0.17 & 2.4 & 0.06 & 15.5 & 0.14 & 1.0 & 0.57 & 0.0 & 0.23 \\
\hline Diocesan & 27.8 & 0.19 & 27.0 & 0.24 & 6.9 & 0.09 & 0.0 & 0.53 & 0.0 & 0.22 \\
\hline Private & 17.5 & 0.08 & 1.3 & 0.03 & 17.4 & 0.15 & 0.0 & 0.06 & 0.0 & 0.11 \\
\hline Other religious & 550.9 & 0.99 & 9.1 & 0.19 & 23.5 & 0.19 & 103.7 & 1.69 & 463.3 & 2.18 \\
\hline Conservative Christian & 127.3 & 0.27 & 1.0 & 0.06 & 9.8 & 0.07 & 88.8 & 2.39 & 90.7 & 1.53 \\
\hline Other affiliated & 17.1 & 0.18 & 6.6 & 0.08 & 15.3 & 0.13 & 0.0 & 0.34 & 4.0 & 0.55 \\
\hline Unaffiliated & 458.3 & 1.12 & 4.8 & 0.08 & 10.8 & 0.11 & 53.6 & 1.60 & 444.1 & 3.70 \\
\hline Nonsectarian & 87.1 & 0.63 & 41.2 & 0.28 & 56.4 & 0.34 & 1.0 & 0.54 & 45.7 & 1.66 \\
\hline Regular & 71.1 & 0.37 & 32.7 & 0.28 & 44.2 & 0.32 & 0.0 & 0.23 & 45.3 & 1.05 \\
\hline Special emphasis & 23.2 & 0.19 & 8.7 & 0.08 & 19.3 & 0.14 & 1.0 & 0.24 & 4.6 & 0.46 \\
\hline Special education & 21.9 & 0.11 & 21.0 & 0.20 & 5.0 & 0.05 & 0.0 & 0.07 & 4.1 & 0.25 \\
\hline \multicolumn{11}{|l|}{ School level } \\
\hline Elementary & 414.1 & 0.43 & 40.6 & 0.24 & 60.9 & 0.31 & 1.8 & 2.15 & 406.7 & 2.68 \\
\hline Secondary & 30.1 & 0.18 & 28.7 & 0.26 & 11.8 & 0.11 & 0.0 & 0.22 & 4.9 & 0.49 \\
\hline Combined & 188.3 & 0.38 & 9.7 & 0.13 & 33.6 & 0.27 & 103.7 & 2.36 & 149.8 & 2.37 \\
\hline \multicolumn{11}{|l|}{ Program emphasis } \\
\hline Regular elementary/ & & & & & & & & & & \\
\hline secondary & 539.9 & 0.55 & 42.9 & 0.23 & 42.2 & 0.28 & 103.7 & 0.58 & 452.5 & 1.21 \\
\hline Montessori & 19.6 & 0.15 & 6.0 & 0.06 & 17.0 & 0.12 & 1.0 & 0.18 & 4.6 & 0.24 \\
\hline Special program emphasis & 7.5 & 0.04 & 4.7 & 0.04 & 5.8 & 0.05 & 0.0 & 0.05 & 1.1 & 0.12 \\
\hline Special education & 28.0 & 0.14 & 21.0 & 0.20 & 18.1 & 0.16 & 0.0 & 0.08 & 4.1 & 0.28 \\
\hline Vocational/technical & $\dagger$ & $\dagger$ & $\dagger$ & $\dagger$ & $\dagger$ & $\dagger$ & $\dagger$ & $\dagger$ & $\dagger$ & $\dagger$ \\
\hline Alternative & 1.9 & 0.05 & 1.4 & 0.02 & 1.2 & 0.02 & 0.0 & 0.07 & 0.0 & 0.23 \\
\hline Early childhood & 43.8 & 0.24 & 8.8 & 0.09 & 38.7 & 0.30 & 0.0 & 0.20 & 16.1 & 0.40 \\
\hline \multicolumn{11}{|l|}{ Size (number of students) } \\
\hline Less than 50 & 521.2 & 0.95 & 40.1 & 0.28 & 47.0 & 0.23 & 88.9 & 1.99 & 443.8 & 2.57 \\
\hline $50-149$ & 114.1 & 0.48 & 5.7 & 0.12 & 32.9 & 0.23 & 53.5 & 1.63 & 58.0 & 1.61 \\
\hline 150-299 & 14.7 & 0.32 & 11.6 & 0.14 & 9.1 & 0.12 & 0.0 & 0.57 & 0.0 & 0.66 \\
\hline $300-499$ & 7.0 & 0.14 & 1.0 & 0.05 & 6.9 & 0.07 & 0.0 & 0.17 & 0.0 & 0.23 \\
\hline $500-749$ & 27.0 & 0.11 & 27.0 & 0.26 & 0.0 & 0.03 & 0.0 & 0.04 & 0.0 & 0.09 \\
\hline 750 or more & 9.9 & 0.05 & 0.0 & 0.02 & 0.0 & 0.01 & $\dagger$ & $\dagger$ & 9.9 & 0.15 \\
\hline \multicolumn{11}{|l|}{ Region } \\
\hline Northeast & 67.8 & 0.45 & 11.9 & 0.13 & 39.4 & 0.30 & 3.8 & 0.45 & 42.7 & 1.64 \\
\hline Midwest & 532.7 & 1.29 & 46.2 & 0.37 & 57.2 & 0.42 & 103.6 & 2.12 & 444.8 & 4.14 \\
\hline South & 76.4 & 0.55 & 19.6 & 0.20 & 4.7 & 0.18 & 1.4 & 1.04 & 73.3 & 2.04 \\
\hline West & 6.7 & 0.36 & 3.4 & 0.15 & 3.8 & 0.13 & 1.5 & 0.65 & 2.9 & 0.68 \\
\hline
\end{tabular}

† Not applicable.

SOURCE: U.S. Department of Education, National Center for Education Statistics, Private School Universe Survey (PSS), 2011-2012. 
Table C-5. Standard errors for number and percentage distribution of private school students, by urbanicity type and selected school characteristics: United States, 2011-12

\begin{tabular}{|c|c|c|c|c|c|c|c|c|c|c|}
\hline \multirow{2}{*}{$\begin{array}{l}\begin{array}{l}\text { School } \\
\text { characteristic }\end{array} \\
\frac{\text { Total }}{}\end{array}$} & \multicolumn{2}{|c|}{$\begin{array}{c}\text { Total } \\
\text { Number Percent }\end{array}$} & \multicolumn{2}{|c|}{$\begin{array}{c}\text { City } \\
\text { Number Percent }\end{array}$} & \multicolumn{2}{|c|}{$\begin{array}{c}\text { Suburban } \\
\text { Number Percent }\end{array}$} & \multicolumn{2}{|c|}{$\begin{array}{c}\text { Town } \\
\text { Number Percent }\end{array}$} & \multicolumn{2}{|c|}{$\begin{array}{c}\text { Rural } \\
\text { Number Percen }\end{array}$} \\
\hline & $25,138.6$ & $\dagger$ & $14,550.9$ & $\dagger$ & $5,325.6$ & $\dagger$ & $3,128.6$ & $\dagger$ & $19,863.7$ & $\dagger$ \\
\hline \multicolumn{11}{|l|}{ Private school typology } \\
\hline Catholic & $14,630.3$ & 0.28 & $14,317.6$ & 0.40 & $3,476.2$ & 0.13 & 103.7 & 0.54 & 0.0 & 0.55 \\
\hline Parochial & $3,325.6$ & 0.11 & 397.4 & 0.12 & $3,300.2$ & 0.15 & 103.7 & 0.26 & 0.0 & 0.20 \\
\hline Diocesan & $14,320.0$ & 0.28 & $14,312.1$ & 0.60 & 474.1 & 0.07 & 0.0 & 0.26 & 0.0 & 0.24 \\
\hline Private & 217.6 & 0.05 & 29.0 & 0.08 & 215.6 & 0.02 & 0.0 & 0.02 & 0.0 & 0.11 \\
\hline Other religious & $21,908.0$ & 0.34 & $1,341.1$ & 0.25 & $2,195.0$ & 0.10 & $3,126.8$ & 0.65 & $21,021.5$ & 1.57 \\
\hline Conservative Christian & $4,641.5$ & 0.11 & 17.8 & 0.08 & 564.8 & 0.05 & $1,421.9$ & 0.42 & $4,381.8$ & 0.86 \\
\hline Other affiliated & $2,397.8$ & 0.09 & $1,290.1$ & 0.10 & $1,982.7$ & 0.10 & 0.0 & 0.09 & 392.6 & 0.32 \\
\hline Unaffiliated & $20,414.6$ & 0.40 & 180.4 & 0.09 & 417.1 & 0.04 & $2,784.8$ & 0.81 & $19,524.4$ & 2.36 \\
\hline Nonsectarian & $4,512.6$ & 0.15 & $2,051.0$ & 0.17 & $2,430.9$ & 0.13 & 12.0 & 0.11 & $3,211.8$ & 1.07 \\
\hline Regular & $4,303.4$ & 0.13 & $1,894.5$ & 0.14 & $2,194.5$ & 0.12 & 0.0 & 0.08 & $3,186.5$ & 0.88 \\
\hline Special emphasis & 870.4 & 0.02 & 499.2 & 0.04 & 695.9 & 0.04 & 12.0 & 0.03 & 45.5 & 0.14 \\
\hline Special education & 693.6 & 0.02 & 549.4 & 0.03 & 139.7 & 0.01 & 0.0 & 0.01 & 399.7 & 0.10 \\
\hline \multicolumn{11}{|l|}{ School level } \\
\hline Elementary & $8,772.0$ & 0.24 & $1,268.6$ & 0.36 & $4,400.4$ & 0.15 & 119.6 & 0.55 & $7,460.2$ & 1.22 \\
\hline Secondary & $14,401.0$ & 0.28 & $14,428.3$ & 0.60 & 326.8 & 0.05 & 0.0 & 0.09 & 272.0 & 0.40 \\
\hline Combined & $17,100.8$ & 0.26 & $1,336.2$ & 0.25 & $2,726.1$ & 0.14 & $3,126.3$ & 0.64 & $18,082.7$ & 1.43 \\
\hline \multicolumn{11}{|l|}{ Program emphasis } \\
\hline \multicolumn{11}{|l|}{ Regular elementary/ } \\
\hline secondary & $25,216.0$ & 0.06 & $14,523.2$ & 0.07 & $5,096.8$ & 0.04 & $3,128.5$ & 0.05 & $19,857.9$ & 0.31 \\
\hline Montessori & 781.7 & 0.02 & 450.2 & 0.03 & 633.9 & 0.04 & 12.0 & 0.01 & 45.5 & 0.05 \\
\hline Special program emphasis & 240.2 & 0.01 & 71.9 & 0.01 & 228.7 & 0.01 & 0.0 & 0.01 & 16.2 & 0.07 \\
\hline Special education & 943.4 & 0.03 & 553.1 & 0.03 & 651.4 & 0.04 & 0.0 & 0.01 & 399.7 & 0.10 \\
\hline Vocational/technical & $\dagger$ & $\dagger$ & $\dagger$ & $\dagger$ & $\dagger$ & $\dagger$ & $\dagger$ & $\dagger$ & $\dagger$ & 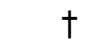 \\
\hline Alternative & 112.8 & 0.01 & 102.9 & 0.01 & 46.1 & \# & 0.0 & 0.01 & 0.0 & 0.06 \\
\hline Early childhood & 627.9 & 0.02 & 120.3 & 0.01 & 489.1 & 0.03 & 0.0 & 0.01 & 256.6 & 0.06 \\
\hline \multicolumn{11}{|l|}{ Size (number of students) } \\
\hline Less than 50 & $7,510.8$ & 0.16 & 789.3 & 0.05 & 786.3 & 0.04 & $1,431.7$ & 0.44 & $6,282.3$ & 0.94 \\
\hline 50-149 & $7,353.2$ & 0.16 & 512.8 & 0.09 & $2,240.3$ & 0.12 & $2,781.8$ & 0.63 & $4,337.8$ & 0.86 \\
\hline 150-299 & $2,944.8$ & 0.17 & $2,306.3$ & 0.21 & $1,831.1$ & 0.06 & 0.0 & 0.33 & 0.0 & 0.79 \\
\hline $300-499$ & $2,848.3$ & 0.12 & 395.2 & 0.16 & $2,820.7$ & 0.11 & 0.0 & 0.18 & 0.0 & 0.50 \\
\hline $500-749$ & $14,312.1$ & 0.28 & $14,312.1$ & 0.63 & 0.0 & 0.05 & 0.0 & 0.07 & 0.0 & 0.31 \\
\hline 750 or more & $17,907.6$ & 0.34 & 0.0 & 0.17 & 0.0 & 0.05 & $\dagger$ & $\dagger$ & $17,907.6$ & 2.56 \\
\hline \multicolumn{11}{|l|}{ Region } \\
\hline Northeast & $17,327.1$ & 0.31 & $1,551.4$ & 0.17 & $2,347.8$ & 0.13 & 176.4 & 0.12 & $18,017.7$ & 2.29 \\
\hline Midwest & $18,087.1$ & 0.32 & $14,338.0$ & 0.59 & $4,765.1$ & 0.22 & $3,121.3$ & 0.57 & $8,276.9$ & 1.23 \\
\hline South & $2,335.7$ & 0.19 & $1,934.5$ & 0.26 & 318.8 & 0.09 & 59.6 & 0.33 & $1,179.1$ & 1.37 \\
\hline West & 302.9 & 0.11 & 38.5 & 0.18 & 204.7 & 0.05 & 104.6 & 0.13 & 176.8 & 0.36 \\
\hline
\end{tabular}

† Not applicable.

\# Rounds to zero.

SOURCE: U.S. Department of Education, National Center for Education Statistics, Private School Universe Survey (PSS),

2011-2012. 
Table C-6. Standard errors for number and percentage distribution of private school students, by grade and private school typology: United States, 2011-12

\begin{tabular}{|c|c|c|c|c|c|c|c|c|c|c|}
\hline \multirow{3}{*}{$\frac{\text { Private school typology }}{\text { Total }}$} & \multirow{2}{*}{\multicolumn{2}{|c|}{$\begin{array}{c}\text { Kindergarten } \\
\text { Number Percent }\end{array}$}} & \multirow{2}{*}{\multicolumn{2}{|c|}{$\begin{array}{c}\text { First grade } \\
\text { Number Percent }\end{array}$}} & \multirow{2}{*}{\multicolumn{2}{|c|}{$\begin{array}{c}\text { Second grade } \\
\text { Number Percent }\end{array}$}} & \multirow{2}{*}{\multicolumn{2}{|c|}{$\begin{array}{c}\text { Third grade } \\
\text { Number Percent }\end{array}$}} & \multicolumn{2}{|c|}{ Fourth grade } \\
\hline & & & & & & & & & \multicolumn{2}{|c|}{ Number Percent } \\
\hline & $2,989.3$ & $\dagger$ & $2,191.0$ & $\dagger$ & $2,008.1$ & $\dagger$ & $1,850.1$ & $\dagger$ & $1,921.5$ & $\dagger$ \\
\hline Catholic & 451.0 & 0.22 & 429.0 & 0.26 & 427.9 & 0.25 & 349.7 & 0.24 & 360.6 & 0.25 \\
\hline Parochial & 385.4 & 0.13 & 405.4 & 0.15 & 396.2 & 0.15 & 325.4 & 0.13 & 354.5 & 0.14 \\
\hline Diocesan & 13.2 & 0.10 & 47.4 & 0.12 & 27.1 & & & & 27.1 & 0.11 \\
\hline Private & 122.9 & 0.03 & 27.3 & 0.02 & 31.0 & 0.02 & 72.8 & 0.03 & 5.2 & 0.02 \\
\hline Other religious & $2,497.8$ & 0.30 & $2,067.0$ & 0.34 & $1,876.7$ & 0.32 & $1,751.2$ & 0.31 & $1,828.7$ & 0.33 \\
\hline Conservative Christian & 447.3 & 0.11 & 352.8 & 0.09 & 427.9 & 0.08 & & 0.11 & 389.9 & 0.10 \\
\hline Other affiliated & 370.5 & 0.12 & 246.8 & 0.10 & 267.5 & 0.10 & 228.7 & 0.09 & 271.2 & 0.10 \\
\hline Unaffiliated & $2,519.2$ & 0.47 & $1,932.5$ & 0.47 & $1,577.5$ & 0.39 & $1,497.3$ & 0.38 & $1,672.6$ & 0.44 \\
\hline Nonsectarian & 737.4 & 0.14 & 191.0 & 0.10 & 191.5 & 0.09 & 202.0 & 0.09 & 241.6 & 0.11 \\
\hline Regular & 570.4 & 0.11 & 134.4 & 0.08 & 142.2 & 0.08 & 171.1 & 0.08 & 230.5 & 0.09 \\
\hline Special emphasis & 277.8 & 0.06 & 117.7 & 0.03 & 127.1 & 0.03 & 105.1 & 0.03 & 60.1 & 0.02 \\
\hline \multirow[t]{2}{*}{ Special education } & 20.2 & 0.01 & 0.0 & $\#$ & 0.0 & \# & 1.0 & 0.01 & 4.2 & 0.01 \\
\hline & \multicolumn{2}{|c|}{ Fifth grade } & \multicolumn{2}{|c|}{ Sixth grade } & \multicolumn{2}{|c|}{ Seventh grade } & \multicolumn{2}{|c|}{ Eighth grade } & \multicolumn{2}{|c|}{ Ninth grade } \\
\hline Private school typology & \multicolumn{2}{|c|}{$\overline{\text { Number Percent }}$} & \multicolumn{2}{|c|}{$\begin{array}{l}\text { Number Percent } \\
\end{array}$} & \multicolumn{2}{|c|}{ Number Percent } & \multicolumn{2}{|c|}{$\overline{\text { Number Percent }}$} & \multicolumn{2}{|c|}{$\begin{array}{l}\text { Number Percent } \\
\end{array}$} \\
\hline Total & $1,832.1$ & $\dagger$ & $1,766.4$ & $\dagger$ & $1,684.1$ & $\dagger$ & $1,950.9$ & $\dagger$ & $3,875.3$ & $\dagger$ \\
\hline Catholic & 402.8 & 0.25 & 394.6 & 0.24 & 357.5 & 0.23 & 324.4 & 0.26 & $3,612.0$ & 0.61 \\
\hline Parochial & 395.6 & 0.15 & 381.5 & 0.14 & 349.7 & 0.13 & 322.8 & 0.14 & 5.2 & 0.06 \\
\hline Diocesan & 20.3 & 0.11 & 47.4 & 0.11 & 74.5 & 0.11 & 27.1 & 0.11 & $3,612.0$ & 0.87 \\
\hline Private & 35.9 & 0.02 & 10.3 & 0.02 & 0.0 & 0.02 & 17.8 & 0.03 & 0.0 & 0.25 \\
\hline Other religious & $1,832.0$ & 0.34 & $1,742.7$ & 0.32 & $1,680.9$ & 0.32 & $2,047.9$ & 0.40 & $1,464.9$ & 0.49 \\
\hline Conservative Christian & 385.7 & 0.08 & 291.1 & 0.07 & 518.4 & 0.13 & 401.8 & 0.11 & 414.6 & 0.19 \\
\hline Other affiliated & 193.3 & 0.09 & 184.0 & 0.09 & 135.6 & 0.08 & 207.1 & 0.10 & 145.0 & 0.13 \\
\hline Unaffiliated & $1,586.5$ & 0.41 & $1,624.5$ & 0.41 & $1,487.3$ & 0.38 & $1,913.2$ & 0.50 & $1,466.2$ & 0.42 \\
\hline Nonsectarian & 176.5 & 0.11 & 179.5 & 0.10 & 249.7 & 0.11 & 280.5 & 0.15 & 860.4 & 0.33 \\
\hline Regular & 136.1 & 0.09 & 153.8 & 0.08 & 248.0 & 0.10 & 283.2 & 0.13 & 850.3 & 0.30 \\
\hline Special emphasis & 81.3 & 0.03 & 66.0 & 0.02 & 39.0 & 0.01 & 18.8 & 0.02 & 115.1 & 0.04 \\
\hline Special education & 13.1 & 0.01 & 36.4 & 0.01 & 16.6 & 0.01 & 44.1 & 0.02 & 64.0 & 0.04 \\
\hline
\end{tabular}

\section{Tenth grade Eleventh grade Twelfth grade Ungraded}

Private school typology Number Percent $\quad$ Number Percent $\quad$ Number Percent

$\begin{array}{lrrrrrrrr}\text { Total } & 4,160.6 & \dagger & 3,647.1 & \dagger & 3,768.5 & \dagger & 689.5 & \dagger \\ \text { Catholic } & 3,908.7 & 0.68 & 3,288.6 & 0.60 & 3,503.9 & 0.62 & 0.0 & 0.08 \\ \text { Parochial } & 5.2 & 0.07 & 0.0 & 0.06 & 0.0 & 0.06 & 0.0 & 0.02 \\ \text { Diocesan } & 3,908.7 & 0.96 & 3,288.6 & 0.83 & 3,503.9 & 0.89 & 0.0 & 0.03 \\ \text { Private } & 0.0 & 0.27 & 0.0 & 0.24 & 0.0 & 0.25 & 0.0 & 0.03 \\ \text { Other religious } & 1,578.2 & 0.54 & 1,445.2 & 0.48 & 1,314.2 & 0.46 & 417.3 & 0.63 \\ \text { Conservative Christian } & 463.0 & 0.22 & 281.6 & 0.18 & 207.3 & 0.16 & 88.8 & 0.15 \\ \text { Other affiliated } & 112.4 & 0.13 & 129.0 & 0.12 & 134.6 & 0.13 & 322.6 & 0.53 \\ \text { Unaffiliated } & 1,489.9 & 0.44 & 1,482.6 & 0.44 & 1,389.0 & 0.42 & 216.6 & 0.35 \\ \text { Nonsectarian } & 929.8 & 0.38 & 1,130.2 & 0.39 & 696.6 & 0.32 & 548.9 & 0.60 \\ \quad \text { Regular } & 920.5 & 0.34 & 1,122.0 & 0.36 & 688.5 & 0.27 & 0.0 & 0.12 \\ \text { Special emphasis } & 97.3 & 0.04 & 106.5 & 0.04 & 92.1 & 0.04 & 49.7 & 0.25 \\ \text { Special education } & 87.9 & 0.04 & 84.0 & 0.04 & 52.0 & 0.04 & 546.7 & 0.59\end{array}$

† Not applicable.

\# Rounds to zero.

SOURCE: U.S. Department of Education, National Center for Education Statistics, Private School Universe Survey (PSS), 2011-2012. 
Table C-7. Standard errors for average private school size, by school level and selected school characteristics: United States, 2011-12

\begin{tabular}{|c|c|c|c|c|}
\hline \multirow{2}{*}{$\begin{array}{l}\text { School } \\
\text { characteristic }\end{array}$} & \multicolumn{4}{|c|}{ Average number of students } \\
\hline & Total & Elementary & Secondary & Combined \\
\hline Total & 2.38 & 1.94 & 2.77 & 4.32 \\
\hline \multicolumn{5}{|l|}{ Private school typology } \\
\hline Catholic & 1.52 & 0.67 & 0.12 & 6.12 \\
\hline Parochial & 0.22 & 0.31 & 0.00 & 22.61 \\
\hline Diocesan & 2.33 & 0.10 & 0.17 & 13.70 \\
\hline Private & 5.77 & 5.69 & 0.00 & 0.00 \\
\hline Other religious & 4.04 & 3.06 & 1.87 & 6.39 \\
\hline Conservative Christian & 3.14 & 3.48 & 0.00 & 4.94 \\
\hline Other affiliated & 0.62 & 0.33 & 0.00 & 2.26 \\
\hline Unaffiliated & 5.59 & 3.68 & 3.69 & 15.00 \\
\hline Nonsectarian & 0.52 & 0.30 & 1.05 & 2.56 \\
\hline Regular & 1.22 & 0.50 & 0.82 & 14.80 \\
\hline Special emphasis & 0.21 & 0.18 & 0.50 & 0.33 \\
\hline Special education & 0.53 & 1.75 & 0.00 & 0.40 \\
\hline \multicolumn{5}{|l|}{ Program emphasis } \\
\hline \multicolumn{5}{|l|}{ Regular elementary/ } \\
\hline secondary & 4.46 & 4.24 & 2.52 & 6.36 \\
\hline Montessori & 0.21 & 0.19 & $\dagger$ & 0.00 \\
\hline Special program emphasis & 1.07 & 1.19 & 0.00 & 1.06 \\
\hline Special education & 0.55 & 1.39 & 0.54 & 0.55 \\
\hline Vocational/technical & $t$ & $\dagger$ & $\dagger$ & $\dagger$ \\
\hline Alternative & 0.07 & 0.23 & 0.02 & 0.00 \\
\hline Early childhood & 0.08 & 0.08 & $\dagger$ & 0.00 \\
\hline \multicolumn{5}{|l|}{ Size (number of students) } \\
\hline Less than 50 & 0.28 & 0.24 & 0.08 & 0.85 \\
\hline $50-149$ & 0.41 & 0.25 & 0.31 & 0.79 \\
\hline 150-299 & 0.05 & 0.06 & 0.14 & 0.10 \\
\hline $300-499$ & 0.12 & 0.22 & 0.00 & 0.14 \\
\hline $500-749$ & 1.76 & 0.00 & 6.51 & 0.00 \\
\hline 750 or more & 10.25 & 0.00 & 0.00 & 17.13 \\
\hline \multicolumn{5}{|l|}{ Region } \\
\hline Northeast & 2.16 & 0.77 & 3.56 & 10.14 \\
\hline Midwest & 8.60 & 7.17 & 8.45 & 14.57 \\
\hline South & 1.17 & 1.40 & 0.84 & 0.21 \\
\hline West & 0.11 & 0.09 & 0.43 & 0.29 \\
\hline \multicolumn{5}{|l|}{ Urbanicity type } \\
\hline City & 1.16 & 0.70 & 4.63 & 0.72 \\
\hline Suburban & 0.72 & 0.83 & 3.78 & 1.91 \\
\hline Town & 2.86 & 0.05 & 0.00 & 9.91 \\
\hline Rural & 5.38 & 3.83 & 0.81 & 9.52 \\
\hline
\end{tabular}

† Not applicable.

SOURCE: U.S. Department of Education, National Center for Education Statistics, Private School Universe Survey (PSS), 2011-2012. 
Table C-8. Standard errors for number and percentage distribution of private schools, by school size and selected school characteristics: United States, 2011-12

\begin{tabular}{|c|c|c|c|c|c|c|c|c|c|c|c|c|}
\hline \multirow{3}{*}{$\begin{array}{l}\begin{array}{l}\text { School } \\
\text { characteristic }\end{array} \\
\text { Total }\end{array}$} & \multirow{2}{*}{\multicolumn{2}{|c|}{$\begin{array}{c}\begin{array}{c}\text { Less than } 50 \\
\text { students }\end{array} \\
\text { NumberPercent }\end{array}$}} & \multirow{2}{*}{\multicolumn{2}{|c|}{$\begin{array}{c}50-149 \\
\text { students } \\
\text { NumberPercent }\end{array}$}} & \multirow{2}{*}{\multicolumn{2}{|c|}{$\begin{array}{c}150-299 \\
\text { students } \\
\text { NumberPercent }\end{array}$}} & \multirow{2}{*}{\multicolumn{2}{|c|}{$\begin{array}{c}\text { 300-499 } \\
\text { students } \\
\text { NumberPercent }\end{array}$}} & \multirow{2}{*}{\multicolumn{2}{|c|}{$\begin{array}{c}500-749 \\
\text { students } \\
\text { NumberPercent }\end{array}$}} & \multirow{2}{*}{\multicolumn{2}{|c|}{$\begin{array}{c}750 \text { or more } \\
\text { students }\end{array}$}} \\
\hline & & & & & & & & & & & & \\
\hline & 521.2 & 0.95 & 114.1 & 0.48 & 14.7 & 0.32 & 7.0 & 0.14 & 27.0 & 0.11 & 9.9 & 0.05 \\
\hline \multicolumn{13}{|l|}{ Private school typology } \\
\hline Catholic & 19.7 & 0.28 & 6.8 & 0.13 & 5.7 & 0.14 & 5.2 & 0.07 & 27.0 & 0.37 & 0.0 & 0.02 \\
\hline Parochial & 5.2 & 0.16 & 1.0 & 0.13 & 5.7 & 0.08 & 5.2 & 0.07 & 0.0 & 0.03 & 0.0 & 0.01 \\
\hline Diocesan & 1.1 & 0.05 & 6.8 & 0.28 & 0.0 & 0.38 & 0.0 & 0.18 & 27.0 & 0.83 & 0.0 & 0.04 \\
\hline Private & 17.5 & 1.38 & 0.0 & 0.31 & 0.0 & 0.34 & 0.0 & 0.28 & 0.0 & 0.23 & 0.0 & 0.21 \\
\hline $\begin{array}{r}\text { Other religious } \\
\text { Conservative }\end{array}$ & 517.2 & 1.88 & 151.2 & 1.22 & 7.5 & 0.54 & 4.7 & 0.20 & 0.0 & 0.09 & 9.9 & 0.10 \\
\hline Christian & 115.1 & 1.62 & 54.3 & 1.16 & 0.0 & 0.48 & 0.0 & 0.18 & 0.0 & 0.08 & 0.0 & 0.06 \\
\hline Other affiliated & 8.7 & 0.17 & 7.2 & 0.15 & 7.5 & 0.22 & 4.7 & 0.15 & 0.0 & 0.02 & 0.0 & 0.02 \\
\hline Unaffiliated & 438.4 & 2.56 & 98.2 & 1.79 & 0.0 & 0.64 & 0.0 & 0.23 & 0.0 & 0.09 & 9.9 & 0.17 \\
\hline Nonsectarian & 63.0 & 0.40 & 53.6 & 0.44 & 8.8 & 0.11 & 0.0 & 0.04 & 0.0 & 0.02 & 0.0 & 0.01 \\
\hline Regular & 46.4 & 0.75 & 52.3 & 0.91 & 8.7 & 0.22 & 0.0 & 0.10 & 0.0 & 0.05 & 0.0 & 0.04 \\
\hline Special emphasis & 20.0 & 0.30 & 11.1 & 0.29 & 1.0 & 0.05 & 0.0 & 0.01 & $\dagger$ & $\dagger$ & $\dagger$ & $\dagger$ \\
\hline Special education & 21.6 & 0.63 & 4.0 & 0.52 & 0.0 & 0.10 & 0.0 & 0.02 & $\dagger$ & $\dagger$ & $\dagger$ & $\dagger$ \\
\hline \multicolumn{13}{|l|}{ School level } \\
\hline Elementary & 405.8 & 1.03 & 45.3 & 0.49 & 10.2 & 0.38 & 5.2 & 0.14 & 0.0 & 0.04 & 0.0 & 0.01 \\
\hline Secondary & 11.9 & 0.43 & 6.8 & 0.34 & 8.4 & 0.34 & 0.0 & 0.16 & 27.0 & 0.89 & 0.0 & 0.10 \\
\hline Combined & 172.6 & 1.38 & 75.0 & 0.87 & 6.4 & 0.40 & 4.7 & 0.20 & 0.0 & 0.12 & 9.9 & 0.16 \\
\hline \multicolumn{13}{|l|}{ Program emphasis } \\
\hline \multicolumn{13}{|l|}{ Regular elementary/ } \\
\hline secondary & 518.3 & 1.70 & 113.7 & 0.75 & 14.7 & 0.60 & 7.0 & 0.28 & 27.0 & 0.18 & 9.9 & 0.09 \\
\hline $\begin{array}{l}\text { Montessori } \\
\text { Special program }\end{array}$ & 16.0 & 0.38 & 11.1 & 0.38 & 1.0 & 0.05 & $\dagger$ & $\dagger$ & 0.0 & 0.00 & 0.0 & 0.00 \\
\hline emphasis & 7.4 & 0.69 & 1.0 & 0.40 & 0.0 & 0.18 & 0.0 & 0.09 & $\dagger$ & $\dagger$ & $\dagger$ & $\dagger$ \\
\hline Special education & 26.9 & 0.68 & 7.9 & 0.59 & 0.0 & 0.11 & 0.0 & 0.02 & $\dagger$ & $\dagger$ & $\dagger$ & $\dagger$ \\
\hline Vocational/technical & $\dagger$ & $\dagger$ & $\dagger$ & $\dagger$ & $\dagger$ & $\dagger$ & $\dagger$ & $\dagger$ & $\dagger$ & $\dagger$ & $\dagger$ & $\dagger$ \\
\hline Alternative & 1.5 & 0.11 & 1.0 & 0.11 & 0.0 & 0.01 & $\dagger$ & $\dagger$ & $\dagger$ & $\dagger$ & $\dagger$ & $\dagger$ \\
\hline Early childhood & 42.7 & 0.09 & 3.6 & 0.09 & $\dagger$ & $\dagger$ & $\dagger$ & $\dagger$ & $\dagger$ & $\dagger$ & $\dagger$ & $\dagger$ \\
\hline \multicolumn{13}{|l|}{ Region } \\
\hline Northeast & 49.0 & 0.39 & 38.5 & 0.41 & 8.0 & 0.22 & 0.0 & 0.07 & 0.0 & 0.03 & 9.9 & 0.13 \\
\hline Midwest & 513.5 & 3.80 & 107.3 & 1.97 & 9.0 & 1.15 & 6.9 & 0.53 & 27.0 & 0.45 & 0.0 & 0.11 \\
\hline South & 74.9 & 0.48 & 4.8 & 0.21 & 8.5 & 0.16 & 1.0 & 0.07 & 0.0 & 0.03 & 0.0 & 0.03 \\
\hline West & 5.0 & 0.05 & 3.7 & 0.05 & 0.0 & 0.02 & 0.0 & 0.01 & 0.0 & \# & 0.0 & \# \\
\hline \multicolumn{13}{|l|}{ Urbanicity type } \\
\hline City & 40.1 & 0.28 & 5.7 & 0.12 & 11.6 & 0.14 & 1.0 & 0.05 & 27.0 & 0.26 & 0.0 & 0.02 \\
\hline Suburban & 47.0 & 0.23 & 32.9 & 0.23 & 9.1 & 0.12 & 6.9 & 0.07 & 0.0 & 0.03 & 0.0 & 0.01 \\
\hline Town & 88.9 & 1.99 & 53.5 & 1.63 & 0.0 & 0.57 & 0.0 & 0.17 & 0.0 & 0.04 & $\dagger$ & $\dagger$ \\
\hline Rural & 443.8 & 2.57 & 58.0 & 1.61 & 0.0 & 0.66 & 0.0 & 0.23 & 0.0 & 0.09 & 9.9 & 0.15 \\
\hline
\end{tabular}

† Not applicable.

\# Rounds to zero.

SOURCE: U.S. Department of Education, National Center for Education Statistics, Private School Universe Survey (PSS), 20112012. 
Table C-9. Standard errors for percentage distribution of private school students, by racial/ethnic background and selected school characteristics: United States, 2011-12

\begin{tabular}{|c|c|c|c|c|c|c|c|}
\hline \multirow[b]{2}{*}{$\begin{array}{l}\text { School } \\
\text { characteristic }\end{array}$} & \multirow[b]{2}{*}{ Hispanic } & \multicolumn{6}{|c|}{ Non-Hispanic } \\
\hline & & $\begin{array}{r}\text { American } \\
\text { Indian }\end{array}$ & Asian & Black & $\begin{array}{l}\text { Pacific } \\
\text { Islander }\end{array}$ & White & $\begin{array}{r}\text { Two or more } \\
\text { races }\end{array}$ \\
\hline Total & 0.05 & $\#$ & 0.03 & 0.06 & $\#$ & 0.15 & 0.01 \\
\hline \multicolumn{8}{|l|}{ Private school typology } \\
\hline Catholic & 0.02 & \# & 0.02 & 0.04 & \# & 0.10 & 0.01 \\
\hline Parochial & 0.06 & \# & 0.02 & 0.02 & \# & 0.10 & 0.01 \\
\hline Diocesan & 0.02 & 0.01 & 0.04 & 0.10 & \# & 0.20 & 0.04 \\
\hline Private & 0.01 & \# & \# & 0.01 & \# & 0.02 & 0.03 \\
\hline Other religious & 0.10 & 0.01 & 0.06 & 0.13 & 0.01 & 0.32 & 0.03 \\
\hline Conservative Christian & 0.14 & 0.01 & 0.03 & 0.08 & 0.01 & 0.22 & 0.02 \\
\hline Other affiliated & 0.04 & \# & 0.03 & 0.08 & \# & 0.13 & 0.01 \\
\hline Unaffiliated & 0.19 & 0.01 & 0.14 & 0.36 & 0.02 & 0.79 & 0.07 \\
\hline Nonsectarian & 0.02 & \# & 0.04 & 0.15 & 0.01 & 0.15 & 0.03 \\
\hline Regular & 0.03 & \# & 0.05 & 0.22 & 0.01 & 0.21 & 0.04 \\
\hline Special emphasis & 0.02 & \# & 0.06 & 0.06 & 0.02 & 0.09 & 0.02 \\
\hline Special education & 0.06 & 0.01 & 0.01 & 0.08 & $\#$ & 0.14 & 0.03 \\
\hline \multicolumn{8}{|l|}{ School level } \\
\hline Elementary & 0.06 & \# & 0.02 & 0.04 & \# & 0.12 & 0.01 \\
\hline Secondary & 0.01 & 0.01 & 0.06 & 0.23 & 0.01 & 0.27 & 0.03 \\
\hline Combined & 0.09 & 0.01 & 0.06 & 0.10 & 0.01 & 0.29 & 0.04 \\
\hline \multicolumn{8}{|l|}{ Program emphasis } \\
\hline \multicolumn{8}{|l|}{ Regular elementary/ } \\
\hline secondary & 0.06 & \# & 0.03 & 0.06 & \# & 0.15 & 0.02 \\
\hline Montessori & 0.04 & \# & 0.11 & 0.03 & 0.02 & 0.15 & 0.04 \\
\hline Special program emphasis & 0.02 & 0.02 & 0.04 & 0.02 & \# & 0.05 & 0.01 \\
\hline Special education & 0.08 & 0.01 & 0.01 & 0.12 & \# & 0.19 & 0.03 \\
\hline Vocational/technical & $\dagger$ & $\dagger$ & $\dagger$ & $\dagger$ & $t$ & $\dagger$ & $t$ \\
\hline Alternative & 0.02 & \# & 0.01 & 0.18 & 0.06 & 0.14 & 0.01 \\
\hline Early childhood & 0.14 & 0.01 & 0.11 & 0.49 & 0.03 & 0.39 & 0.07 \\
\hline \multicolumn{8}{|l|}{ Size (number of students) } \\
\hline Less than 50 & 0.38 & 0.02 & 0.14 & 0.38 & 0.02 & 0.85 & 0.11 \\
\hline $50-149$ & 0.14 & 0.01 & 0.07 & 0.15 & 0.02 & 0.42 & 0.03 \\
\hline $150-299$ & 0.02 & \# & 0.02 & 0.11 & \# & 0.10 & 0.01 \\
\hline $300-499$ & 0.03 & \# & 0.02 & 0.04 & \# & 0.09 & 0.01 \\
\hline $500-749$ & 0.09 & 0.01 & 0.06 & 0.09 & \# & 0.11 & 0.03 \\
\hline 750 or more & 0.19 & 0.01 & 0.13 & 0.14 & 0.02 & 0.58 & 0.09 \\
\hline \multicolumn{8}{|l|}{ Region } \\
\hline Northeast & 0.13 & \# & 0.08 & 0.16 & \# & 0.40 & 0.03 \\
\hline Midwest & 0.12 & 0.01 & 0.05 & 0.13 & 0.01 & 0.26 & 0.03 \\
\hline South & 0.05 & \# & 0.01 & 0.09 & $\#$ & 0.09 & \# \\
\hline West & 0.01 & $\#$ & 0.01 & $\#$ & \# & 0.01 & \# \\
\hline \multicolumn{8}{|l|}{ Urbanicity type } \\
\hline City & 0.02 & \# & 0.03 & 0.10 & \# & 0.14 & 0.02 \\
\hline Suburban & 0.02 & \# & 0.02 & 0.03 & \# & 0.07 & 0.01 \\
\hline Town & 0.06 & 0.03 & 0.02 & 0.03 & 0.01 & 0.15 & 0.02 \\
\hline Rural & 0.22 & 0.04 & 0.12 & 0.16 & 0.02 & 0.58 & 0.07 \\
\hline
\end{tabular}

$\dagger$ Not applicable.

\# Rounds to zero.

SOURCE: U.S. Department of Education, National Center for Education Statistics, Private School Universe Survey (PSS), 2011-2012. 
Table C-10. Standard errors for percentage male enrollment in private schools and percentage distribution of private schools, by coeducational category and selected school characteristics: United States, 2011-12

\begin{tabular}{|c|c|c|c|c|}
\hline \multirow{2}{*}{$\begin{array}{l}\text { School } \\
\text { characteristic }\end{array}$} & \multirow{2}{*}{$\begin{array}{r}\text { Percent } \\
\text { male enrollment }\end{array}$} & \multicolumn{3}{|c|}{ Coeducational category } \\
\hline & & Coed schools & All-girls schools & All-boys schools \\
\hline Total & \# & 0.08 & 0.04 & 0.04 \\
\hline \multicolumn{5}{|l|}{ Private school typology } \\
\hline Catholic & \# & 0.03 & 0.02 & 0.01 \\
\hline Parochial & \# & 0.01 & \# & \\
\hline Diocesan & \# & 0.02 & 0.01 & 0.01 \\
\hline Private & \# & 0.54 & 0.29 & 0.25 \\
\hline Other religious & \# & 0.15 & 0.07 & 0.09 \\
\hline Conservative Christian & \# & 0.02 & $\dagger$ & 0.01 \\
\hline Other affiliated & \# & 0.20 & 0.20 & 0.02 \\
\hline Unaffiliated & \# & 0.28 & 0.09 & 0.20 \\
\hline Nonsectarian & \# & 0.03 & 0.01 & 0.02 \\
\hline Regular & \# & 0.04 & 0.02 & 0.02 \\
\hline Special emphasis & \# & 0.02 & \# & 0.02 \\
\hline Special education & \# & 0.10 & 0.02 & 0.09 \\
\hline \multicolumn{5}{|l|}{ School level } \\
\hline Elementary & \# & 0.02 & 0.01 & 0.01 \\
\hline Secondary & \# & 0.30 & 0.14 & 0.22 \\
\hline Combined & \# & 0.11 & 0.08 & 0.05 \\
\hline \multicolumn{5}{|l|}{ Program emphasis } \\
\hline \multicolumn{5}{|l|}{ Regular elementary/ } \\
\hline secondary & \# & 0.12 & 0.06 & 0.07 \\
\hline Montessori & \# & \# & $\dagger$ & \\
\hline Special program emphasis & \# & 0.05 & $\dagger$ & 0.03 \\
\hline Special education & \# & 0.11 & 0.02 & 0.09 \\
\hline Vocational/technical & $\dagger$ & $t$ & $\dagger$ & \\
\hline Alternative & \# & 0.03 & 0.01 & 0.02 \\
\hline Early childhood & \# & \# & $\dagger$ & 0.00 \\
\hline \multicolumn{5}{|l|}{ Size (number of students) } \\
\hline Less than 50 & \# & 0.10 & 0.03 & 0.08 \\
\hline $50-149$ & \# & 0.05 & 0.02 & 0.03 \\
\hline $150-299$ & \# & 0.11 & 0.11 & 0.01 \\
\hline $300-499$ & $\#$ & 0.02 & 0.01 & 0.01 \\
\hline $500-749$ & \# & 0.36 & 0.24 & 0.12 \\
\hline 750 or more & \# & 0.27 & 0.08 & 0.20 \\
\hline \multicolumn{5}{|l|}{ Region } \\
\hline Northeast & \# & 0.15 & 0.10 & 0.09 \\
\hline Midwest & \# & 0.14 & 0.07 & 0.08 \\
\hline South & \# & 0.02 & 0.01 & 0.01 \\
\hline West & \# & 0.02 & $\#$ & 0.02 \\
\hline \multicolumn{5}{|l|}{ Urbanicity type } \\
\hline City & \# & 0.07 & 0.06 & 0.02 \\
\hline Suburban & \# & 0.06 & 0.01 & 0.05 \\
\hline Town & \# & 0.03 & $\dagger$ & 0.02 \\
\hline Rural & \# & 0.19 & 0.05 & 0.14 \\
\hline
\end{tabular}

† Not applicable

\# Rounds to zero.

SOURCE: U.S. Department of Education, National Center for Education Statistics, Private School Universe Survey (PSS), 2011-2012. 
Table C-11. Standard errors for number and percentage distribution of private school teachers (headcount), by work status and selected characteristics: United States, 2011-12

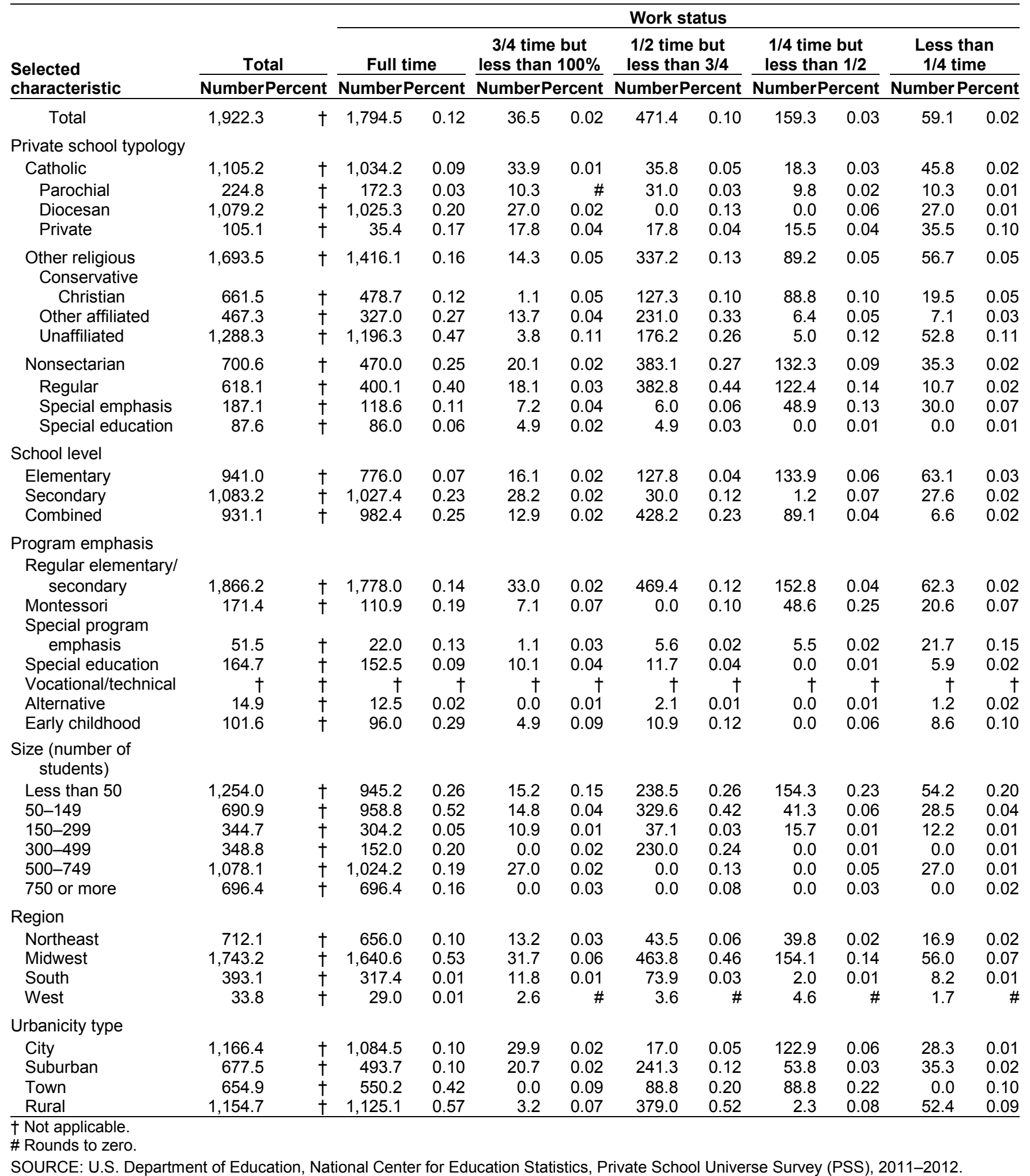


Table C-12. Standard errors for pupil/teacher ratio of private schools, by school level and selected characteristics: United States, 2011-12

\begin{tabular}{|c|c|c|c|c|}
\hline \multirow{2}{*}{$\begin{array}{l}\text { Selected } \\
\text { characteristic }\end{array}$} & \multirow[b]{2}{*}{ Total } & \multicolumn{3}{|c|}{ School level } \\
\hline & & Elementary & Secondary & Combined \\
\hline Total & 0.03 & 0.03 & 0.04 & 0.08 \\
\hline \multicolumn{5}{|l|}{ Private school typology } \\
\hline Catholic & 0.01 & 0.01 & \# & 0.01 \\
\hline Parochial & 0.01 & 0.01 & 0.00 & 0.06 \\
\hline Diocesan & 0.01 & \# & 0.04 & 0.02 \\
\hline Private & 0.02 & 0.10 & 0.00 & 0.00 \\
\hline Other religious & 0.08 & 0.07 & 0.03 & 0.13 \\
\hline Conservative Christian & 0.06 & 0.16 & 0.00 & 0.06 \\
\hline Other affiliated & 0.04 & 0.02 & 0.00 & 0.07 \\
\hline Unaffiliated & 0.21 & 0.14 & 0.07 & 0.41 \\
\hline Nonsectarian & 0.02 & 0.03 & 0.09 & 0.02 \\
\hline Regular & 0.03 & 0.07 & 0.14 & 0.03 \\
\hline Special emphasis & 0.01 & 0.01 & 0.04 & 0.01 \\
\hline Special education & 0.02 & 0.10 & 0.00 & 0.02 \\
\hline \multicolumn{5}{|l|}{ Program emphasis } \\
\hline \multicolumn{5}{|l|}{ Regular elementary/ } \\
\hline secondary & 0.04 & 0.03 & 0.03 & 0.09 \\
\hline Montessori & 0.01 & 0.01 & $\dagger$ & 0.00 \\
\hline Special program emphasis & \# & 0.01 & 0.00 & 0.01 \\
\hline Special education & 0.02 & 0.09 & 0.01 & 0.03 \\
\hline Vocational/technical & $\dagger$ & $\dagger$ & $\dagger$ & $\dagger$ \\
\hline Alternative & \# & 0.01 & 0.01 & 0.00 \\
\hline Early childhood & 0.04 & 0.04 & $\dagger$ & 0.00 \\
\hline \multicolumn{5}{|l|}{ Size (number of students) } \\
\hline Less than 50 & 0.06 & 0.10 & 0.01 & 0.05 \\
\hline $50-149$ & 0.02 & 0.05 & 0.01 & 0.03 \\
\hline $150-299$ & 0.02 & 0.01 & 0.10 & 0.04 \\
\hline $300-499$ & 0.02 & 0.01 & 0.00 & 0.02 \\
\hline $500-749$ & 0.02 & 0.00 & 0.02 & 0.00 \\
\hline 750 or more & 0.15 & 0.00 & 0.00 & 0.25 \\
\hline \multicolumn{5}{|l|}{ Region } \\
\hline Northeast & 0.11 & 0.04 & 0.01 & 0.31 \\
\hline Midwest & 0.07 & 0.08 & 0.04 & 0.13 \\
\hline South & 0.02 & 0.04 & 0.06 & \# \\
\hline West & \# & \# & \# & \# \\
\hline \multicolumn{5}{|l|}{ Urbanicity type } \\
\hline City & 0.02 & 0.02 & 0.04 & 0.02 \\
\hline Suburban & 0.02 & 0.03 & 0.02 & 0.02 \\
\hline Town & 0.12 & \# & 0.00 & 0.24 \\
\hline Rural & 0.19 & 0.20 & \# & 0.32 \\
\hline
\end{tabular}

† Not applicable.

\# Rounds to zero.

SOURCE: U.S. Department of Education, National Center for Education Statistics, Private School Universe Survey (PSS), 2011-2012. 
Table C-13. Standard errors for percentage of private schools with 12th-graders, number of graduates, graduation rate, and percentage of graduates who attended 4-year colleges, by selected selected characteristics: United States, 2010-11

\begin{tabular}{|c|c|c|c|c|}
\hline $\begin{array}{l}\text { Selected } \\
\text { characteristic }\end{array}$ & $\begin{array}{r}\text { Percent of schools } \\
\text { with 12th-graders } \\
\text { in 2010-11 }\end{array}$ & $\begin{array}{r}\text { Number of } \\
2010-11 \\
\text { graduates }\end{array}$ & $\begin{array}{r}2010-11 \\
\text { graduation } \\
\text { rate }\end{array}$ & $\begin{array}{r}\text { Percent of 2010-11 } \\
\text { graduates } \\
\text { who attended } \\
\text { 4-year colleges } \\
\text { by fall } 2011\end{array}$ \\
\hline Total & 0.33 & $3,479.2$ & 0.09 & 0.74 \\
\hline Private school typology & & & & \\
\hline $\begin{array}{l}\text { Catholic } \\
\text { Parochial } \\
\text { Diocesan } \\
\text { Private }\end{array}$ & $\begin{array}{l}0.36 \\
0.04 \\
0.76 \\
0.92\end{array}$ & $\begin{array}{r}3,153.5 \\
0.0 \\
3,153.5 \\
0.0\end{array}$ & $\begin{array}{l}0.21 \\
0.00 \\
0.46 \\
0.00\end{array}$ & $\begin{array}{l}0.43 \\
0.00 \\
0.99 \\
0.00\end{array}$ \\
\hline $\begin{array}{l}\text { Other religious } \\
\text { Conservative Christian } \\
\text { Other affiliated } \\
\text { Unaffiliated }\end{array}$ & $\begin{array}{l}0.78 \\
1.37 \\
0.23 \\
1.58\end{array}$ & $\begin{array}{r}1,441.9 \\
384.5 \\
90.0 \\
1,465.3\end{array}$ & $\begin{array}{l}0.02 \\
0.02 \\
0.01 \\
0.11\end{array}$ & $\begin{array}{l}1.39 \\
2.48 \\
0.51 \\
1.50\end{array}$ \\
\hline $\begin{array}{l}\text { Nonsectarian } \\
\text { Regular } \\
\text { Special emphasis } \\
\text { Special education }\end{array}$ & $\begin{array}{l}0.38 \\
0.73 \\
0.16 \\
0.73\end{array}$ & $\begin{array}{r}577.8 \\
572.5 \\
78.0 \\
1.0\end{array}$ & $\begin{array}{l}0.04 \\
0.02 \\
0.11 \\
0.02\end{array}$ & $\begin{array}{l}1.06 \\
2.86 \\
0.56 \\
0.03\end{array}$ \\
\hline $\begin{array}{l}\text { School level } \\
\text { Elementary } \\
\text { Secondary } \\
\text { Combined }\end{array}$ & $\begin{array}{r}\dagger \\
0.21 \\
1.31\end{array}$ & $\begin{array}{r}t \\
3,193.1 \\
1,379.1\end{array}$ & $\begin{array}{r}\dagger \\
0.16 \\
0.02\end{array}$ & $\begin{array}{r}\dagger \\
0.30 \\
1.01\end{array}$ \\
\hline $\begin{array}{l}\text { Program emphasis } \\
\text { Regular elementary/ } \\
\text { secondary } \\
\text { Montessori } \\
\text { Special program emphasis } \\
\text { Special education } \\
\text { Vocational/technical } \\
\text { Alternative } \\
\text { Early childhood }\end{array}$ & $\begin{array}{r}0.54 \\
0.19 \\
0.37 \\
0.68 \\
\dagger \\
0.11 \\
\dagger\end{array}$ & $\begin{array}{r}3,482.4 \\
78.0 \\
0.0 \\
22.3 \\
\dagger \\
1.2 \\
\dagger\end{array}$ & $\begin{array}{r}0.10 \\
1.45 \\
0.00 \\
0.07 \\
\dagger \\
0.08 \\
\dagger\end{array}$ & $\begin{array}{r}0.94 \\
2.22 \\
0.00 \\
0.26 \\
\dagger \\
0.13 \\
\dagger\end{array}$ \\
\hline $\begin{array}{l}\text { Size (number of students) } \\
\text { Less than } 50 \\
50-149 \\
150-299 \\
300-499 \\
500-749 \\
750 \text { or more }\end{array}$ & $\begin{array}{l}0.48 \\
0.34 \\
0.14 \\
0.14 \\
0.79 \\
0.13\end{array}$ & $\begin{array}{r}124.0 \\
115.4 \\
495.2 \\
45.9 \\
3,153.5 \\
1,462.5\end{array}$ & $\begin{array}{r}0.18 \\
0.04 \\
0.02 \\
\# \\
0.44 \\
\#\end{array}$ & $\begin{array}{r}2.48 \\
0.19 \\
0.40 \\
0.04 \\
0.04 \\
\#\end{array}$ \\
\hline $\begin{array}{l}\text { Region } \\
\text { Northeast } \\
\text { Midwest } \\
\text { South } \\
\text { West }\end{array}$ & $\begin{array}{l}0.27 \\
1.03 \\
0.30 \\
0.03\end{array}$ & $\begin{array}{r}1,374.1 \\
3,158.5 \\
489.6 \\
29.1\end{array}$ & $\begin{array}{l}0.03 \\
0.40 \\
0.01 \\
0.01\end{array}$ & $\begin{array}{l}0.63 \\
3.44 \\
0.14 \\
0.10\end{array}$ \\
\hline $\begin{array}{l}\text { Urbanicity type } \\
\text { City } \\
\text { Suburban } \\
\text { Town } \\
\text { Rural }\end{array}$ & $\begin{array}{l}0.24 \\
0.19 \\
2.23 \\
2.06\end{array}$ & $\begin{array}{r}3,193.2 \\
90.1 \\
88.8 \\
1,465.3\end{array}$ & $\begin{array}{l}0.19 \\
0.01 \\
0.01 \\
0.07\end{array}$ & $\begin{array}{l}0.31 \\
0.20 \\
6.27 \\
0.95\end{array}$ \\
\hline
\end{tabular}


Table C-14. Standard errors for number of private schools, students, and teachers (headcount), by school membership in private school associations: United States, 2011-12

\begin{tabular}{|c|c|c|c|}
\hline Association & Schools & Students & Teachers \\
\hline Total & 542.4 & $25,138.6$ & $1,922.3$ \\
\hline None & 452.2 & $19,837.2$ & $1,287.1$ \\
\hline \multicolumn{4}{|l|}{ Religious } \\
\hline Accelerated Christian Education & 126.9 & $4,606.5$ & 658.8 \\
\hline American Association of Christian Schools & 9.7 & 564.7 & 58.4 \\
\hline Association of Christian Schools International & 1.0 & 58.4 & 9.2 \\
\hline Association of Christian Teachers and Schools & 0.0 & 0.0 & 0.0 \\
\hline Association of Classical and Christian Schools & 1.5 & 400.5 & 43.4 \\
\hline Christian Schools International & 0.0 & 0.0 & 0.0 \\
\hline Evangelical Lutheran Education Association & 1.0 & 16.0 & 3.0 \\
\hline Friends Council on Education & 0.0 & 0.0 & 0.0 \\
\hline General Conference of the Seventh-Day Adventist Church & 0.0 & 0.0 & 0.0 \\
\hline Islamic School League of America & 0.0 & 0.0 & 0.0 \\
\hline Jesuit Secondary Education Association & 0.0 & 0.0 & 0.0 \\
\hline National Association of Episcopal Schools & 0.0 & 0.0 & 0.0 \\
\hline National Catholic Educational Association & 29.0 & $14,503.5$ & $1,088.3$ \\
\hline National Christian School Association & 0.0 & 0.0 & 0.0 \\
\hline National Society of Hebrew Day Schools & 4.6 & $1,666.2$ & 321.3 \\
\hline Oral Roberts University Educational Fellowship & 0.0 & 0.0 & 0.0 \\
\hline The Jewish Community Day School Network & 6.4 & $1,220.2$ & 287.5 \\
\hline Solomon Schechter Day School Association & 0.0 & 0.0 & 0.0 \\
\hline Southern Baptist Association of Christian Schools & 0.0 & 0.0 & 0.0 \\
\hline Other religious school associations & 75.9 & $1,642.0$ & 418.5 \\
\hline \multicolumn{4}{|l|}{ Special emphasis } \\
\hline American Montessori Society & 11.3 & 152.0 & 29.6 \\
\hline Association Montessori International & 4.1 & 69.0 & 12.0 \\
\hline Other Montessori associations & 4.7 & 408.5 & 50.5 \\
\hline Association of Military Colleges and Schools & 0.0 & 0.0 & 0.0 \\
\hline Association of Waldorf Schools of North America & 0.0 & 0.0 & 0.0 \\
\hline National Association of Private Special Education Centers & 0.0 & 0.0 & 0.0 \\
\hline Other associations for exceptional children & 1.2 & 46.1 & 5.9 \\
\hline European Council for International Schools & $\dagger$ & $\dagger$ & $\dagger$ \\
\hline National Association for the Education of Young Children & 15.5 & 190.7 & 15.5 \\
\hline National Association of Laboratory Schools & 0.0 & 0.0 & 0.0 \\
\hline National Coalition of Girls' Schools & 0.0 & 0.0 & 0.0 \\
\hline Other special emphasis school associations & 0.0 & 0.0 & 0.0 \\
\hline \multicolumn{4}{|l|}{ Other school associations or organizations } \\
\hline Alternative School Network & 0.0 & 0.0 & 0.0 \\
\hline National Association of Independent Schools & 0.0 & 0.0 & 0.0 \\
\hline State or regional independent school association & 5.5 & 116.4 & 25.6 \\
\hline National Coalition of Alternative Community Schools & 0.0 & 0.0 & 0.0 \\
\hline National Independent Private School Association & 0.0 & 0.0 & 0.0 \\
\hline The Association of Boarding Schools & 0.0 & 0.0 & 0.0 \\
\hline Other school associations & 33.1 & $3,357.9$ & 333.3 \\
\hline
\end{tabular}

† Not applicable.

SOURCE: U.S. Department of Education, National Center for Education Statistics, Private School Universe Survey (PSS), 2011-2012. 
Table C-15. Standard error for number of private schools, students, full-time equivalent (FTE) teachers, and 2010-11 high school graduates, by state: United States, 2011-12

\begin{tabular}{|c|c|c|c|c|}
\hline State & Schools & Students & $\begin{array}{r}\text { FTE } \\
\text { teachers }\end{array}$ & $\begin{array}{r}\text { High school } \\
\text { graduates 2010-11 }\end{array}$ \\
\hline United States & 542.4 & $25,138.6$ & $1,836.0$ & $3,479.2$ \\
\hline $\begin{array}{l}\text { Alabama } \\
\text { Alaska } \\
\text { Arizona } \\
\text { Arkansas } \\
\text { California }\end{array}$ & $\begin{array}{r}1.3 \\
0.0 \\
4.7 \\
73.2 \\
1.8\end{array}$ & $\begin{array}{r}29.0 \\
0.0 \\
132.1 \\
1,171.7 \\
83.7\end{array}$ & $\begin{array}{r}0.8 \\
0.0 \\
16.2 \\
336.9 \\
12.1\end{array}$ & $\begin{array}{l}0.0 \\
0.0 \\
0.0 \\
0.0 \\
1.0\end{array}$ \\
\hline $\begin{array}{l}\text { Colorado } \\
\text { Connecticut } \\
\text { Delaware } \\
\text { District of Columbia } \\
\text { Florida }\end{array}$ & $\begin{array}{r}2.0 \\
17.8 \\
0.0 \\
0.0 \\
2.3\end{array}$ & $\begin{array}{r}55.7 \\
142.2 \\
0.0 \\
0.0 \\
140.4\end{array}$ & $\begin{array}{r}4.1 \\
67.5 \\
0.0 \\
0.0 \\
36.5\end{array}$ & $\begin{array}{l}0.0 \\
0.0 \\
0.0 \\
0.0 \\
0.0\end{array}$ \\
\hline $\begin{array}{l}\text { Georgia } \\
\text { Hawaii } \\
\text { Idaho } \\
\text { Illinois } \\
\text { Indiana }\end{array}$ & $\begin{array}{r}0.0 \\
0.0 \\
4.2 \\
53.4 \\
198.2\end{array}$ & $\begin{array}{r}0.0 \\
0.0 \\
186.5 \\
1,261.2 \\
11,871.0\end{array}$ & $\begin{array}{r}0.0 \\
0.0 \\
17.3 \\
221.0 \\
994.9\end{array}$ & $\begin{array}{r}0.0 \\
0.0 \\
3.8 \\
49.4 \\
374.1\end{array}$ \\
\hline $\begin{array}{l}\text { lowa } \\
\text { Kansas } \\
\text { Kentucky } \\
\text { Louisiana } \\
\text { Maine }\end{array}$ & $\begin{array}{r}50.5 \\
193.2 \\
1.0 \\
2.0 \\
0.0\end{array}$ & $\begin{array}{r}14,664.7 \\
1,639.8 \\
12.0 \\
38.7 \\
0.0\end{array}$ & $\begin{array}{r}1,078.2 \\
445.9 \\
1.0 \\
4.6 \\
0.0\end{array}$ & $\begin{array}{l}\dagger \\
0.0 \\
0.0 \\
0.0 \\
0.0\end{array}$ \\
\hline $\begin{array}{l}\text { Maryland } \\
\text { Massachusetts } \\
\text { Michigan } \\
\text { Minnesota } \\
\text { Mississippi }\end{array}$ & $\begin{array}{r}8.2 \\
43.6 \\
7.0 \\
0.0 \\
0.0\end{array}$ & $\begin{array}{r}389.7 \\
985.3 \\
216.4 \\
0.0 \\
0.0\end{array}$ & $\begin{array}{r}42.2 \\
322.8 \\
51.7 \\
0.0 \\
0.0\end{array}$ & $\begin{array}{l}0.0 \\
0.0 \\
0.0 \\
0.0 \\
0.0\end{array}$ \\
\hline $\begin{array}{l}\text { Missouri } \\
\text { Montana } \\
\text { Nebraska } \\
\text { Nevada } \\
\text { New Hampshire }\end{array}$ & $\begin{array}{r}467.7 \\
0.0 \\
0.0 \\
0.0 \\
0.0\end{array}$ & $\begin{array}{r}8,052.4 \\
0.0 \\
0.0 \\
0.0 \\
0.0\end{array}$ & $\begin{array}{r}950.7 \\
0.0 \\
0.0 \\
0.0 \\
0.0\end{array}$ & $\begin{array}{r}88.8 \\
0.0 \\
0.0 \\
0.0 \\
0.0\end{array}$ \\
\hline $\begin{array}{l}\text { New Jersey } \\
\text { New Mexico } \\
\text { New York } \\
\text { North Carolina } \\
\text { North Dakota }\end{array}$ & $\begin{array}{r}18.3 \\
1.0 \\
31.0 \\
0.0 \\
0.0\end{array}$ & $\begin{array}{r}688.0 \\
10.3 \\
18,737.9 \\
0.0 \\
0.0\end{array}$ & $\begin{array}{r}208.3 \\
1.9 \\
821.2 \\
0.0 \\
0.0\end{array}$ & $\begin{array}{r}70.7 \\
0.0 \\
1,462.5 \\
0.0 \\
\dagger\end{array}$ \\
\hline $\begin{array}{l}\text { Ohio } \\
\text { Oklahoma } \\
\text { Oregon } \\
\text { Pennsylvania } \\
\text { Rhode Island }\end{array}$ & $\begin{array}{r}59.2 \\
9.6 \\
0.0 \\
60.1 \\
0.0\end{array}$ & $\begin{array}{r}2,620.0 \\
698.2 \\
0.0 \\
3,510.8 \\
0.0\end{array}$ & $\begin{array}{r}395.7 \\
71.6 \\
0.0 \\
467.5 \\
0.0\end{array}$ & $\begin{array}{r}123.9 \\
17.1 \\
0.0 \\
90.2 \\
0.0\end{array}$ \\
\hline $\begin{array}{l}\text { South Carolina } \\
\text { South Dakota } \\
\text { Tennessee } \\
\text { Texas } \\
\text { Utah }\end{array}$ & $\begin{array}{r}0.0 \\
0.0 \\
1.1 \\
17.9 \\
2.2\end{array}$ & $\begin{array}{r}0.0 \\
0.0 \\
13.0 \\
1,852.5 \\
55.4\end{array}$ & $\begin{array}{r}0.0 \\
0.0 \\
2.2 \\
101.3 \\
5.9\end{array}$ & $\begin{array}{r}0.0 \\
0.0 \\
0.0 \\
489.3 \\
1.2\end{array}$ \\
\hline $\begin{array}{l}\text { Vermont } \\
\text { Virginia } \\
\text { Washington } \\
\text { West Virginia } \\
\text { Wisconsin } \\
\text { Wyoming }\end{array}$ & $\begin{array}{l}0.0 \\
2.1 \\
5.4 \\
1.0 \\
0.0 \\
0.0\end{array}$ & $\begin{array}{r}0.0 \\
81.9 \\
232.5 \\
1.0 \\
0.0 \\
0.0 \\
\end{array}$ & $\begin{array}{r}0.0 \\
11.0 \\
21.5 \\
1.0 \\
0.0 \\
0.0\end{array}$ & $\begin{array}{r}0.0 \\
1.1 \\
30.0 \\
1.0 \\
0.0 \\
+\end{array}$ \\
\hline
\end{tabular}

† Not applicable.

SOURCE: U.S. Department of Education, National Center for Education Statistics, Private School Universe Survey (PSS), $2011-2012$. 